Research Article

\title{
Nonlinear Buckling Analysis of Thin-Walled Box Beams considering Shear Lag
}

\author{
Minyao Tan $(\mathbb{D})$ and Wenming Cheng $(\mathbb{D})$ \\ Department of Mechanical Engineering, South West Jiaotong University, Chengdu, China \\ Correspondence should be addressed to Minyao Tan; minyaotan@163.com
}

Received 29 April 2020; Revised 23 June 2020; Accepted 30 June 2020; Published 28 August 2020

Academic Editor: Roberto G. Citarella

Copyright (C) 2020 Minyao Tan and Wenming Cheng. This is an open access article distributed under the Creative Commons Attribution License, which permits unrestricted use, distribution, and reproduction in any medium, provided the original work is properly cited.

\begin{abstract}
In this work, a general geometric nonlinear model of straight thin-walled box beams (STBBs) under combined eccentric and axial loads is established. In order to accurately reflect the behavior of STBB, the additional shear lag warping is added to enrich the displacement field. It is necessary to define the section shape function to describe the local section deformation. Therefore, extension, bending, torsion, distortion, and shear lag effects are expressed by the generalized coordinate method. Based on the stability of transverse unconstrained box beam theory, meaningful higher-order solutions can be obtained by defining a set of coupled deformation modes. The equilibrium equation is discretized by the Galerkin method, and the Newton-Raphson incremental method is used to derive and solve the nonlinear governing equations. On this basis, the analytical expression of stiffness matrix is established. For solving the stability problem, the effectiveness of the proposed method is verified by comparing the calculation results of shell element (Ansys) with other theories. Numerical examples even show that the proposed method can not only get the influence of shear lag but also obtain the variation of lateral buckling of the beam model.
\end{abstract}

\section{Introduction}

As a kind of important load-bearing structural member, performance and design are a very significant aspect of thinwalled elements because they minimize the weight and cost of the structure. The classical Vlasov beam theory, St. Venant torsion theory, and the thin-walled beam theory put forward several calculation formulas including transverse deformation and displacement, according to the research of different open and closed cross sections [1]. On the account of the kinematics hypothesis that the shear strain was ignored in the central line of the cross section, classical formulas about distortion theory were proposed on the basis of Vlasov theory. Correia et al. discussed the buckling behavior and strength of glass fiber reinforced polymer (GFRP) under uniform compression and conducted experimental, numerical, and analytical studies, focusing on local buckling [2]. Based on the theory of small strain and medium rotations, Ascione et al. established the kinematics model of composite thin-wall beam. The effects of geometrical imperfections and shear deformation on the members were fully considered, and the prebuckling behavior, the global buckling, and local buckling analyses were carried out [3-6].

In general, the deflection of box beam under self-weight and load may be quite different when the shear lag effect is ignored in the traditional beam theory. Reissner was the first one to use the variational energy method to analyze the biaxial symmetric rectangular box girder and the quadratic parabola as the displacement function of shear lag buckling [7]. Later, many scholars adopted quadratic, cubic, or even quadrilateral parabolas as the displacement function of shear lag on the basis of Reissner's theory [8-12]. Based on these methods, Luo and $\mathrm{Wu}$ selected three independent generalized displacements of top, bottom, and side cantilever plates, respectively, to represent different shear lag warping displacements between box girder plates with trapezoidal sections [8]. According to the energy method, Lin and Zhao described the nonuniform longitudinal displacement of flanges by using the higher-order polynomial of the infinite term and further quantified the shear lag effect of 
thin-walled bending members such as T-beams and box beams [13]. In order to solve the problem of plastic deformation of steel box girder in the limit condition, Lin and Zhao proposed a method for modeling the behavior of inelastic shear lag [14]. Lertsima et al. used shell element modeling for the entire simply supported box girder and studied the stress concentration problem at the flange under the influence of shear lag by using the three-dimensional finite element method [15]. In the same way, Sa-nguanmanasak treated the influence of finite element mesh on shear lag in detail by using the method of multi-degree-offreedom extrapolation and derived the empirical formula to calculate the stress concentration coefficient including the shear lag effect [16]. Then, Zhang put forward generalized formulas, which included the geometric properties of sections and generalized internal forces concerning shear lag moments and bending moments [17]. Chen et al. obtained a closed solution of shear lag based on the variational method of energy and carried out harmonic analysis on the closed beam [18]. In addition, Li improved the PM-DP analysis method, which can be used to correct the defects of shear lag warping deformation of the top cantilever flange [19].

The good structural performance of thin-walled box girder has always been the focus of research by many scholars. Considering the coupling of bending, torsion, shear deformation, and warping shear stress, Wang proposed a new type of nonshearing locking thin-wall section space beam element [20]. In view of distortion, a new one-dimensional theory was proposed by Law and Kim, in which the static and dynamic studies on thin-walled closed beams with general cross sections were carried out [21]. The research of Xu and Ye showed that the single box beam had no obvious pure distortion, and the influence of distortion shear stress on shear deformation was analyzed [22]. Razaqpur and Li developed a new finite element model of curved thin-walled multicompartment box girder by combining the generalized Vlasov theory with the FEA technique, which could simulate the extension, bending, torsion, torsional warping, distortion, distortional warping, and shear lag effects of thin-walled box beam [23]. In order to calculate the section deformation, Choi and Jang adopted the beam frame method of two-dimensional Euler beam for simulation and developed the FEA model of quadrilateral thin-walled beam with variable section using the high-order beam theory [24]. It was approximated using the Ritz-Galerkin method's independent descriptions of stress and displacement fields. According to the previous work, Tan further analyzed nonlinear lateral buckling of thin-walled box beam's webs with the unequal thickness by considering the coupling of the bending, torsion, and distortion [25].

The deformation of box girder section under eccentric load and axial force is also studied. Genoese and Pietraszkiewiczestablished the linear kinematic equations for a thin-walled beam of heterogeneous isotropic material [26]. Opoka and Pietraszkiewicz showed the complete boundary value problem (BVP) for geometrical nonlinearity of thin shells, which was the stress action and bending of the reference surface of shells [27]. Coupling both material anisotropy and geometric nonlinearity, Vo presented a general geometric nonlinear model of arbitrary spatial composite thin-walled beams under different loading [28]. Miranda and Gutiérrez developed a new generalized beam theory (GBT), which modified the kinematics formulations of shearing deformation GBT put forward by Silvestre and Camotim in the early period [29]. Considering the effects of axial warping effect and material anisotropy on the surface shear strain in the shell, Zhang proposed an improved structural mechanics model of composite beams with single closed thin-walled section [30]. Meanwhile, Sahraei extended the shear deformation theory and FEA method with computational efficiency to the calculation of the transverse torsional buckling capacity of single symmetric I-beams under general load [31]. Moreover, CambroneroBarrientos identified a beam element with three nodes, each of which has five degrees of freedom, which exceeded six degrees of freedom of traditional three-dimensional beam [32]. And the influence of homogeneous and nonhomogeneous torsion, distortion, and shear lag had been considered in the normal stress distribution at the same time. Then, Meftah investigated the elastic lateral buckling distortional deformation of thinwalled box girder under the combined action of bending and axial loads [33].

In this paper, shear lag effect is considered as a separate mechanical property in the displacement deformation. So, the displacements of straight thin-walled box beams (STBBs) are divided into the sum of the partial displacements of six deformation fields. The main purpose of this work is to consider the influence of shear lag on nonlinear buckling performance of box girder under the combined action of an eccentric load and axial force.

\section{Theoretical Model for Buckling Analysis}

In this section, the main symbols and conventions of symbols are given, then the displacement field are described, and the kinematic equations are given on the basis of references $[12,21]$. Therefore, the stiffness matrix is finally obtained.

It is assumed that the displacement field is the sum of the modes of each displacement deformation. Here, since the shear forces $Q_{z}$ and $Q_{y}$ are unknown, it is essential to modify it through the orthogonal warping shear function $\omega_{\zeta z}$ and $\omega_{\zeta y}$ when the shear lag effect is analyzed.

2.1. Basic Assumptions and Considerations. The present assumptions about the structural model are as follows:

(1) The material of the model is linear elastic and homogeneous. The influence of the thickness of each wall on warping is ignored.

(2) The strain on the contour of each plane is zero, that is, the plane of STBB cannot expand in the tangential direction.

(3) The member buckles under the nonextensional mode, so the curvature and central longitudinal strain of the $x z$ plane remain zero during the instability process. This means that members are supposed to buckle under combined axial and bending loads. 
2.2. Deformation Modes. Figure 1 shows the global Cartesian coordinate system $(x, y, z)$, where the $x$-axis is the same as the longitudinal direction of STBB. $(s, n, x)$ is the local coordinate system. For each wall, $s$ passes through the whole cross section along the center line of each plate, and $n$ is the coordinate of the local normal line.

The generalized displacement theory of STBB is based on the assumption of deformation modes (in Figures 2 and 3) corresponding to the extension, bending, torsion, shear lag, and distortion. These modes can be separated into a group of coupled axial and transverse displacement fields, each of which can be decomposed into the functions of the plane and axial coordinates. In the following, it makes sense to note that the displacement modes including global and local modes are described in detail.

Extensional mode, which only produces constant displacement $u$ in the direction of length, is the simplest kind of displacement.

Two bending modes generate a displacement and a rotation, respectively. The rigid solid displacements ( $v$ and $w$ ) are in the plane of cross section and the rotations $\left(\theta_{y}\right.$ and $\left.\theta_{z}\right)$ are about the $y$ - and $z$-axes.

Shear lag deflections considering shear forces, the generalized displacement of shear lag deformations $\left(\zeta_{z}\right.$ and $\left.\zeta_{y}\right)$, and shear lag warping displacement functions $\left(\omega_{\zeta z}\right.$ and $\left.\omega_{\zeta y}\right)$ are introduced, which are proportional to shear forces $Q_{z}$ and $Q_{y}$, respectively. Therefore, the additional displacements caused by the shear lag are $u_{\zeta z}=-\omega_{\zeta z} \zeta_{z}$ and $u_{\zeta y}=-\omega_{\zeta y} \cdot \zeta_{z}$. In the analysis of shear lag effect of thin-walled box beam, reference [12] interprets the quadratic parabola theory as a reasonable model of warping displacement function mode, the specific form of which will be detailed in the following part.

Torsion mode (no transverse deformation occurs in the cross section) generates homogeneous and nonhomogeneous torsional warping $\omega_{a}$ and $\omega_{a t}$, which are related to the normal stress $\sigma_{x}=E \omega_{a} \cdot \mathrm{d}^{2} \theta_{x} / \mathrm{d} x^{2}$ and the shear stress $\tau_{s x}=G \cdot \partial \omega_{a t} / \partial s \cdot \mathrm{d}^{3} \theta_{x} / \mathrm{d} x^{3}$, respectively. Therefore, the displacement field contains a rigid solid rotation $\theta_{x}$ in regard to the shear center $\left(x_{0}, y_{0}\right)$ of the cross section and two longitudinal displacements-the first- and third-order derivatives of torsion rotation are multiplied by the two warping functions, respectively. Nonhomogeneous torsional warping $\omega_{a t}$ is caused by the shear stresses. For the calculation of longitudinal displacement, the influence of nonhomogeneous torsion on normal stresses can be ignored.

Distortion mode is the transverse deformation of the cross section. The difference between homogeneous and nonhomogeneous distortion is not discussed in traditional theories. For distortion mode, transverse displacements are $\left(\eta \cdot \chi, \psi_{s} \cdot \chi\right)$ in the plane of the cross section. And in terms of free warping or nonfree warping, homogeneous distortion and nonhomogeneous distortion can be obtained. Considering that the thickness of the beam girder compared with other dimensions is very small, the compression of the box wall along the direction of the wall thickness was neglected currently. Then, the longitudinal displacement at any point of the beam can be given by the homogeneous and nonhomogeneous distortion warping $\omega_{d}$ and $\omega_{d t}$ multiplied, respectively, by the first- and third-order derivatives of distortion rotation $\chi$.
In the analysis of deformation modes, the transverse and longitudinal displacements are introduced as

$$
\begin{aligned}
v(y, z, x)= & v_{0}-\left(z-z_{0}\right) \theta_{x}(x)+\eta(s, n) \chi(x), \\
w(y, z, x)= & w_{0}-\left(y-y_{0}\right) \theta_{x}(x)+\psi_{s}(s) \chi(x), \\
u(y, z, x)= & u_{0}-z \theta_{y}-y \theta_{z}-\omega_{\zeta_{z}} \zeta_{z}(x)-\omega_{\zeta_{y}} \zeta_{y}(x) \\
& +\omega_{a} \frac{\mathrm{d} \theta_{\mathrm{x}}(x)}{\mathrm{d} x}+\omega_{a t} \frac{\mathrm{d}^{3} \theta_{\mathrm{x}}(x)}{\mathrm{d} x^{3}}+\omega_{d} \frac{\mathrm{d} \chi(x)}{\mathrm{d} x} \\
& +\omega_{d t} \frac{\mathrm{d}^{3} \chi(x)}{\mathrm{d} x^{3}} .
\end{aligned}
$$

2.3. Structural Mechanisms. In order to illustrate the general method of obtaining deformation modes of STBB under combined loads, the geometric dimension of STBB is defined as follows: $b$ is the width; $h$ is the height; $L$ denotes the entire beam length; and $t_{i}$ denotes the thickness of each wall which is much smaller than other dimensions.

It is well known that the shear lag effect inevitably increases the deflection of the box girder. The shear lag deformation state is described by generalized displacement, that is, additional deflection caused by shear lag effect.

The shear lag warping displacement function is not a continuous function throughout the beam that directly affects the size and distribution of cross section stress. Therefore, many authors studied and proved these interpolation of functions [8-12]. The shear lag warping stress $\sigma_{\omega \zeta z}=-E \omega_{\zeta z} \omega_{\zeta z}^{\prime}(x)$ (without the influence of axial force) of the cross section of the thin-walled structure should meet the self-balancing condition, that is,

$$
\begin{gathered}
\int_{\mathrm{A}} \sigma_{\omega \zeta z} \mathrm{~d} A=0, \\
\int_{\mathrm{A}} \sigma_{\omega \zeta z} \mathrm{z} \mathrm{d} A=0 .
\end{gathered}
$$

When the thin-walled box beams are bent, the normal stress of the cross section must satisfy the axial equilibrium condition, which means $\int_{A} \omega_{\zeta z} \mathrm{~d} A=0$. After many comparisons, it is reasonable to select the quadratic parabola as the shear lag warping displacement function. In this paper, the quadratic parabola expression of the selected shear lag warping displacement function is

$$
\omega_{\zeta_{z}}^{i}(s)= \begin{cases}-\left(\frac{h}{2}-z_{0}\right)\left(1-\frac{4 s^{2}}{b^{2}}\right)+d, & i=1, \\ d, & i=2,4, \\ \left(\frac{h}{2}+z_{0}\right)\left(1-\frac{4 s^{2}}{b^{2}}\right)\left(\frac{h+2 z_{0}}{h-2 z_{0}}\right)+d, & i=3,\end{cases}
$$

$$
d=\frac{2\left(h / 2-z_{0}\right)}{3 A}\left[b t_{1}-b t_{3}\left(\frac{h+2 z_{0}}{h-2 z_{0}}\right)^{2}\right]
$$




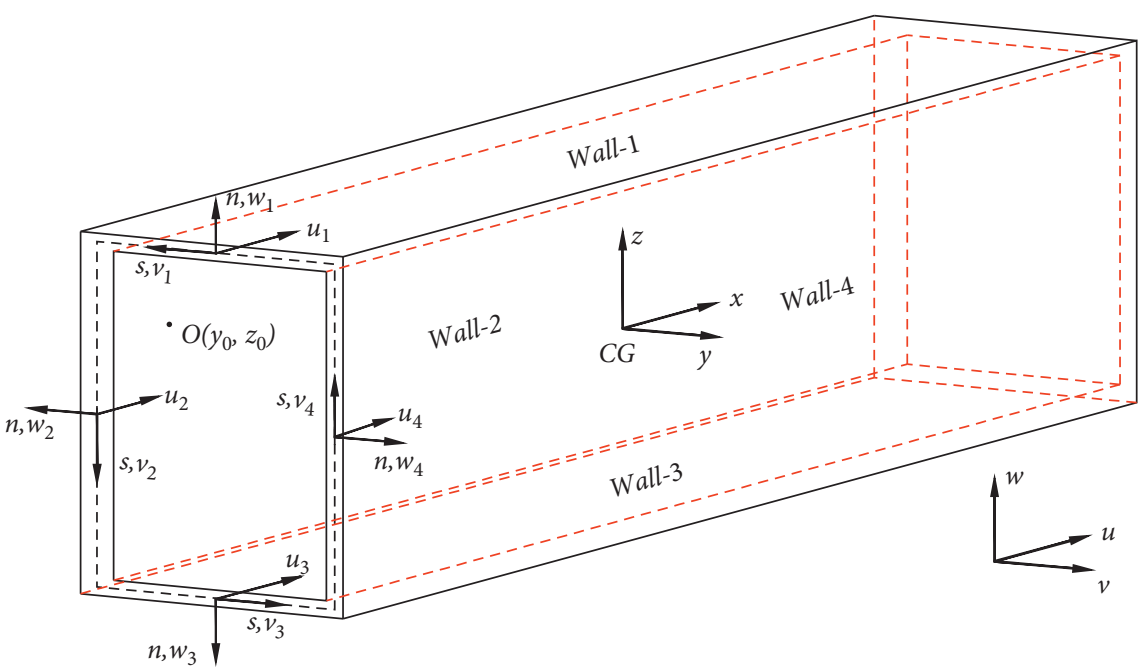

FIgURE 1: Cartesian coordinate system and the local coordinate of each plane.

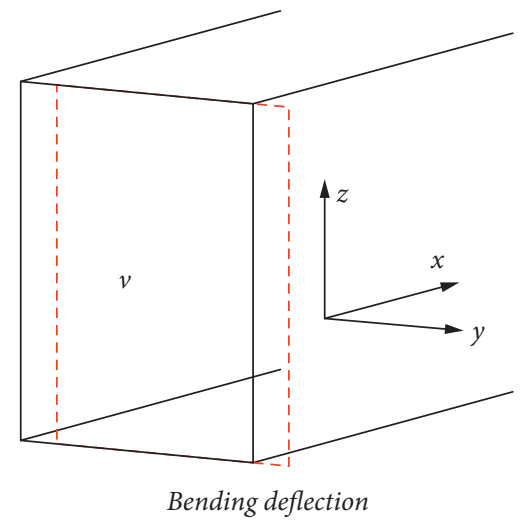

(a)

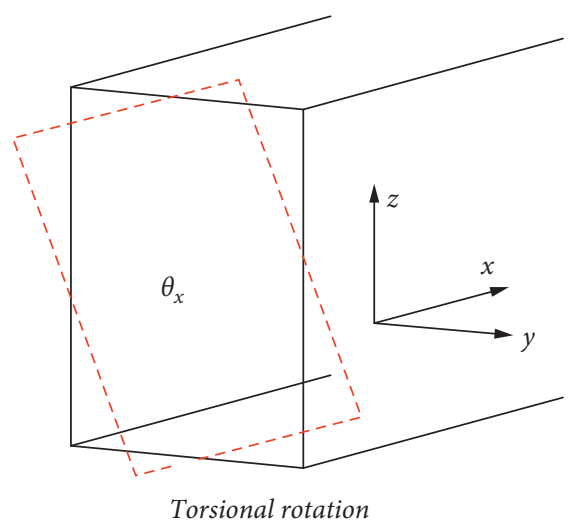

(c)

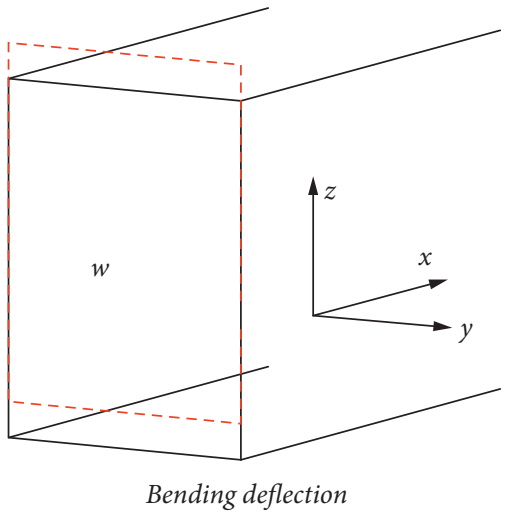

(b)

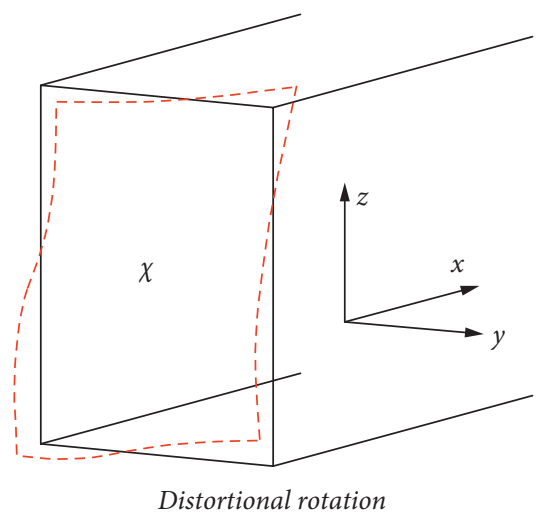

(d)

Figure 2: The transverse displacements.

where $d$ is the additional uniform displacement function modified by the shear lag warping function and $A$ is the cross section area; this method is valid and correct for this paper.

The microelement of generalized distortion displacement in STBB wall is shown in Figure 4. In accordance with any point $N_{0}$ in the middle of plane, the longitudinal, normal, and circumferential displacements of the plane at any point $N_{1}$ can be obtained by ignoring the compression of the wall in the direction of the plane thickness. In the light of middle shear strain $\gamma_{x s}=\left(\partial \psi_{s} \partial_{\chi} / \partial x\right)+\left(-\partial \omega \mathrm{d} \chi^{\prime} / \partial s\right)=0$, the 


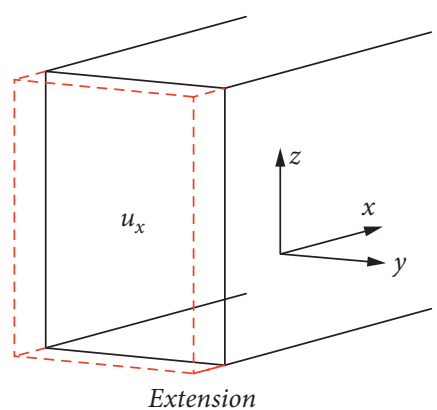

(a)

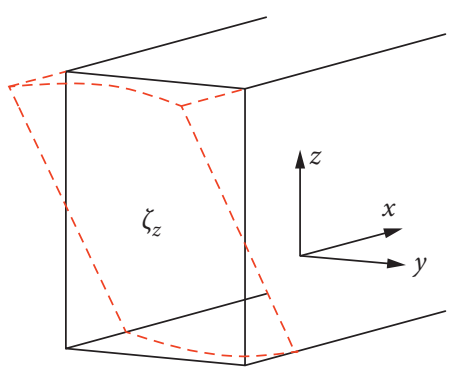

Shear lag deflection

(d)

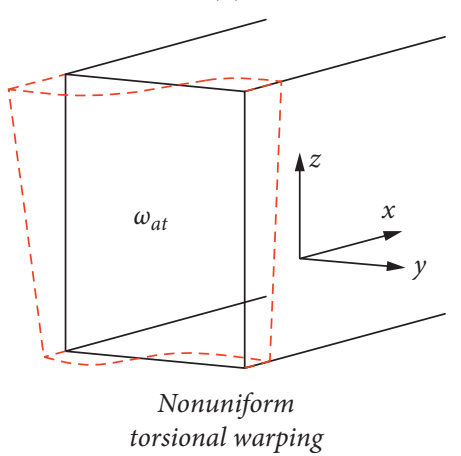

(g)

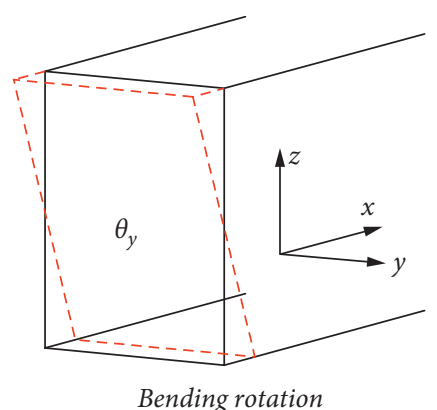

(b)

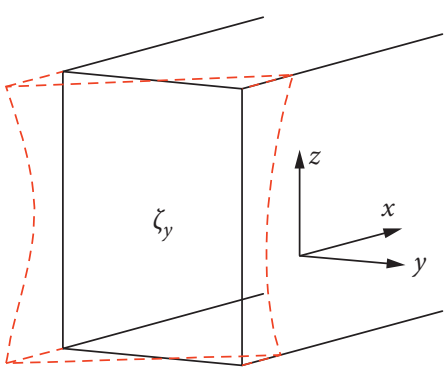

Shear lag deflection

(e)

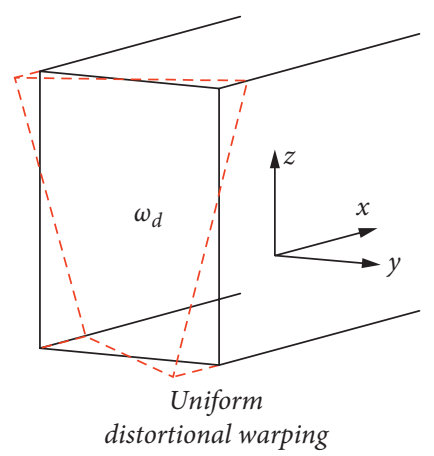

(h)

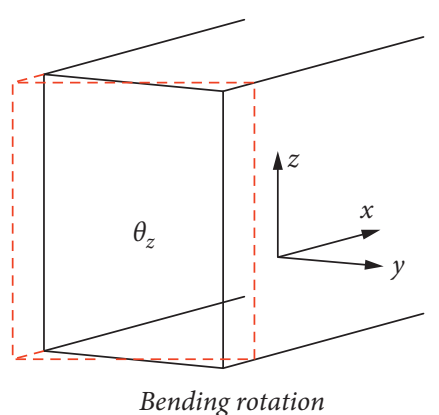

(c)

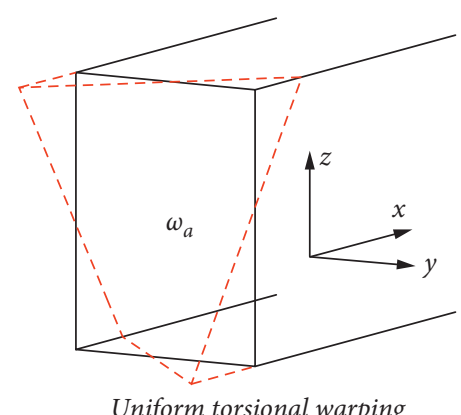

(f)

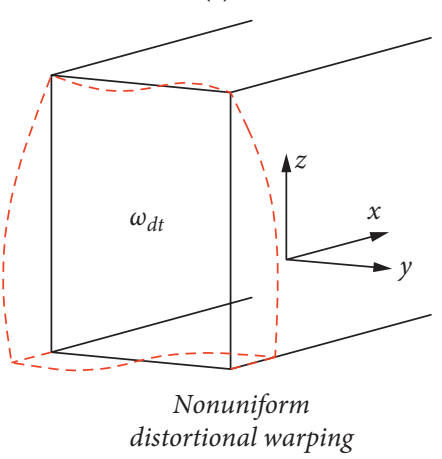

(i)

Figure 3: The longitudinal displacements $u(y, z, x), v(y, z, x)$, and $w(y, z, x)$.

distortion warping can be expressed as $\omega_{d}(s)=\int_{s}^{0} \psi_{s}(s) \mathrm{d} s$. From the self-equilibrium condition of the warping stress, $\int_{A} \omega_{d} \mathrm{~d} A=0$.

In the case that the box girder has no torsion but only distortion deformation, the interposition pure torsion force system $1 / \Omega$ is added at both ends of the microsection $\mathrm{d} x$ of STBB and the circumferential displacement $\psi_{s} \delta \chi$ of the distorted microsection is taken as the virtual displacement. Then, the virtual work done by the unit pure torque force on the virtual displacement is zero: $\int\left[(1 / \Omega) \psi_{s}(s) \delta_{\chi}\right] \mathrm{d} s=0$, that is, $\int \psi_{s}(s) \mathrm{d} s=0$. Through the frame angular point displacement and the continuity of bending moment, the coefficients can be obtained in reference [21]:

$$
\begin{aligned}
\psi_{s}^{i}(s) & =\xi_{1}^{2}\left(\frac{h}{2}+\xi_{1} z_{0}\right)+\xi_{2}^{2}\left(\frac{b}{2}-\xi_{2} y_{0}\right), \\
\psi_{n}^{i}(s) & =\left(-\frac{L_{\text {side }}}{2}+\frac{3}{L_{\text {side }}} s^{2}-\frac{2}{L_{\text {side }}^{2}} s^{3}\right)(-1)^{i+1} \alpha, \\
\alpha & = \begin{cases}\frac{2 b}{b+h}, \quad i=1,3, \\
\frac{2 h}{b+h}, \quad i=2,4,\end{cases}
\end{aligned}
$$

where $L_{\text {side }}$ is the length of each wall of the cross section and $\alpha$ is a coefficient to satisfy the static force equilibrium. The displacement of STBB is given by 


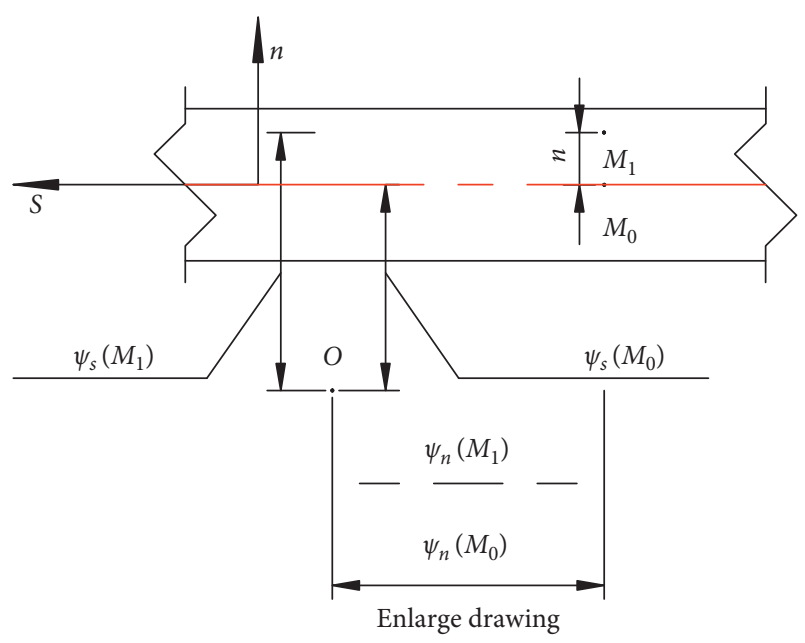

FIGURE 4: The distortion displacement of microelement.

$$
\begin{aligned}
u^{i}(s, x, n)= & u_{0}(x)+Z^{i}(s) w_{0}^{\prime}(x)+Y^{i}(s) v_{0}^{\prime}(x) \\
& -\omega_{\zeta_{z}}^{i}(s) \zeta_{z}(x)-\omega_{a}^{i}(s) \theta^{\prime}(x)-\omega_{d}^{i}(s) \chi^{\prime}(x), \\
v^{i}(s, x, n)= & \xi_{1}^{i} v_{0}(x)-\xi_{1}^{i} w_{0}(x)-h^{i}(s) \theta(x)+\eta^{i}(s, n) \chi(x),
\end{aligned}
$$

$w^{i}(s, x, n)=-\xi_{1}^{i} w_{0}(x)-\xi_{2}^{i} v_{0}(x)-s \theta(x)+\psi_{n}^{i}(s) \chi(x)$,

in which $i=1 \sim 4$ are the wall numbers. The functions $Z^{i}(s)$, $Y^{i}(s), h^{i}(s), \omega_{\alpha}^{i}(s)$, and $\eta(s, n)$ in above equations are expressed as follows:

$$
\begin{aligned}
Z^{i}(s) & =\xi_{1}\left(\frac{h}{2}+\xi_{1} z_{0}\right)+\xi_{2} s, \\
Y^{i}(s) & =\xi_{2}\left(\frac{b}{2}-\xi_{2} y_{0}\right)+\xi_{1} s, \\
h^{i}(s) & =\xi_{1}^{2}\left(\frac{h}{2}+\xi_{1} z_{0}\right)+\xi_{2}^{2}\left(\frac{b}{2}-\xi_{2} y_{0}\right), \\
\omega_{a}^{i}(s) & =\left[\xi_{2}^{2}\left(\frac{b}{2}-\xi_{2} y_{0}\right)-\xi_{1}^{2}\left(\frac{h}{2}+\xi_{1} z_{0}\right)\right] s, \\
\eta^{i}(s, n) & =\psi_{s}^{i}(s)-n \frac{\mathrm{d} \psi_{n}^{i}(s)}{\mathrm{d} s}, \\
\xi_{1} & =\frac{1+(-1)^{i+1}}{2}(i-2), \\
\xi_{2} & =\frac{1+(-1)^{i}}{2}(3-i) .
\end{aligned}
$$

2.4. Expressions of Strains and Stresses. In order to explain the strain tensor of STBB, the normal and shear strains containing the linear and nonlinear strains are expressed as follows:

$$
\begin{aligned}
\varepsilon_{x x}^{i}= & \frac{\partial u^{i}(s, x, n)}{\partial x}+\frac{1}{2}\left[\left(\frac{\partial u^{i}(s, x, n)}{\partial x}\right)^{2}+\left(\frac{\partial v^{i}(s, x, n)}{\partial x}\right)^{2}\right. \\
& \left.+\left(\frac{\partial w^{i}(s, x, n)}{\partial x}\right)^{2}\right], \\
\gamma_{x s}^{i}= & \frac{\partial u^{i}(s, x, n)}{\partial s}+\frac{\partial v^{i}(s, x, n)}{\partial x}+\frac{\partial u^{i}(s, x, n)}{\partial x} \frac{\partial u^{i}(s, x, n)}{\partial s} \\
& +\frac{\partial v^{i}(s, x, n)}{\partial x} \frac{\partial v^{i}(s, x, n)}{\partial s}+\frac{\partial w^{i}(s, x, n)}{\partial x} \frac{\partial w^{i}(s, x, n)}{\partial x} .
\end{aligned}
$$

In the case of normal strain, the expression according to the linear and nonlinear conditions can be denoted by $\varepsilon i \cdot l$ and $\varepsilon i \cdot n l$ :

$$
\varepsilon_{x x}^{i}=\varepsilon_{l}^{i}+\varepsilon_{n l}^{i},
$$

where

$$
\begin{aligned}
\varepsilon_{l}^{i}= & u_{0}^{\prime}+Z^{i}(s) w_{0}^{\prime \prime}(x)+Y^{i}(s) v_{0}^{\prime \prime}(x)-\omega_{a}^{i}(s) \theta^{\prime \prime}(x) \\
& -\omega_{d}^{i}(s) \chi^{\prime \prime}(x)-\omega_{\zeta_{z}}^{i}(s) \zeta_{z}^{\prime}(x),
\end{aligned}
$$

$$
\begin{aligned}
\varepsilon_{n l}^{i}= & \frac{1}{2}\left[u_{o}^{\prime}(x)^{2}+v_{0}^{\prime}(x)^{2}+w_{0}^{\prime}(x)^{2}\right. \\
& +\omega_{\zeta_{z}}^{i}(s)^{2} \zeta_{z}^{\prime}(x)^{2}+\left(\eta^{i}(s, n)^{2}+\psi_{n}^{i}(s)^{2}\right) \chi^{\prime}(x)^{2} \\
& +\left(h^{i}(s)^{2}+s^{2}+s^{2}\right) \theta^{\prime}(x)^{2}+Y^{i}(s)^{2} v_{0}^{\prime \prime}(x)^{2} \\
& \left.+Z^{i}(s)^{2} w_{0}^{\prime \prime}(x)^{2}+\omega_{a}^{i}(s)^{2} \theta^{\prime \prime}(x)^{2}+\omega_{d}^{i}(s)^{2} \chi^{\prime \prime}(x)^{2}\right] \\
& -\left(\xi_{1} h^{i}(s)-\xi_{2} s\right) v_{0}^{\prime}(x) \theta^{\prime}(x)+\left(\xi_{2} h^{i}(s)-\xi_{1} s\right) w_{0}^{\prime}(x) \theta^{\prime}(x) \\
& +\left(\xi_{2} \eta^{i}(s, n)+\xi_{2} \psi_{n}^{i}(s)\right) v_{0}^{\prime}(x) \chi^{\prime}(x)-\omega_{\xi_{z}}^{i}(s) u_{0}^{\prime}(x) \zeta_{z}^{\prime}(x) \\
& -\left(\xi_{2} \eta^{i}(s, n)+\xi_{1} \psi_{n}^{i}(s)\right) w_{0}^{\prime}(x) \chi^{\prime}(x) \\
& -\left(h^{i}(s) \eta^{i}(s, n)+s \psi_{n}^{i}(s)\right) \theta^{\prime}(x) \chi^{\prime}(x) \\
& +Y^{i}(s) u_{0}^{\prime}(x) v_{0}^{\prime \prime}(x)-Y^{i}(s) \omega_{\zeta_{z}}^{\prime}(s) v_{0}^{\prime \prime}(x) \zeta_{z}^{\prime}(x) \\
& -\omega_{a}^{i}(s) u_{0}^{\prime}(x) \theta^{\prime \prime}(x)+\omega_{a}^{i}(s) \omega_{\zeta_{z}}^{i}(s) \theta^{\prime \prime}(x) \zeta_{z}^{\prime}(x) \\
& +Z^{i}(s) u_{0}^{\prime}(x) w_{0}^{\prime \prime}(x)-Z^{i}(s) \omega_{\zeta_{z}}^{\prime}(s) w_{0}^{\prime \prime}(x) \zeta_{z}^{\prime}(x) \\
& -\omega_{d}^{i}(s) u_{0}^{\prime}(x) \chi^{\prime \prime}(x)+\omega_{d}^{i}(s) \omega_{\zeta_{z}}^{i}(s) \chi^{\prime \prime}(x) \zeta_{z}^{\prime}(x) \\
& -Y^{i}(s) \omega_{a}^{i}(s) v_{0}^{\prime \prime}(x) \theta^{\prime \prime}(x)+Z^{i}(s) Y^{i}(s) w_{0}^{\prime \prime}(x) v_{0}^{\prime \prime}(x) \\
& -Y^{i}(s) \omega_{d}^{i}(s) v_{0}^{\prime \prime}(x) \chi^{\prime \prime}(x)-Z^{i}(s) \omega_{a}^{i}(s) w_{0}^{\prime \prime}(x) \theta^{\prime \prime}(x) \\
& -Z^{i}(s) \omega_{d}^{i}(s) w_{0}^{\prime \prime}(x) \chi_{0}^{\prime \prime}(x)+\omega_{d}^{i}(s) \omega_{d}^{i}(s) \theta^{\prime \prime}(x) \chi^{\prime \prime}(x) .
\end{aligned}
$$

The shear strain $\gamma_{x s}^{i}$ can be represented as 


$$
\begin{aligned}
\gamma_{x s}^{i}= & -\omega_{\zeta_{z}^{\prime}}^{\prime}(s) \zeta_{z}(x)-\left(\omega_{a}^{\prime}(s)-h(s)\right) \theta^{\prime}(x)-\left(\omega_{d}^{\prime}(s)-\eta(s, n)\right) \chi^{\prime}(x)-\psi_{n}(s) \theta(x) \chi^{\prime}(x) \\
& -\left(h(s) \eta^{\prime}(s, n)+s \psi_{n}^{\prime}(s)\right) \theta^{\prime}(x) \chi(x)+s \theta(x) \theta^{\prime}(x)+\left(\eta(s, n) \eta^{\prime}(s, n) \eta^{\prime}+\psi_{n}(s) \psi_{n}^{\prime}(s)\right) \chi(x) \chi^{\prime}(x) \\
& +\omega_{a}(s) \omega_{\zeta_{z}}^{\prime}(s) \theta^{\prime \prime}(x) \zeta_{z}(x)+\omega_{d}(s) \omega_{\zeta_{z}}^{\prime}(s) \chi^{\prime \prime}(x) \zeta_{z}(x)+\omega_{a}(s) \omega_{d}^{\prime}(s) \theta^{\prime \prime}(x) \chi^{\prime}(x) \\
& +\omega_{d}(s) \omega_{a}^{\prime}(s) \theta^{\prime}(x) \chi^{\prime}(x)+\omega_{a}(s) \omega_{a}^{\prime}(s) \theta^{\prime}(x) \theta^{\prime \prime}(x)+\omega_{d}(s) \omega_{d}^{\prime}(s) \chi^{\prime}(x) \chi^{\prime \prime}(x) .
\end{aligned}
$$

The stresses are carried out by multiplying the normal and shear strain by $E$ (Young's modulus constant) and $G$ (shear modulus constant), respectively.

$$
\begin{aligned}
\sigma_{x x}^{i} & =E \varepsilon_{x x}^{i} ; \\
\tau_{x s}^{i} & =G \gamma_{x s}^{i} .
\end{aligned}
$$

2.5. Variational Formulation. According to the energy variational principle, the total system potential energy is composed of external potential and internal strain energy
( $W$ and $U$ ). Therefore, the first-order expression of the total potential energy of the system is

$$
\begin{aligned}
& \delta(W-U)=0 \\
& \delta U=\sum_{i=1}^{4} \int_{L} \int_{F_{i}}\left(\sigma_{x x}^{i} \delta \varepsilon_{x x}^{i}+\tau_{x x}^{i} \delta \gamma_{x x}^{i}\right) \mathrm{d} F_{i} \mathrm{~d} x .
\end{aligned}
$$

Substitute longitudinal and shear strain equations (10b)-(10d) into the elastic energy equation (13) to get the internal strain energy expression:

$$
\begin{aligned}
& \delta U=\int_{L} N\left(\delta u_{0}^{\prime}+u_{0}^{\prime} \delta u_{0}^{\prime}+v_{0}^{\prime} \delta v_{0}^{\prime}+w_{0}^{\prime} \delta w_{0}^{\prime}\right) \mathrm{d} x+\int_{L} M_{z}\left(\delta v^{\prime \prime}+w_{0}^{\prime} \delta \theta^{\prime}+\theta^{\prime} \delta w_{0}^{\prime}+u_{0}^{\prime} \delta v_{0}^{\prime \prime}+v_{0}^{\prime \prime} \delta u \prime\right) \mathrm{d} x \\
& +\int_{L} M_{y}\left(\delta w^{\prime \prime}+v_{0}^{\prime} \delta \theta^{\prime}+\theta^{\prime} \delta v_{0}^{\prime}+u_{0}^{\prime} \delta w_{0}^{\prime \prime}+w_{0}^{\prime \prime} \delta u \prime\right) \mathrm{d} x-\int_{L} B_{\omega_{a}}\left(\delta \theta_{0}^{\prime}+u_{0}^{\prime} \delta \theta^{\prime \prime}+\theta^{\prime \prime} \delta u_{0}^{\prime}\right) \mathrm{d} x+\int_{L} M_{R} \theta^{\prime} \delta \theta^{\prime} \mathrm{d} x \\
& -\int_{L} B_{\omega_{d}}\left(\delta \chi^{\prime \prime}+u_{0}^{\prime} \delta \chi^{\prime \prime}+\chi^{\prime \prime} \delta u^{\prime}\right) \mathrm{d} x+\int_{L} M_{\psi} \chi^{\prime} \delta \chi^{\prime} \mathrm{d} x-\int_{L} M_{\psi R}\left(\theta^{\prime} \delta \chi^{\prime}+\chi^{\prime} \delta \theta^{\prime}\right) \mathrm{d} x+\int_{L} M_{\psi y}\left(v_{0}^{\prime} \delta \chi^{\prime}+\chi^{\prime} \delta v_{0}^{\prime}\right) \mathrm{d} x \\
& -\int_{L} M_{\psi z}\left(w_{0}^{\prime} \delta \chi^{\prime}+\chi^{\prime} \delta w_{0}^{\prime}\right) \mathrm{d} x-\int_{L}-\int_{L} M_{z \omega_{\zeta_{z}}}\left(\zeta_{z}^{\prime} \delta v_{0}^{\prime \prime}+v_{0}^{\prime \prime} \delta \zeta_{z}^{\prime}\right) \mathrm{d} x+\int_{L} M_{\omega_{a} \omega_{\zeta_{z}}}\left(\zeta_{z}^{\prime} \delta \theta^{\prime \prime}+\theta^{\prime \prime} \delta \zeta_{z}^{\prime}\right) \mathrm{d} x+\int_{L} M_{\omega_{\zeta_{z}}} \delta \zeta_{z}^{\prime} \mathrm{d} x \\
& +\int_{L} M_{\omega_{d} \omega_{\zeta_{z}}}\left(\zeta_{z}^{\prime} \delta \chi^{\prime \prime}+\chi^{\prime \prime} \delta \zeta_{z}^{\prime}\right) \mathrm{d} x+\int_{L} M_{Y^{2}} w_{0}^{\prime \prime} \delta w_{0}^{\prime \prime} \mathrm{d} x+\int_{L} M_{Z^{2}} v_{0}^{\prime \prime} \delta v_{0}^{\prime \prime} \mathrm{d} x+\int_{L} M_{\omega_{a}^{2}} \theta^{\prime \prime} \delta \theta^{\prime \prime} \mathrm{d} x+\int_{L} M_{\omega_{a}^{2}} \chi^{\prime \prime} \delta \chi^{\prime \prime} \mathrm{d} x \\
& +\int_{L} M_{Z Y}\left(w_{0}^{\prime \prime} \delta v_{0}^{\prime \prime}+v_{0}^{\prime \prime} \delta w_{0}^{\prime \prime}\right) \mathrm{d} x-\int_{L} M_{y \omega_{a}}\left(w_{0}^{\prime \prime} \delta \theta^{\prime \prime}+\theta^{\prime \prime} \delta w_{0}^{\prime \prime}\right) \mathrm{d} x-\int_{L} M_{y \omega_{a}}\left(v_{0}^{\prime \prime} \delta \theta^{\prime \prime}+\theta^{\prime \prime} \delta v_{0}^{\prime \prime}\right) \mathrm{d} x \\
& -\int_{L} M_{y \omega_{d}}\left(w_{0}^{\prime} \delta \chi^{\prime \prime}+\chi^{\prime \prime} \delta w_{0}^{\prime \prime}\right) \mathrm{d} x-\int_{L} M_{z \omega_{d}}\left(v_{0}^{\prime \prime} \delta \chi^{\prime \prime}+\chi^{\prime \prime} \delta v_{0}^{\prime \prime}\right) \mathrm{d} x+\int_{L} M_{\omega_{a} \omega_{d}}\left(\chi^{\prime \prime} \delta \theta^{\prime \prime}+\theta^{\prime \prime} \delta \chi^{\prime \prime}\right) \mathrm{d} x \\
& -\int_{L} M_{\omega_{\zeta z}^{\prime}} \delta \zeta_{z} \mathrm{~d} x-\int_{L} M_{s v} \delta \theta^{\prime} \mathrm{d} x+\int_{L} M_{s v \psi} \delta \chi^{\prime} \mathrm{d} x+\int_{L} M_{1}\left(\theta \delta \theta^{\prime}+\theta^{\prime} \delta \theta\right) \mathrm{d} x-\int_{L} M_{2}\left(\theta \delta \chi^{\prime}+\chi^{\prime} \delta \theta\right) \mathrm{d} x \\
& +\int_{L} M_{3}\left(\chi \delta \chi^{\prime}+\chi^{\prime} \delta \chi\right) \mathrm{d} x+\int_{L} M_{4}\left(\theta^{\prime} \delta \chi+\chi \delta \theta^{\prime}\right) \mathrm{d} x+\int_{L} M_{5}\left(\theta^{\prime} \delta \theta^{\prime \prime}+\theta^{\prime \prime} \delta \theta^{\prime}\right) \mathrm{d} c+\int_{L} M_{6}\left(\chi^{\prime} \delta \chi^{\prime \prime}+\chi^{\prime \prime} \delta \chi^{\prime}\right) \mathrm{d} x \\
& +\int_{L} M_{7}\left(\theta^{\prime \prime} \delta \chi^{\prime}+\chi^{\prime} \delta \theta^{\prime \prime}\right) \mathrm{d} x+\int_{L} M_{8}\left(\theta^{\prime} \delta \chi^{\prime \prime}+\chi^{\prime \prime} \delta \theta^{\prime}\right) \mathrm{d} x+\int_{L} M_{\omega_{a} \omega_{\zeta z}^{\prime}}\left(\theta^{\prime \prime} \delta \zeta_{z}+\zeta_{z} \delta \theta^{\prime \prime}\right) \mathrm{d} x+\int_{L} M_{\omega_{a} \omega_{\zeta z}^{\prime}}\left(\zeta_{z} \delta \chi^{\prime \prime}+\chi^{\prime \prime} \delta \zeta_{z}\right) \mathrm{d} x,
\end{aligned}
$$

where $N$ represents axial force, $B_{\omega}$ represents the bimoment, $M_{z}$ and $M_{y}$ represent the bending moments of the $z$ and $y$ directions, respectively, $M_{s v}$ represents St. Venant torsion moment, $M_{\psi}$ represents distortion moment, and $B_{\omega a}$ and $B_{\omega d}$ represent the bimoment and distortion bimoment; similar to $M_{z}$ and $M_{y}, M_{\psi y}$ and $M_{\psi z}$ are distortion warping moments on behalf of the $z$ and $y$ directions; $M_{s v \psi}$ is in reference to St. Venant principle of distortion moment; $M_{R}$ and $M_{\psi R}$ represent high-order stress synthetic; $M_{\omega_{z z}^{2}}$ is shear lag moment; $M_{\omega_{a}^{2}}$ is high-order bimoment; $M_{\omega_{d}^{2}}^{\omega_{z}^{2}}$ is the high-order distortion bimoment; $M_{z \omega \zeta_{z}}$ is shear lag bending moment; $M_{\omega a \omega \zeta z}$ and $M_{\omega d \omega \zeta z}$ are shear lag coupled with torsion and distortion moments, respectively; $M_{z \omega a}$ and $M_{y \omega a}$ are tangential and transverse bimoments; $M_{z \omega d}$ and $M_{y \omega d}$ are tangential and transverse distortion bimoments; $M_{\omega a \omega d}$ is the high-order coupling bimoment; $Q_{\omega_{\zeta z}^{\prime}}$ is the generalized shear force; $M_{1}$ to $M_{8}$ are high-order torques caused by torsion and distortion; $M_{\omega_{a} \omega_{\xi_{z}^{\prime}}}$ is the shear lag coupling torque; and $M_{\omega_{d} \omega_{\zeta_{z}^{\prime}}^{\prime}}$ is the shear lag distortion coupling torque. 


$$
\begin{aligned}
& N=E \int_{A} \varepsilon_{x x} \mathrm{~d} A, \\
& M_{y}=E \int_{A} z \cdot \varepsilon_{x x} \mathrm{~d} A \text {, } \\
& M_{z}=E \int_{A} y \cdot \varepsilon_{x x} \mathrm{~d} A \text {, } \\
& M_{\Psi_{\mathrm{z}}}=E \int_{A}\left(\xi_{1} \Psi_{n}+\xi_{2} \eta\right) \cdot \varepsilon_{x x} \mathrm{~d} A \text {, } \\
& M_{\psi y}=E \int_{A}\left(\xi_{2} \Psi_{n}+\xi_{1} \eta\right) \cdot \varepsilon_{x x} \mathrm{~d} A \text {, } \\
& M_{R}=E \int_{A}\left(h^{2}+s^{2}\right) \cdot \varepsilon_{x x} \mathrm{~d} A, \\
& M_{\psi}=E \int_{A}\left(\psi_{n}^{2}+\eta^{2}\right) \cdot \varepsilon_{x x} \mathrm{~d} A, \\
& M_{\psi R}=E \int_{A}\left(s \psi_{n}+h \eta\right) \cdot \varepsilon_{x x} \mathrm{~d} A \text {, } \\
& B_{\omega_{a}}=E \int_{A} \omega_{a} \cdot \varepsilon_{x x} \mathrm{~d} A, \\
& B_{\omega_{d}}=E \int_{A} \omega_{d} \cdot \varepsilon_{x x} \mathrm{~d} A, \\
& M_{\omega_{\zeta_{z}}}=E \int_{A} \omega_{\zeta_{z}} \cdot \varepsilon_{x x} \mathrm{~d} A, \\
& M_{z \omega_{\zeta_{z}}}=E \int_{A} z \omega_{\zeta_{z}} \cdot \varepsilon_{x x} \mathrm{~d} A \text {, } \\
& M_{\omega_{a} \omega_{\zeta_{z}}}=E \int_{A} \omega_{a} \omega_{\zeta_{z}} \cdot \varepsilon_{x x} \mathrm{~d} A \text {, } \\
& M_{\omega_{d} \omega_{\zeta_{z}}}=E \int_{A} \omega_{d} \omega_{\zeta_{z}} \cdot \varepsilon_{x x} \mathrm{~d} A \text {, } \\
& M_{z^{2}}=E \int_{A} z^{2} \cdot \varepsilon_{x x} \mathrm{~d} A \text {, } \\
& M_{y^{2}}=E \int_{A} y^{2} \cdot \varepsilon_{x x} \mathrm{~d} A \text {, } \\
& M_{\omega_{a}^{2}}=E \int_{A} \omega_{a}^{2} \cdot \varepsilon_{x x} \mathrm{~d} A, \\
& M_{\omega_{d}^{2}}=E \int_{A} \omega_{d}^{2} \cdot \varepsilon_{x x} \mathrm{~d} A \text {, } \\
& M_{z y}=E \int_{A} z y \cdot \varepsilon_{x x} \mathrm{~d} A \text {, } \\
& M_{z \omega_{a}}=E \int_{A} z \omega_{a} \cdot \varepsilon_{x x} \mathrm{~d} A, \\
& M_{y \omega_{a}}=E \int_{A} y \omega_{a} \cdot \varepsilon_{x x} \mathrm{~d} A \text {, } \\
& M_{z \omega_{d}}=E \int_{A} z \omega_{d} \cdot \varepsilon_{x x} \mathrm{~d} A \text {, } \\
& M_{y \omega_{d}}=E \int_{A} y \omega_{d} \cdot \varepsilon_{x x} \mathrm{~d} A \text {, } \\
& M_{\omega_{a} \omega_{d}}=E \int_{A} \omega_{a} \omega_{d} \cdot \varepsilon_{x x} \mathrm{~d} A \text {, }
\end{aligned}
$$

$$
\begin{aligned}
Q_{\omega_{\zeta_{z}}^{\prime}} & =G \int_{A} \omega_{\zeta_{z}}^{\prime} \cdot \gamma_{x s} \mathrm{~d} A, \\
M_{s v} & =G \int_{A}\left(\omega_{a}^{\prime}-h\right) \cdot \gamma_{x s} \mathrm{~d} A, \\
M_{s v \psi} & =G \int_{A}\left(\eta-\omega_{d}^{\prime}\right) \cdot \gamma_{x s} \mathrm{~d} A, \\
M_{1} & =G \int_{A} s \cdot \gamma_{x s} \mathrm{~d} A, \\
M_{2} & =G \int_{A} \psi_{n} \gamma_{x s} \mathrm{~d} A, \\
M_{3} & =G \int_{A}\left(\eta \eta^{\prime}+\psi_{n} \psi \prime\right) \cdot \gamma_{x s} \mathrm{~d} A, \\
M_{4} & =G \int_{A}\left(h \eta^{\prime}+s \psi \prime\right) \cdot \gamma_{x s} \mathrm{~d} A, \\
M_{5} & =G \int_{A} \omega_{a} \omega_{a}^{\prime} \cdot \gamma_{x s} \mathrm{~d} A, \\
M_{6} & =G \int_{A} \omega_{d} \omega_{d}^{\prime} \cdot \gamma_{x s} \mathrm{~d} A, \\
M_{7} & =G \int_{A} \omega_{a} \omega_{d}^{\prime} \cdot \gamma_{x s} \mathrm{~d} A, \\
M_{8} & =G \int_{A} \omega_{a}^{\prime} \omega_{d} \cdot \gamma_{x s} \mathrm{~d} A, \\
M_{\omega_{a} \omega_{\zeta_{z}}^{\prime}} & =G \int_{A} \omega_{a} \omega_{\zeta_{z}}^{\prime} \cdot \gamma_{x s} \mathrm{~d} A, \\
M_{\omega_{d} \omega_{\zeta_{z}}^{\prime}} & =G \int_{A} \omega_{d} \omega_{\zeta_{z}}^{\prime} \cdot \gamma_{x s} \mathrm{~d} A,
\end{aligned}
$$

and detailed expressions of these forces and torques can be found in Appendix A.

The general formulas about of STBB nonlinear and the buckling analysis are obtained when it is subjected to external forces of combined axial load $F_{x}$ and eccentric load $F_{z}$. In the initial transverse buckling, the external energy is given:

$$
\delta W=\int_{L}\left(F_{x} \delta u_{0}(x)+F_{z} \delta w_{r}(s, x)\right) \mathrm{d} x,
$$

in which $W_{r}(s, r)$ stands for the vertical displacement. In consideration of the classical quadratic kinematics hypothesis, the change of external energy can be expressed as

$$
\begin{aligned}
\delta W= & \int_{L} F_{x} \delta u_{0}(x) \mathrm{d} x+\int_{L} F_{z} \delta\left(w_{0}(x)+\zeta_{z}(x)\right) \mathrm{d} x \\
& -\left(e_{z}+e_{y}\right) \int_{L} F_{z}(\theta(x)+\chi(x)) \delta(\theta(x)+\chi(x)) \mathrm{d} x,
\end{aligned}
$$

where $e_{y}$ and $e_{z}$ are eccentricities of the concentrated load $F_{z}$ relative to the shear center.

According to equations (14) and (17), the virtual displacements and their derivatives can be used to express the change of the total potential energy. Due to the generalized variational principle, balance equations with reference to a first-order variation $\partial u(x), \partial v(x), \partial w(x), \partial \theta(x), \partial \chi(x)$, and $\partial \zeta(x)$ can be written as 


$$
\begin{aligned}
& N^{\prime}+\left(N u^{\prime}\right)^{\prime}+M_{y} w^{\prime \prime}+M_{z} v^{\prime \prime}-B_{\omega_{a}} \theta^{\prime \prime}-B_{\omega_{d}} \chi^{\prime \prime}=F_{x} \\
& N v^{\prime}+M_{z}^{\prime}+M_{y} \theta^{\prime}+M_{\psi y} \chi^{\prime}+\left(M_{z} u^{\prime}\right)^{\prime}-\left(M_{z \omega_{\zeta}} \zeta_{z}^{\prime}\right)^{\prime}+\left(M_{z^{2}} v^{\prime \prime}\right)^{\prime}+\left(M_{Z Y} w^{\prime \prime}\right)^{\prime}-\left(M_{z \omega_{a}} \theta^{\prime \prime}\right)^{\prime}-\left(M_{z \omega_{d}} \chi^{\prime \prime}\right)^{\prime}=0 \\
& \left(N w^{\prime}\right)^{\prime}+\left(M_{z} \theta^{\prime}\right)^{\prime}+M_{y}^{\prime \prime}-\left(M_{\psi_{z}} \chi^{\prime}\right)^{\prime}+\left(M_{y} u^{\prime}\right)^{\prime \prime}+\left(M_{Y^{2}} w^{\prime \prime}\right)+\left(M_{Z Y} v^{\prime \prime}\right)^{\prime \prime}-\left(M_{y \omega_{a}} \theta^{\prime \prime}\right)^{\prime \prime}-\left(M_{y \omega_{d}} \chi^{\prime \prime}\right)^{\prime \prime}=F_{z}, \\
& -B_{\omega_{a}}^{\prime \prime}+\left(M_{y} v^{\prime}\right)^{\prime}+\left(M_{R} \theta^{\prime}\right)-\left(M_{\psi_{R}} \chi^{\prime}\right)^{\prime}-\left(B_{\omega_{a}} u^{\prime}\right)^{\prime \prime}+\left(M_{\omega_{a} \omega_{\zeta}} \zeta_{z}^{\prime}\right)^{\prime \prime}+\left(M_{\omega_{a}^{2}} \theta^{\prime \prime}\right)^{\prime \prime}-\left(M_{y \omega_{a}} w^{\prime \prime}\right)^{\prime \prime}-\left(M_{z \omega_{a}} v^{\prime \prime}\right)^{\prime \prime} \\
& +\left(M_{\omega_{a} \omega_{d}} \chi^{\prime \prime}\right)^{\prime \prime}-M_{s v}^{\prime}+\left(M_{s} \theta\right)^{\prime}+M_{1} \theta^{\prime}-M_{2} \chi^{\prime}-\left(M_{4} \chi\right)^{\prime}+\left(M_{5} \theta^{\prime}\right)^{\prime \prime}+\left(M_{5} \theta^{\prime \prime}\right)^{\prime}+\left(M_{7} \chi^{\prime}\right)^{\prime \prime}+\left(M_{8} \chi^{\prime \prime}\right)^{\prime} \\
& +\left(M_{\omega_{a} \omega_{\zeta_{z}}^{\prime}}\right)^{\prime \prime}=-F_{z}\left(e_{z}+e_{y}\right)(\theta+\chi) \\
& -B_{\omega_{d}}^{\prime \prime}+\left(M_{\psi} \chi^{\prime}\right)^{\prime \prime}+\left(M_{\psi_{y}} v^{\prime}\right)-\left(M_{\psi_{z}} w^{\prime}\right)^{\prime}-\left(M_{\psi_{R}} \theta^{\prime}\right)^{\prime}-\left(B_{\omega_{d}} u^{\prime}\right)^{\prime \prime}+\left(M_{\omega_{d} \omega \zeta_{z}} \zeta_{z}^{\prime}\right)^{\prime \prime}+\left(M_{\omega_{d}^{2}} \chi^{\prime \prime}\right)^{\prime \prime}-\left(M_{y \omega_{d}} w^{\prime \prime}\right)^{\prime \prime} \\
& -\left(M_{z \omega_{d}} v^{\prime \prime}\right)^{\prime \prime}+\left(M_{\omega_{a} \omega_{d}} \theta^{\prime \prime}\right)^{\prime \prime}+M_{s v \psi}^{\prime}+\left(M_{3} \chi\right)^{\prime}+M_{3} \chi^{\prime}-\left(M_{2} \theta\right)^{\prime}-M_{4} \theta^{\prime}+\left(M_{6} \chi^{\prime}\right)^{\prime \prime}+\left(M_{6} \chi^{\prime \prime}\right)^{\prime}+\left(M_{7} \theta^{\prime \prime}\right)^{\prime} \\
& +\left(M_{8} \theta^{\prime}\right)^{\prime \prime}+\left(M_{\omega_{d} \omega_{\zeta_{z}}^{\prime}} \zeta_{z}\right)^{\prime \prime}=-F_{z}\left(e_{z}+e_{y}\right)(\theta+\chi) \\
& M_{\omega_{\zeta_{z}}}^{\prime}+\left(M_{\omega_{\zeta_{z}}}^{\prime} u^{\prime}\right)^{\prime}+\left(M_{y \omega_{\zeta_{z}}} w^{\prime \prime}\right)^{\prime}+\left(M_{z \omega_{\zeta_{z}}} v^{\prime \prime}\right)^{\prime}-\left(M_{\omega_{d} \omega_{\zeta}} \chi^{\prime \prime}\right)^{\prime}+Q_{\omega_{\zeta_{z}}}-M_{\omega_{d} \omega_{\zeta_{z}^{\prime}}} \theta^{\prime \prime}-M_{\omega_{d} \omega_{\zeta_{z}}} \chi^{\prime \prime}=F_{z} \\
& \frac{u(x)}{u_{0}}=\cos \frac{\pi x}{L} \\
& \frac{v(x)}{v_{0}}=\frac{w(x)}{w_{0}}=\frac{\theta(x)}{\theta_{0}}=\frac{\chi(x)}{\chi_{0}}=\frac{\zeta_{z}(x)}{\zeta_{z 0}}=\sin \frac{\pi x}{L},
\end{aligned}
$$
And the buckling load is first determined according to the singularity condition of the tangential stiffness matrix determinant. The discrete kinematic equation can be used to analyze the performance of simply supported structures. The displacement modes adopt the approximate functions that are consistent with the boundary conditions of STBB:

where $u_{0}, v_{0}, w_{0}, \theta_{0}, \chi_{0}$, and $\zeta_{z 0}$ are related to displacement amplitudes. In addition, these functions can also represent exact solutions of nonlinear equilibrium equations. Through Galerkin's approximation method and integration, the algebraic coupling equilibrium equations of displacement modulus can be obtained:

$$
\begin{aligned}
& P_{1} u_{0}+P_{2} w_{0} \zeta_{z 0}+P_{3} u_{0} w_{0}^{2}=\left(\frac{4 L^{2}}{\pi^{2}}\right) F_{x} \\
& P_{4} v_{0}+P_{5} \chi_{0}+P_{6} w_{0} \theta_{0}+P_{7} w_{0} \chi_{0}+P_{8} v_{0} w_{0}^{2}=0 \\
& P_{9} w_{0}+P_{10} \chi_{0}+P_{11} w_{0} \chi_{0}+P_{12} w_{0}^{3}-\left(\frac{16 L}{\pi^{3}}\right) M_{0}=0 \\
& P_{13} \theta_{0}+P_{14} \chi_{0}+P_{15} v_{0} w_{0}+P_{16} w_{0} \chi_{0}+P_{17} \omega_{0}^{2}+P_{18} \omega_{0}^{2} \theta_{0}+P_{19} w_{0}^{2} \chi_{0}+\frac{4\left(e_{y}+e_{z}\right) L}{\pi^{2}} M_{0}=0 \\
& P_{20} w+P_{21} v+P_{22} \theta_{0}+P_{23} \chi_{0}+P_{24} v_{0} w_{0}+P_{25} w_{0} \theta_{0}+P_{26} w_{0} \chi_{0}+P_{27} w_{0}^{2}+P_{28} w_{0}^{2} \theta_{0}+P_{29} w_{0}^{2} \chi_{0}+\frac{4\left(e_{y}+e_{z}\right) L}{\pi^{2}} M_{0}=0 \\
& P_{30} \zeta_{z 0}-\left(\frac{16 L}{\pi^{3}}\right) M_{0}=0
\end{aligned}
$$


in which $M_{0}=F_{z} L / 4$ represents the maximal bending moment when the eccentric load is applied at the middle length of the beam. The detailed expressions of $P_{i}$ algebraic system is shown in Appendix B.

2.7. Tangential Stiffness Matrix for Lateral Buckling. The beam deflects under the action of axial and bending loads. If the beam is loaded to a certain extent, its cross section rotates around the axis of the beam, at which time lateral buckling may occur. This is transverse buckling, and the buckling load is the critical load. Corresponding to the prebuckling state, the deflection is called the "fundamental state."

On account of the Jacobian Matlab function, the tangent stiffness matrix $\left[K_{t}\right]$ is automatically defined by the equilibrium equations (20a)-(20f). In the case of nonlinear highcoupling high-order model and considering the prebuckling state, it can be reasonably assumed that each displacement $\left(u_{0}, v_{0}, w_{0}, \theta_{0}, \chi_{0}, \zeta_{z 0}\right)$ is approximately equal to $\left(0,0, w_{0}, 0,0\right.$, $0)$ by analyzing the geometric matrix. Here, the tangent stiffness matrix of the orthogonal displacement modes is

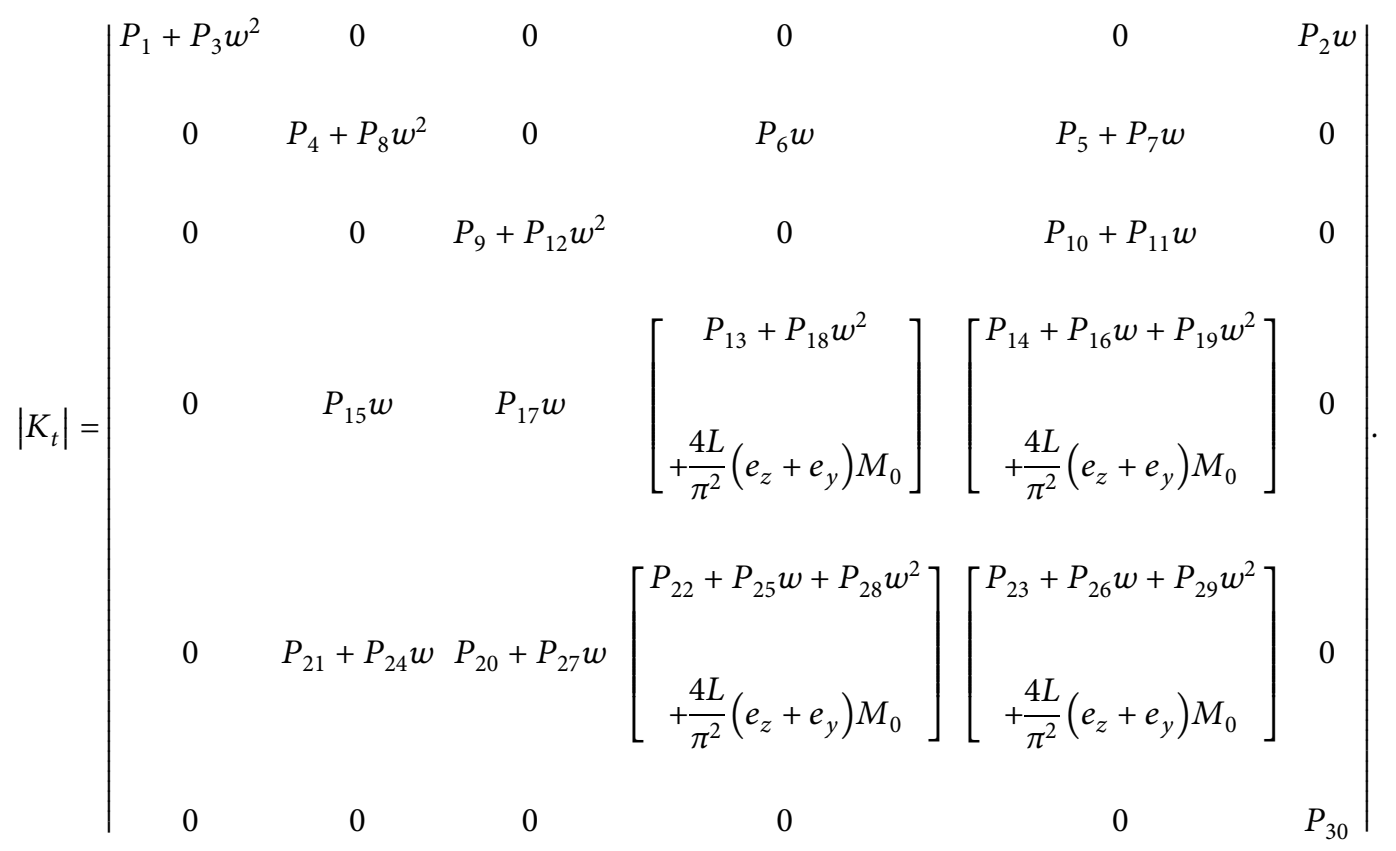

Finally, after eliminating the determinant of the tangent stiffness matrix, we get polynomial equations containing $F_{x}$ and $F_{z}$. In this paper, the roots of the equation yield to transverse buckling loads in different buckling modes (extension, bending, torsion, distortion, and shear lag modes).

\section{Application and Numerical Examples}

The goal of the section is to verify the current theoretical model of STBB buckling behavior so as to demonstrate the accuracy and practical application of the formulas. At the same time, the results are compared with those calculated by FEA (Ansys software), Meftah, and Tan.

The analysis procedure for the nonlinear buckling analysis of STBB is the same as the general nonlinear analysis process. In the Ansys software, the STBB is idealized by the 4-node 181 isoparametric quadrangle shell elements (Figure 5). First, a "perfect" model is established, and the sparse matrix solver is used to conduct eigenvalue buckling analysis, which can predict the critical buckling force and provide a basis for the nonlinear buckling analysis. The grid can generate 3888 nodes and 3840 elements. Meanwhile, the grid is verified to discretize these patterns accurately. In the following analysis, perturbation is introduced in the geometry by adding geometric imperfections using the UPGEOM command. The geometric nonlinear analysis of structures with geometric perturbation is conducted using the arclength method, where the postbuckling behavior can be obtained. The size of the perturbations is usually a small percentage of a relative structure, and the relevant scaling factor used in this case is 0.001 . Finally, the bifurcation point of the buckling load displacement curve is found out, which is the critical buckling load.

In Ref. [33], Meftah studied the lateral buckling of thinwalled box girder under the combined action of bending and axial loads. And in the stress calculation, the nonlinear higher-order terms were omitted. Similarly, in Ref. [25], Tan further analyzed nonlinear lateral buckling of STBB's webs with the unequal thickness, but the main difference was that the geometrically nonlinear high-order coupled terms $\left(w \theta^{2}, w \chi^{2}, w^{2} \theta \ldots\right)$ had been taken into account. Both of them did not consider the shear deformation and the shear lag effect.

3.1. Example 1: Variation of Length and Cross Section. Figure 6 shows a simply supported beam under combined eccentric and axial load. The critical load $F_{z}$ imposed in the 


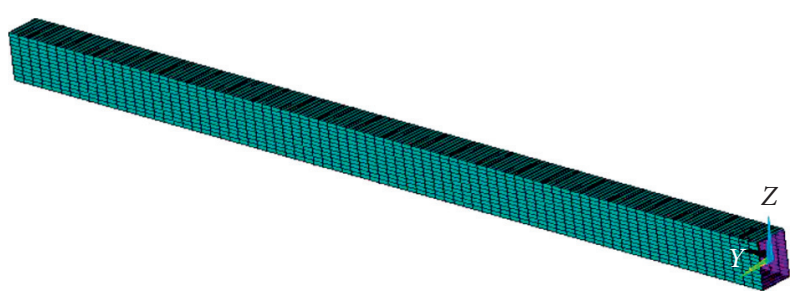

FIGURE 5: Grid division of quadrilateral shell of STBB with unequal thickness webs.

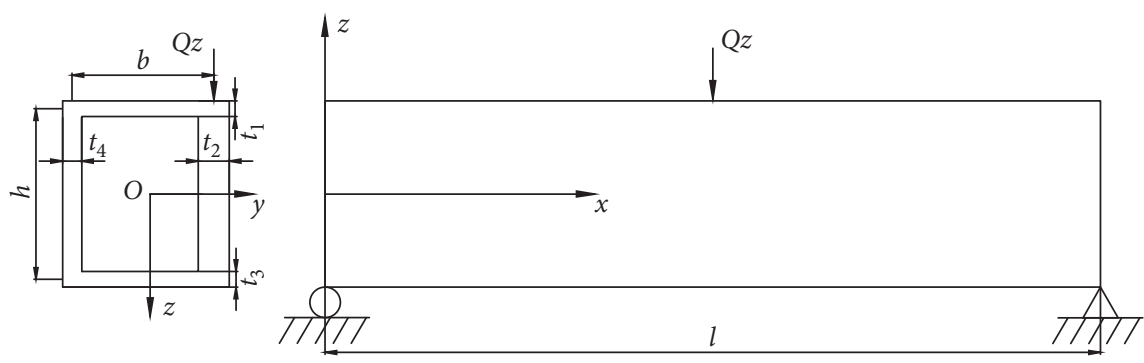

FIGURE 6: The geometry of STBB under combined axial and bending loads.

TABLE 1: Cross section dimension of the models.

\begin{tabular}{lccccc}
\hline Category & I & II & III & IV & V \\
\hline$b(\mathrm{~mm})$ & 200 & 200 & 150 & 150 & 100 \\
$h(\mathrm{~mm})$ & 100 & 150 & 150 & 200 & 200 \\
\hline
\end{tabular}

middle of beam length takes action on the intersection of the upper flange and the center line of the main web, and the axial load $F_{x}$ is applied on one end of the upper flange. The geometrical and material properties are $F_{x}=2.5 \mathrm{kN}$, $E=210 \mathrm{GPa}, t_{1}=t_{3}=4 \mathrm{~mm}, t_{2}=8 \mathrm{~mm}$, and $t_{4}=5 \mathrm{~mm}$. Poisson's ratio is 0.3 . The length ranges from 1.0 to $8.0 \mathrm{~m}$. In addition, in order to illustrate the influence of the lengthwidth ratio of cross section on nonlinear lateral buckling of beams, five different groups of cross sections are selected in this paper. Table 1 shows the detailed cross section dimension of the models.

Figures 7-11 illustrate the comparison between the present and other three theories under different lengths and cross section ratios. The parameters of the beam can indicate the sufficient effectiveness and accuracy of the method in this paper. Tables 2-4 report percentage differences of the displacements $\left(\Delta u_{i}, \Delta w_{i}, \Delta v_{i}\right)$ obtained by theoretical methods and those obtained by FEA. Where, $i=1 \sim 3$ represents the present, Meftah, and Tan's methods, respectively.

As can be seen from Figure 7, the numerical values of the longitudinal displacement are almost between the values of Meftah and FEA. It is easy to see from the picture that Meftah's axial displacements are independent of the change of cross section. At the same length, the longitudinal displacements of the other three methods increase gradually with the decrease of cross section ratio $(b / h)$. It should be noted that the relative errors of present method are the smallest while those of Meftah's method are the largest in Tables 2-4. It is shown that ignoring the shear lag effect will lead to an overestimation of percentage of the longitudinal displacements at any length. With reference to Tan's beams, the values $\Delta u_{1}$ and $\Delta u_{3}$ are the closest, when the aspect ratio of cross section is 1 . This is due to the geometrical nonlinear effect caused by bending, torsion, ,distortion and shear lag coupling, which leads to a decrease in the axial stiffness of STBB. It is confirmed that the axial displacements of the present theory are sufficiently close to those predicted by FEA. Especially in IV and V, the values of present method are closest to those of FEA when the length is under 7 and $8 \mathrm{~m}$ (the smallest error $\Delta u_{1}=6.6 \%$ ). This shows that adding additional shear lag displacement is effective for the prediction of longitudinal displacement.

It can be seen from Figure 8 that the vertical displacement deformation trend is completely corresponding to the rigid body performance, which is true for all models. The results of the present theory are confirmed to be closer to those of FEA, which supports the validity of this theory. Comparing the present theory with the other two, the results are satisfactory and can be considered as a supplement to the shear lag. In the same case, the values of FEA are always greater than those of other methods, with the lowest values of Meftah. Because the three theoretical approaches do not take the vertical compressibility of webs into account under vertical load and more nonlinear factors are considered, the vertical displacement decreases. According to the relative errors of vertical displacements from Table 3, when the beam length is less than $2 \mathrm{~m}$, the shear lag effect has little influence on the vertical displacement of STBB. When the beam tends to be slender, the percentage of vertical displacements is smaller $\left(\Delta w_{1}\right.$ are almost all less than $\left.10 \%\right)$. The present method can well capture the effect of shear deformation on the beam, so it has high prediction accuracy.

In Figure 9, we note that the transversal displacement distribution curve obtained by the present theory is in good agreement with the results obtained by the FEA, which indicates that the proposed beam theory can accurately 


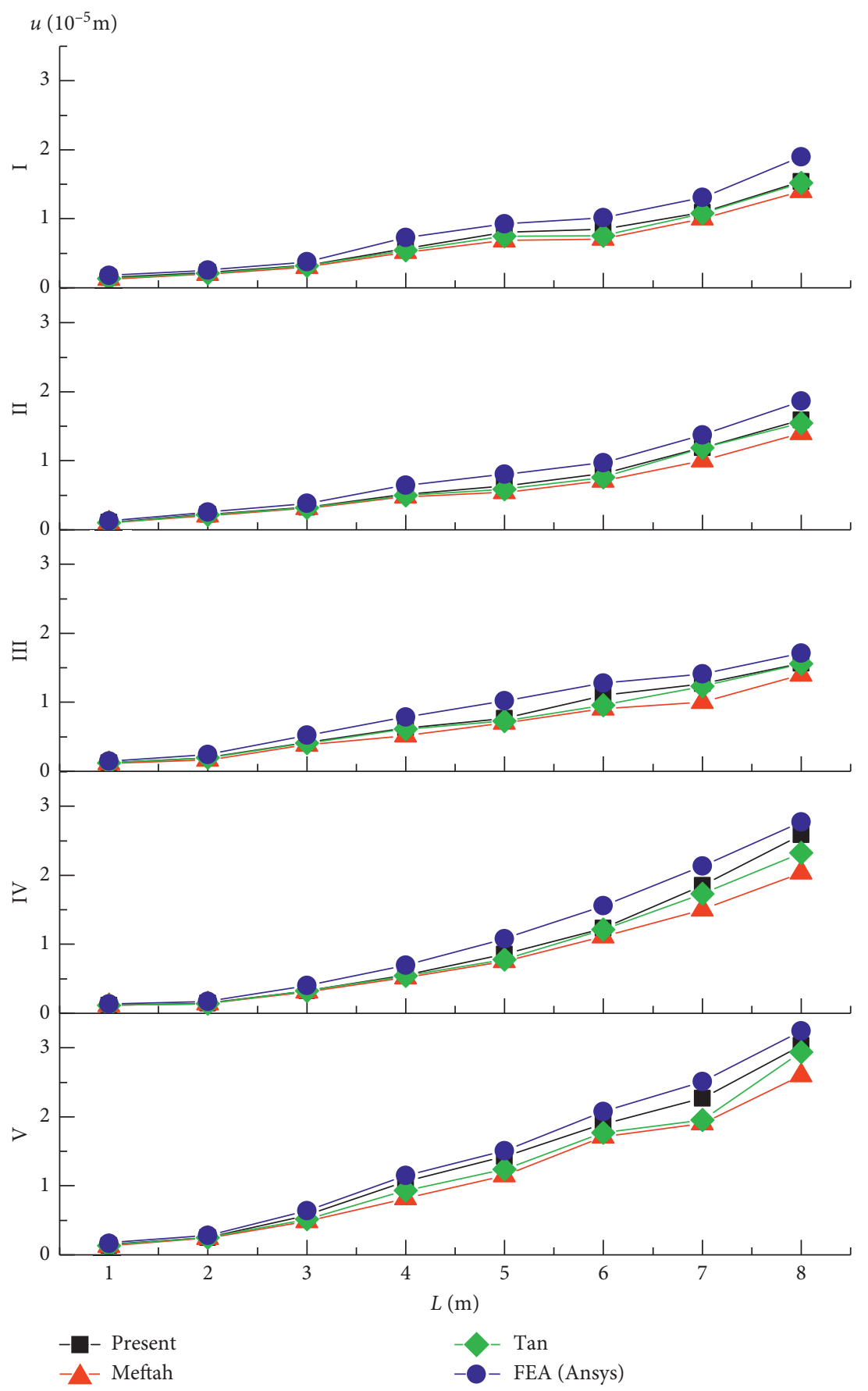

FIgURE 7: Axial displacement comparison.

predict the structural behavior of STBB under different cross sections. It shows that the postbuckling equilibrium paths are stable. In I $(b / h=2 / 1)$, the change of transversal displacement is tiny and the curve is relatively stable when the length is more than $3 \mathrm{~m}$. Again, the same thing happens in III when the length is greater than $5 \mathrm{~m}$. In the other three cases, the curve can be considered as increasing almost linearly. It can be seen that the five displacement modes without considering the shear lag effect are consistent with the prediction of the transverse displacement by present theory. In Table 4, the percentages of transverse displacement of present method are closer than those of the other methods, indicating that the shear lag has a greater impact on the large ratio of the cross section. The results show that although six modes need to deal with more complex deformation relations to obtain the characteristics of transverse, the beam under different lengths and cross sections can be calculated arbitrarily and accurately.

Furthermore, as expected, Tables $2-4$ point out that the relative displacement errors of Meftah are the largest. The shear lag displacement has a significant influence on the displacements of the long beam $(L>3 \mathrm{~m})$, but a slight 


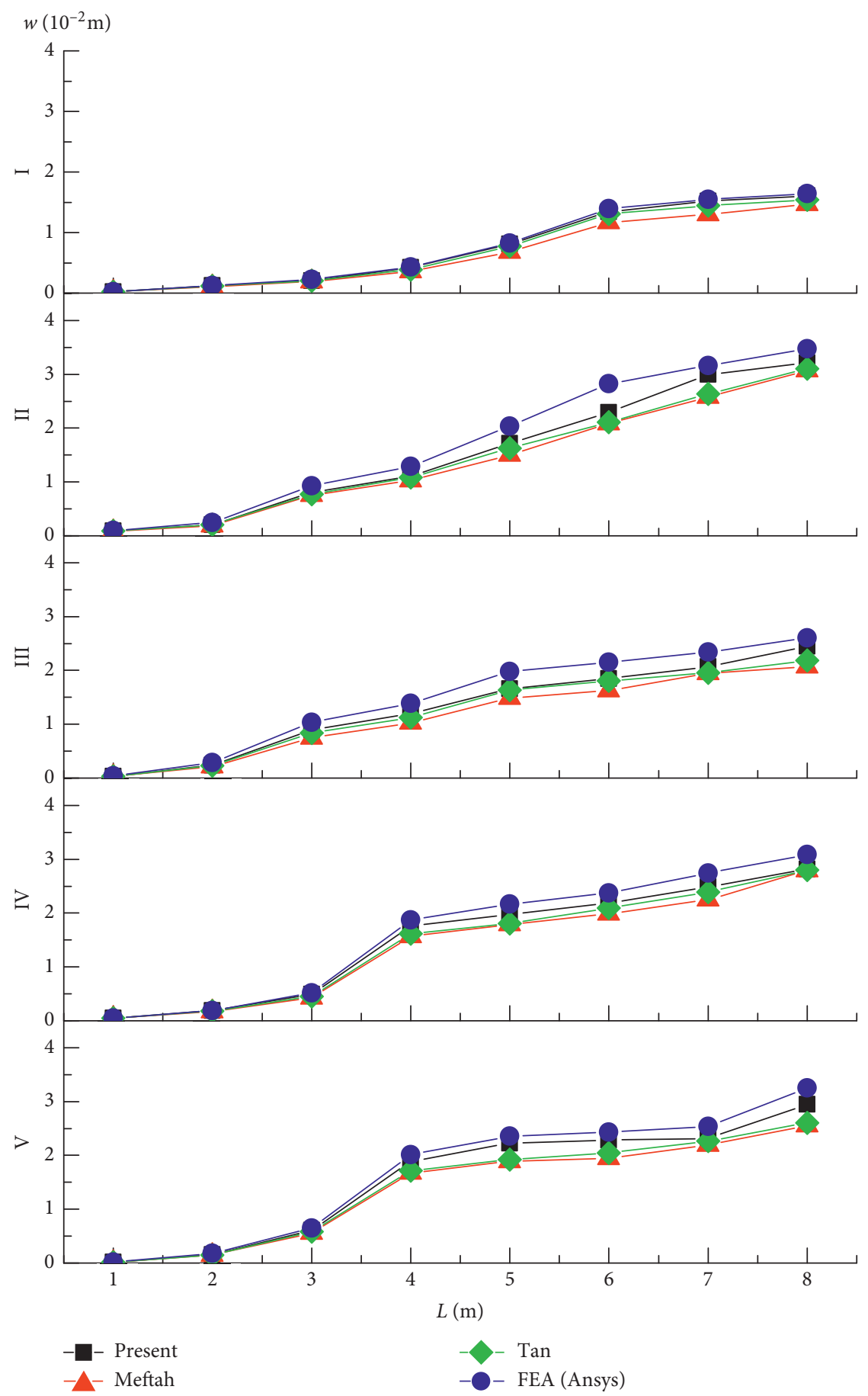

FIGURE 8: Vertical displacement comparison.

influence on the short beam $(L \leq 3 \mathrm{~m})$. The results highlight that the influence of shearing deformation on displacement is great.

In Figure 10, the present results confirm that the proposed methodology agrees well with that of the shell analysis. When the length-width ratio is greater than 1, Meftah's torsional rotations are always bigger than other values, but when the length-width ratio is less than 1, those of FEA become the maximum. Because of ignoring the effect of shear deformation according to Meftah's theory, torsional stiffness is underestimated. When the length is more than $3 \mathrm{~m}$, the torsional rotations start to become more sensitive. Interestingly, when the length between $4 \mathrm{~m}$ and $6 \mathrm{~m}$, the torsional rotations grow slowly and the curve flattens out. The calculated values of Tan are always slightly larger than those of Meftah. And the variation of the two is more moderate with the length. It is easy to see from Figure 10 that the present torsional rotations are smaller than those of other methods, especially when the length-width ratio is less than 1 . The torsion rotations of FEA are slightly different 


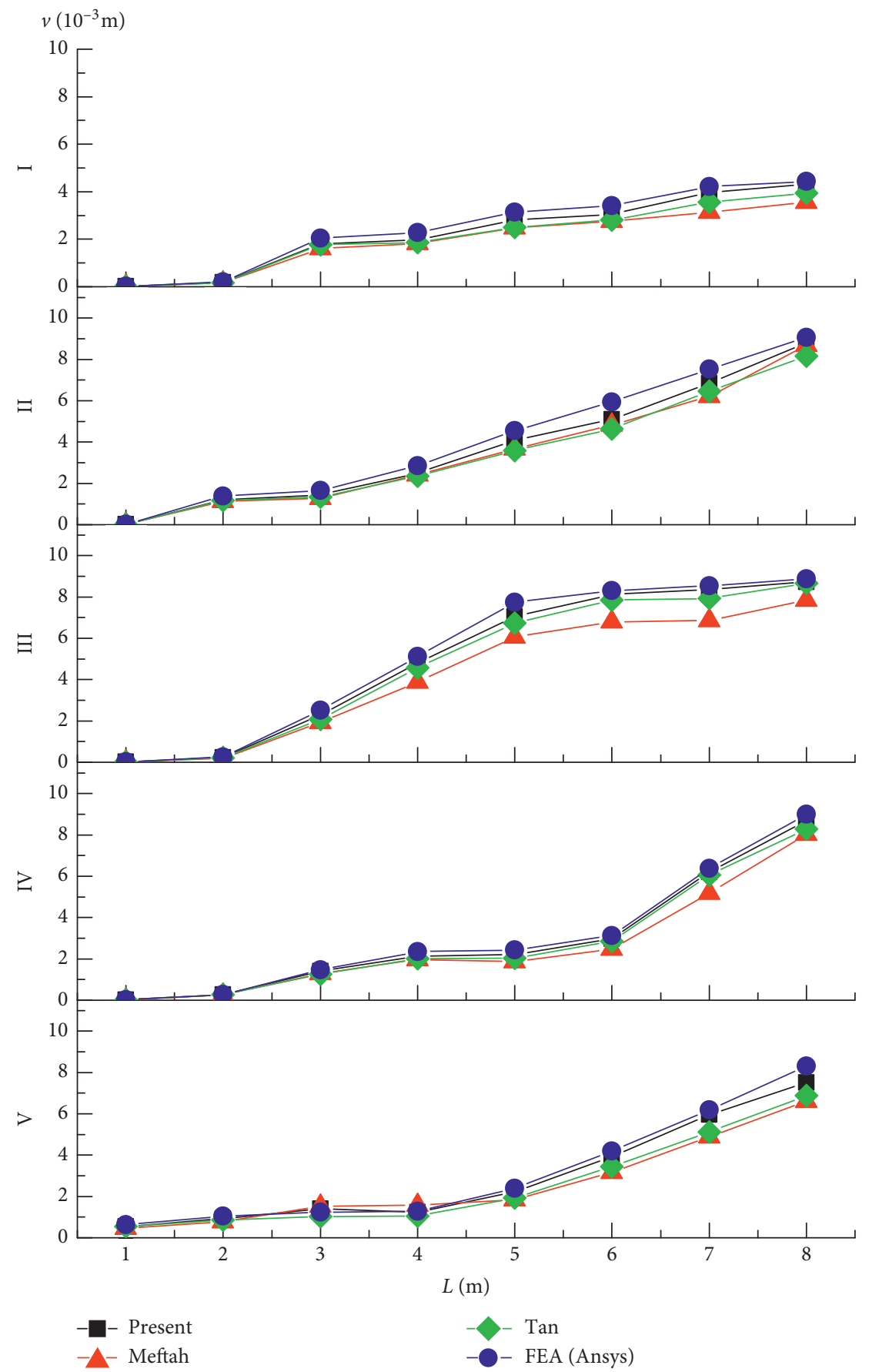

Figure 9: Transversal displacement comparison.

from those of the present model, even in the distribution pattern. The reason is that the shear deformation effect is considered in present model (6 modes), and the shear stress terms not only include the generalized shear lag deformation, torsion, and distortion shear stress terms but also include the coupling torque of torsion and distortion.

The variation trends of distortion rotation of the three methods are in good agreement with each other in Figure 11. The present distortion curve is similar to that of Tan. The results of this theory (6 models) are satisfactory, especially when $b / h \leq 1$, and the curve value is always slightly smaller than that of Tan. In the case of the same length, the distortional rotations of IV case $(b / h=3 / 4)$ are smaller than those of other cross section states. In fact, shear effect reduces the distortion value of the short beam relative to the nonshearable theory. The distortion analysis with shear lag effect shows a conservative behavior compared with a more comprehensive distortion analysis of nonlinear terms.

Figure 12 shows the shear lag warping displacement. It can be seen that the curve trend under the different cross sections is in good agreement within this length range. In general, the shear lag warping displacements gradually increase with the increase of 


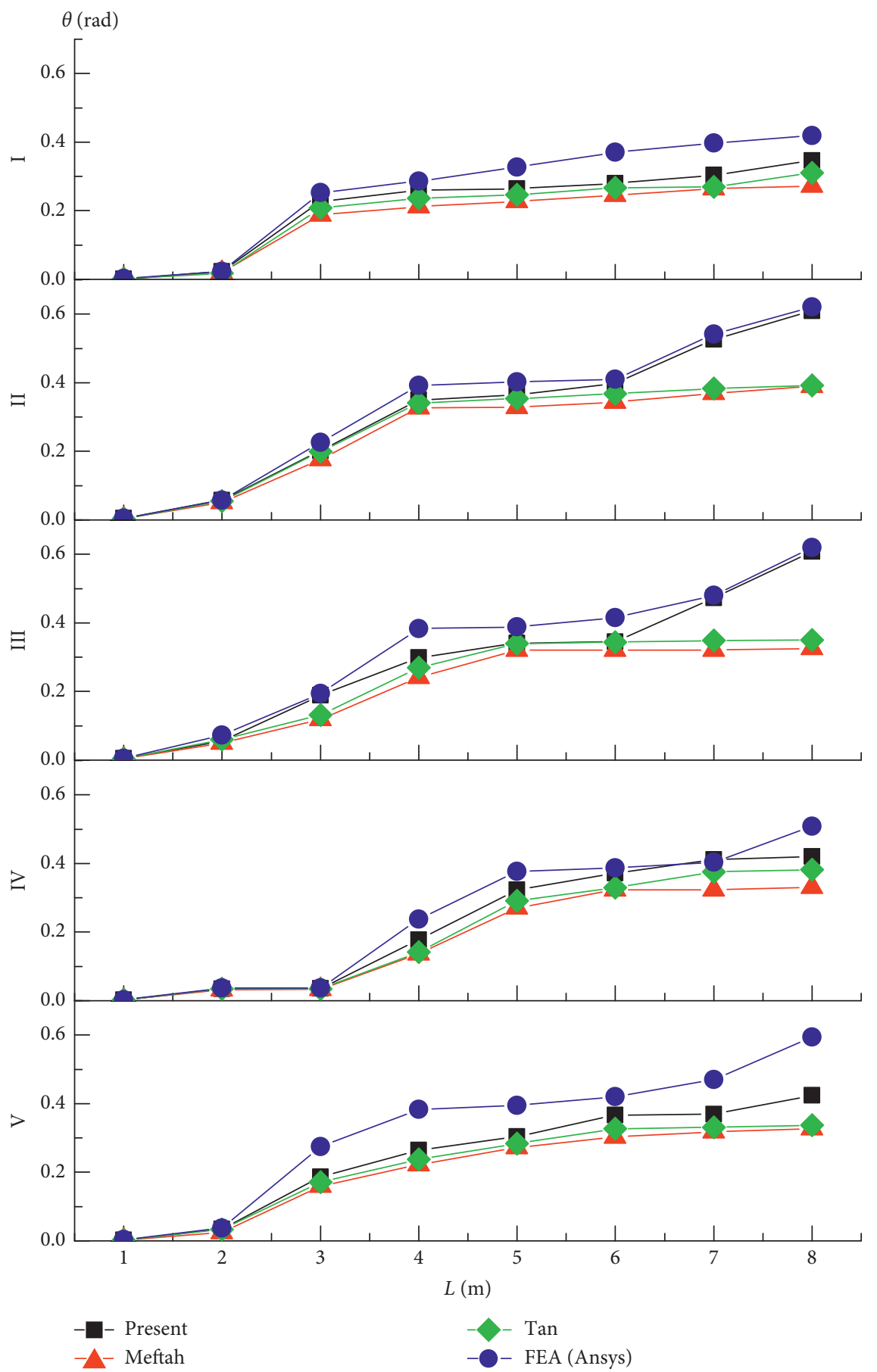

FIgURE 10: Torsional rotation comparison.

length. For $2 \leq L \leq 6$, the relative rate of change is very small $\left(\zeta_{z \min }=3.9287 E-4 m, \zeta_{z \max }=7.8926 E-4 m\right)$. In the same case, the larger the length-width ratio is, the larger the shear lag warping displacement is. We can observe that the curve almost tends to a flat line in $I(b / h=1 / 2)$. In the case of long beams, the shear lag effect has a significant effect on the buckling behavior.

From Figures 7-12, it can be observed that the influence of shear deformation on buckling behavior is more significant when the length increases from $4 \mathrm{~m}$. Due to the influence of defects, the mechanical properties of STBB by the present method are obviously lower than those of FEA. When the cross section length-width ratio is $3 / 4$, the geometric nonlinearity of STBB is optimal. Therefore, the theory presented in this paper is valid for the thin-wall aspect ratio assumed in the next example.

3.2. Example 2: Variation of Height and Thickness. In this numerical example, the effects of beam length, web thickness, and load height on lateral resistance of STBB are studied. Figure 13 shows three different load positions. 


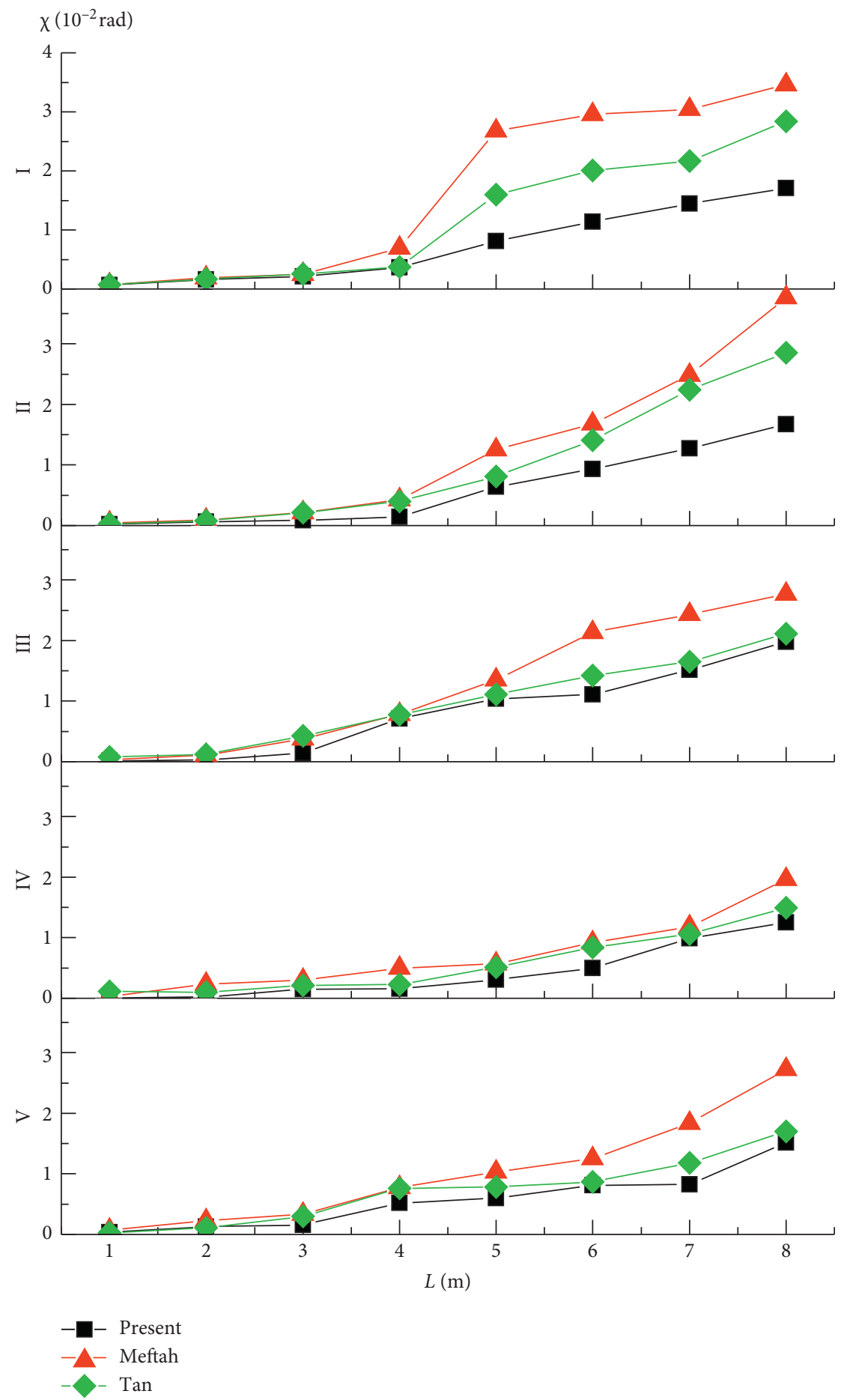

FIGURE 11: Distortional rotation comparison.

Considering the appropriate combination of displacement modes, the higher-order model can also obtain the load of global buckling modes. In order to demonstrate the effectiveness of the proposed method, critical moments of linear and nonlinear under different lengths, web thicknesses, and load heights are calculated.

To evaluate the results, the relative errors between finite element analysis $\left(M_{\mathrm{FEA}}\right)$, Meftah $\left(M_{1}\right)$, present nonlinear $\left(M_{2}\right)$, present linear $\left(M_{3}\right)$, Tan's nonlinear $\left(M_{4}\right)$, and Tan's linear $\left(M_{5}\right)$ are given. The relative errors of moment are shown as follows:

$$
\Delta_{i}=\left|\frac{M_{i}-M_{\mathrm{FEA}}}{M_{\mathrm{FEA}}}\right| \times 100 \% .
$$

Here, the material and geometrical properties are the same as the previous example. In example 1 , it can be seen that when the aspect ratio of cross section is $b / h=3 / 4$, the 
TABLE 2: The percentage differences of the axial displacement $\left(\Delta u_{i}\right)$.

\begin{tabular}{|c|c|c|c|c|c|c|c|c|c|c|c|c|c|c|c|}
\hline \multirow[b]{2}{*}{$L(\mathrm{~m})$} & \multicolumn{3}{|c|}{ I } & \multicolumn{3}{|c|}{ II } & \multicolumn{3}{|c|}{ III } & \multicolumn{3}{|c|}{ IV } & \multicolumn{3}{|c|}{$\mathrm{V}$} \\
\hline & $\begin{array}{l}\Delta u_{1} \\
(\%)\end{array}$ & $\begin{array}{l}\Delta u_{2} \\
(\%)\end{array}$ & $\begin{array}{l}\Delta u_{3} \\
(\%)\end{array}$ & $\begin{array}{l}\Delta u_{1} \\
(\%)\end{array}$ & $\begin{array}{l}\Delta u_{2} \\
(\%)\end{array}$ & $\begin{array}{l}\Delta u_{3} \\
(\%)\end{array}$ & $\begin{array}{l}\Delta u_{1} \\
(\%)\end{array}$ & $\begin{array}{l}\Delta u_{2} \\
(\%)\end{array}$ & $\begin{array}{l}\Delta u_{3} \\
(\%)\end{array}$ & $\begin{array}{l}\Delta u_{1} \\
(\%)\end{array}$ & $\begin{array}{l}\Delta u_{2} \\
(\%)\end{array}$ & $\begin{array}{l}\Delta u_{3} \\
(\%) \\
\end{array}$ & $\begin{array}{l}\Delta u_{1} \\
(\%)\end{array}$ & $\begin{array}{l}\Delta u_{2} \\
(\%)\end{array}$ & $\begin{array}{l}\Delta u_{3} \\
(\%) \\
\end{array}$ \\
\hline 1 & 18.5 & 29.9 & 26.3 & 12.3 & 21.0 & 19.7 & 17.1 & 22.5 & 19.7 & 14.3 & 15.2 & 15.7 & 13.1 & 23.2 & 16.8 \\
\hline 2 & 12.8 & 22.0 & 16.9 & 12.3 & 20.1 & 14.4 & 18.9 & 32.7 & 19.4 & 15.7 & 17.5 & 17.3 & 11.2 & 14.4 & 11.9 \\
\hline 3 & 13.5 & 19.7 & 15.9 & 14.9 & 18.0 & 16.7 & 19.9 & 27.0 & 21.8 & 18.0 & 21.9 & 19.0 & 9.7 & 24.3 & 18.4 \\
\hline 4 & 21.9 & 28.9 & 25.8 & 19.9 & 26.2 & 23.0 & 20.3 & 34.0 & 21.7 & 20.1 & 25.6 & 21.7 & 7.5 & 29.1 & 19.0 \\
\hline 5 & 13.5 & 26.6 & 19.7 & 20.9 & 32.1 & 26.8 & 25.0 & 31.0 & 28.9 & 20.5 & 30.3 & 27.6 & 5.2 & 23.6 & 17.6 \\
\hline 6 & 15.8 & 29.8 & 25.3 & 15.3 & 26.7 & 21.3 & 14.1 & 28.9 & 25.0 & 21.1 & 28.9 & 22.0 & 8.5 & 17.6 & 14.6 \\
\hline 7 & 16.2 & 23.1 & 17.3 & 13.4 & 27.0 & 13.5 & 9.6 & 28.6 & 12.6 & 13.3 & 29.7 & 19.0 & 9.8 & 24.4 & 22.3 \\
\hline 8 & 18.9 & 26.3 & 19.7 & 14.9 & 25.2 & 17.1 & 8.5 & 18.5 & 9.0 & 6.6 & 26.4 & 16.2 & 6.6 & 20.0 & 9.4 \\
\hline
\end{tabular}

TABLE 3: The percentage differences of the vertical displacement $\left(\Delta w_{i}\right)$.

\begin{tabular}{|c|c|c|c|c|c|c|c|c|c|c|c|c|c|c|c|}
\hline \multirow[b]{2}{*}{$L(\mathrm{~m})$} & \multicolumn{3}{|c|}{ I } & \multicolumn{3}{|c|}{ II } & \multicolumn{3}{|c|}{ III } & \multicolumn{3}{|c|}{ IV } & \multicolumn{3}{|c|}{$\mathrm{V}$} \\
\hline & $\begin{array}{l}\Delta w_{1} \\
(\%)\end{array}$ & $\begin{array}{c}\Delta w_{2} \\
(\%)\end{array}$ & $\begin{array}{c}\Delta w_{3} \\
(\%)\end{array}$ & $\begin{array}{l}\Delta w_{1} \\
(\%)\end{array}$ & $\begin{array}{c}\Delta w_{2} \\
(\%)\end{array}$ & $\begin{array}{c}\Delta w_{3} \\
(\%)\end{array}$ & $\begin{array}{c}\Delta w_{1} \\
(\%)\end{array}$ & $\begin{array}{c}\Delta w_{2} \\
(\%)\end{array}$ & $\begin{array}{c}\Delta w_{3} \\
(\%)\end{array}$ & $\begin{array}{c}\Delta w_{1} \\
(\%)\end{array}$ & $\begin{array}{c}\Delta w_{2} \\
(\%)\end{array}$ & $\begin{array}{c}\Delta w_{3} \\
(\%)\end{array}$ & $\begin{array}{l}\Delta w_{1} \\
(\%)\end{array}$ & $\begin{array}{c}\Delta w_{2} \\
(\%)\end{array}$ & $\begin{array}{c}\Delta w_{3} \\
(\%)\end{array}$ \\
\hline 1 & 5.2 & 14.4 & 5.0 & 9.0 & 10.4 & 9.1 & 17.1 & 21.1 & 20.0 & 2.6 & 4.8 & 4.6 & 11.0 & 14.9 & 11.0 \\
\hline 2 & 3.3 & 10.4 & 4.6 & 17.9 & 24.7 & 18.0 & 17.3 & 26.9 & 20.7 & 2.4 & 8.7 & 5.1 & 11.0 & 17.5 & 16.3 \\
\hline 3 & 9.0 & 16.7 & 9.6 & 12.7 & 18.8 & 16.3 & 13.9 & 27.7 & 19.2 & 5.4 & 17.7 & 12.9 & 6.6 & 14.6 & 11.0 \\
\hline 4 & 1.4 & 16.8 & 9.7 & 14.5 & 20.8 & 16.3 & 13.5 & 26.0 & 18.9 & 5.9 & 16.2 & 13.5 & 6.3 & 16.9 & 14.7 \\
\hline 5 & 1.6 & 17.6 & 6.5 & 15.6 & 26.1 & 20.0 & 16.3 & 24.9 & 17.5 & 8.6 & 17.4 & 16.8 & 5.5 & 19.6 & 18.3 \\
\hline 6 & 3.8 & 16.5 & 6.3 & 19.1 & 26.1 & 25.4 & 13.9 & 24.3 & 16.0 & 8.0 & 16.3 & 11.8 & 6.0 & 20.1 & 15.9 \\
\hline 7 & 2.1 & 16.3 & 6.9 & 5.2 & 18.4 & 16.6 & 11.7 & 16.8 & 16.6 & 9.2 & 17.7 & 12.6 & 8.8 & 13.6 & 10.7 \\
\hline 8 & 2.3 & 10.9 & 6.5 & 7.6 & 11.7 & 10.7 & 5.9 & 20.3 & 15.9 & 8.8 & 9.5 & 9.2 & 9.3 & 21.3 & 19.9 \\
\hline
\end{tabular}

TABLe 4: The percentage differences of the transversal displacement $\left(\Delta v_{i}\right)$.

\begin{tabular}{|c|c|c|c|c|c|c|c|c|c|c|c|c|c|c|c|}
\hline \multirow[b]{2}{*}{$L(\mathrm{~m})$} & \multicolumn{3}{|c|}{ I } & \multicolumn{3}{|c|}{ II } & \multicolumn{3}{|c|}{ III } & \multicolumn{3}{|c|}{ IV } & \multicolumn{3}{|c|}{$\mathrm{V}$} \\
\hline & $\begin{array}{l}\Delta v_{1} \\
(\%)\end{array}$ & $\begin{array}{l}\Delta v_{2} \\
(\%)\end{array}$ & $\begin{array}{l}\Delta v_{3} \\
(\%)\end{array}$ & $\begin{array}{l}\Delta v_{1} \\
(\%)\end{array}$ & $\begin{array}{l}\Delta v_{2} \\
(\%)\end{array}$ & $\begin{array}{l}\Delta v_{3} \\
(\%)\end{array}$ & $\begin{array}{l}\Delta v_{1} \\
(\%)\end{array}$ & $\begin{array}{l}\Delta v_{2} \\
(\%)\end{array}$ & $\begin{array}{l}\Delta v_{3} \\
(\%)\end{array}$ & $\begin{array}{l}\Delta v_{1} \\
(\%)\end{array}$ & $\begin{array}{l}\Delta v_{2} \\
(\%)\end{array}$ & $\begin{array}{l}\Delta v_{3} \\
(\%)\end{array}$ & $\begin{array}{l}\Delta v_{1} \\
(\%)\end{array}$ & $\begin{array}{l}\Delta v_{2} \\
(\%)\end{array}$ & $\begin{array}{l}\Delta v_{3} \\
(\%)\end{array}$ \\
\hline 1 & 18.1 & 24.5 & 21.1 & 11.2 & 25.1 & 23.4 & 3.0 & 8.1 & 7.2 & 18.1 & 22.1 & 21.8 & 10.6 & 25.9 & 13.3 \\
\hline 2 & 11.0 & 22.6 & 19.2 & 13.0 & 17.4 & 15.1 & 7.5 & 23.0 & 17.1 & 2.8 & 13.1 & 4.1 & 11.0 & 25.2 & 17.3 \\
\hline 3 & 11.8 & 21.8 & 13.8 & 13.0 & 31.9 & 30.0 & 8.7 & 23.1 & 17.5 & 4.2 & 11.4 & 13.9 & 12.2 & 22.6 & 16.8 \\
\hline 4 & 13.6 & 19.8 & 18.2 & 12.7 & 15.6 & 17.8 & 6.1 & 24.1 & 10.5 & 9.2 & 16.5 & 14.7 & 2.7 & 24.0 & 17.3 \\
\hline 5 & 10.3 & 21.1 & 20.4 & 10.2 & 19.6 & 21.4 & 9.0 & 21.6 & 12.9 & 9.5 & 23.3 & 16.3 & 6.6 & 22.3 & 18.9 \\
\hline 6 & 10.7 & 19.1 & 17.8 & 14.0 & 18.7 & 21.9 & 2.3 & 18.2 & 5.5 & 4.6 & 20.9 & 8.2 & 6.5 & 24.2 & 17.8 \\
\hline 7 & 5.6 & 25.7 & 15.6 & 9.2 & 17.4 & 14.1 & 2.0 & 19.7 & 7.3 & 2.5 & 18.6 & 4.8 & 3.8 & 21.1 & 17.2 \\
\hline 8 & 2.9 & 20.0 & 11.2 & 2.8 & 4.0 & 9.9 & 1.8 & 11.7 & 2.5 & 3.6 & 10.7 & 8.0 & 9.6 & 20.6 & 17.3 \\
\hline
\end{tabular}

comprehensive performance of STBB is the best. Therefore, the parameters are chosen as $h=200 \mathrm{~mm}$, $b=150 \mathrm{~mm}$, and $L=3 \mathrm{~m}, 4 \mathrm{~m}$, and $5 \mathrm{~m}$, respectively. The comparison of buckling moments relative to upper, center, and bottom flange and their difference is shown in Tables 5-7.

It can be seen from the three tables that all of the models have good consistency. The critical moments of the FEA model are almost bigger than those obtained by linear and nonlinear analysis. This is because the FEA model (generally speaking, a membrane shell with restricted movement) is stiffer than theoretical model. In order to verify the correlation of the buckling phenomenon, four rigid transverse webs with different thickness are carried out by the FEA method. The results of FEA analysis confirm the correlation of grid deformation.
From the three tables, it is obvious that the critical moments decrease with the increase of beam length. And at the same length, the critical moments increase with the increase of web thickness. The results show that the influence of length on the critical moments is always greater than that of web thickness. This fact is clear and useful to all theories. Furthermore, it can be obtained that the Meftah, Tan, and present nonlinear numerical calculations of the critical buckling moment are in good agreement with the FEA results. Tan's results and present linear results are more conservative than the nonlinear buckling moment calculated by FEA. It should be noted that the nonlinear solution of Meftah is the smallest, followed by the nonlinear solution of Tan's method. That is to say, the more the nonlinear coupling terms are considered, the higher the calculation accuracy is. It should be noted that Meftah and Tan's solutions 


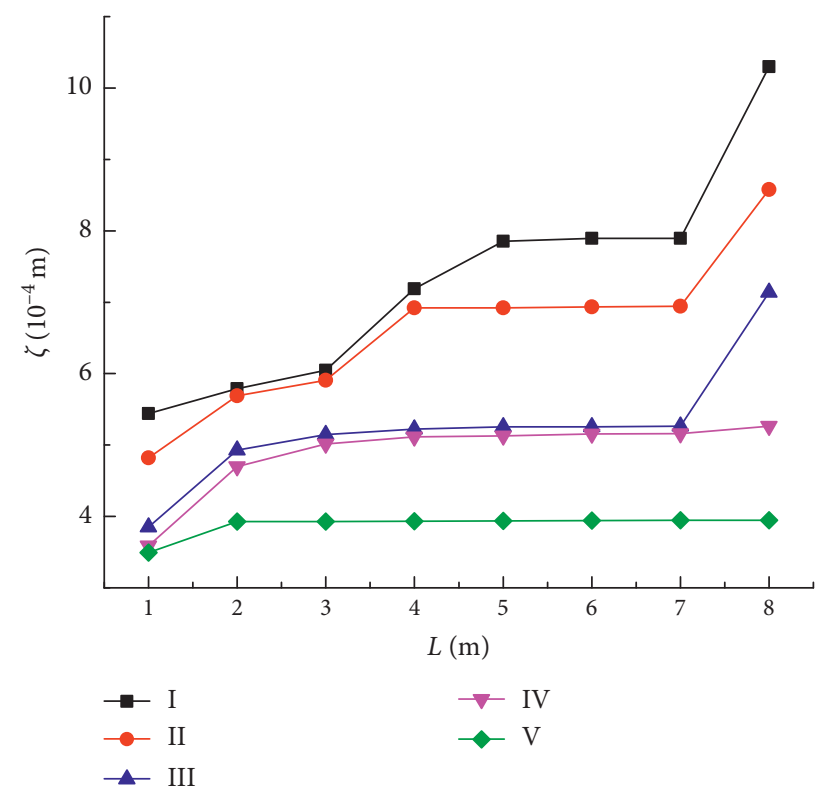

FIGURE 12: Shear lag warping displacement comparison.

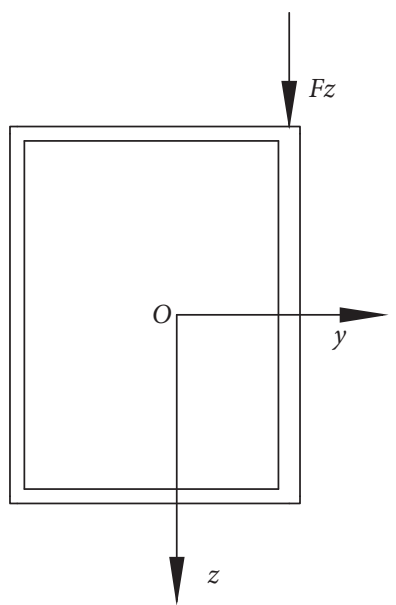

(a)

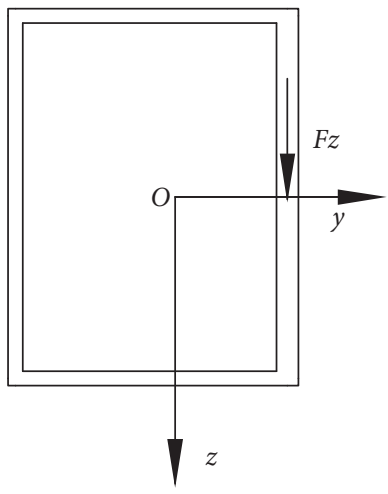

(b)

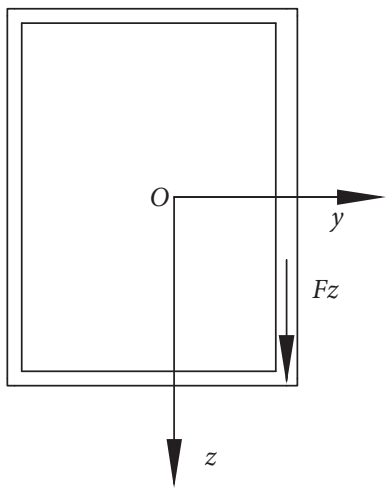

(c)

FIGURE 13: Different heights applied by eccentric loads.

TABLE 5: Buckling moments and relative errors when the load height is $e_{z}=0.1 \mathrm{~m}$.

\begin{tabular}{cccccccccccccc}
\hline $\begin{array}{l}L \\
(\mathrm{~m})\end{array}$ & $\begin{array}{c}t_{2} \\
(\mathrm{~mm})\end{array}$ & $\begin{array}{c}t_{4} \\
(\mathrm{~mm})\end{array}$ & FEA & Meftah & $\begin{array}{c}\text { Buckling moments }(\mathrm{kN} \cdot \mathrm{m}) \\
\text { nonlinear }\end{array}$ & $\begin{array}{c}\text { Present } \\
\text { linear }\end{array}$ & $\begin{array}{c}\text { Tan } \\
\text { nonlinear }\end{array}$ & Tan linear & $\begin{array}{c}\Delta_{1} \\
(\%)\end{array}$ & $\begin{array}{c}\Delta_{2} \\
(\%)\end{array}$ & $\begin{array}{c}\Delta_{3} \\
(\%)\end{array}$ & $\begin{array}{c}\Delta_{4} \\
(\%)\end{array}$ & $\begin{array}{c}\Delta_{5} \\
(\%)\end{array}$ \\
\hline & 5 & 5 & 225667 & 211010.28 & 219611.56 & 209332.79 & 217552.50 & 199428.90 & 6.49 & 2.68 & 7.24 & 3.60 & 11.63 \\
& 8 & 5 & 276309 & 251003.81 & 259641.05 & 251027.69 & 252463.59 & 243544.89 & 9.16 & 6.03 & 9.15 & 8.63 & 11.86 \\
3 & 6.5 & 6.5 & 262569 & 235351.25 & 243006.22 & 234300.30 & 238937.94 & 227981.57 & 10.37 & 7.45 & 10.77 & 9.00 & 13.17 \\
& 8 & 8 & 316051 & 281436.29 & 291725.75 & 279336.82 & 286842.87 & 271458.02 & 10.95 & 7.70 & 6.71 & 9.24 & 14.11 \\
\hline & 5 & 5 & 180955 & 172317.23 & 178798.82 & 168978.27 & 173469.49 & 163869.54 & 4.77 & 1.19 & 6.62 & 4.14 & 9.44 \\
& 8 & 5 & 225538 & 200787.04 & 210657.63 & 198495.28 & 204972.31 & 192801.25 & 10.97 & 6.60 & 11.99 & 9.12 & 14.51 \\
4 & 6.5 & 6.5 & 208127 & 184138.17 & 193421.92 & 181271.11 & 186802.36 & 176519.44 & 11.53 & 7.07 & 12.90 & 10.25 & 15.19 \\
& 8 & 8 & 258906 & 226604.06 & 236073.46 & 224016.84 & 231506.06 & 218561.01 & 12.48 & 8.82 & 13.48 & 10.58 & 15.58 \\
\hline & 5 & 5 & 136689 & 128247.39 & 131239.14 & 128715.02 & 129486.48 & 126401.88 & 6.18 & 3.99 & 8.57 & 5.27 & 7.53 \\
& 8 & 5 & 161808 & 151369.70 & 155009.15 & 150871.19 & 152871.55 & 146421.74 & 6.45 & 4.20 & 6.76 & 5.52 & 9.51 \\
5 & 6.5 & 6.5 & 149180 & 139012.91 & 142197.01 & 138213.96 & 140702.31 & 134633.33 & 6.82 & 4.68 & 7.35 & 5.68 & 9.75 \\
& 8 & 8 & 184686 & 170382.46 & 175750.00 & 169385.73 & 173025.08 & 163285.86 & 7.74 & 4.84 & 8.28 & 6.31 & 11.59 \\
\hline
\end{tabular}


TABLE 6: Buckling moments and relative errors when the load height is $e_{z}=0.0 \mathrm{~m}$.

\begin{tabular}{lccccccccccccc}
\hline $\begin{array}{l}L \\
(\mathrm{~m})\end{array}$ & $\begin{array}{c}t_{2} \\
(\mathrm{~mm})\end{array}$ & $\begin{array}{c}t_{4} \\
(\mathrm{~mm})\end{array}$ & FEA & Meftah & $\begin{array}{c}\text { Buckling moments }(\mathrm{KN} \cdot \mathrm{m}) \\
\text { Present }\end{array}$ & $\begin{array}{c}\text { Present } \\
\text { nonlinear }\end{array}$ & $\begin{array}{c}\text { Tan } \\
\text { nonlinear }\end{array}$ & Tan linear & $\begin{array}{c}\Delta_{1} \\
(\%)\end{array}$ & $\begin{array}{c}\Delta_{2} \\
(\%)\end{array}$ & $\begin{array}{c}\Delta_{3} \\
(\%)\end{array}$ & $\begin{array}{c}\Delta_{4} \\
(\%)\end{array}$ & $\begin{array}{c}\Delta_{5} \\
(\%)\end{array}$ \\
\hline & 5 & 5 & 225835 & 213002.21 & 223166.87 & 211266.27 & 220136.36 & 206738.29 & 5.68 & 1.18 & 6.45 & 2.52 & 8.46 \\
3 & 8 & 5 & 284822 & 259501.48 & 263471.40 & 252847.55 & 261261.62 & 248003.56 & 8.89 & 7.50 & 11.23 & 8.27 & 12.93 \\
& 6.5 & 6.5 & 279215 & 250135.71 & 255005.17 & 246604.78 & 253219.72 & 242447.34 & 10.41 & 8.67 & 11.68 & 9.31 & 13.17 \\
& 8 & 8 & 319554 & 285775.59 & 291819.43 & 282277.02 & 288867.14 & 276512.60 & 10.57 & 8.68 & 11.67 & 9.60 & 13.47 \\
\hline & 5 & 5 & 185347 & 173739.28 & 179231.77 & 170477.61 & 179247.22 & 166251.83 & 6.26 & 3.30 & 8.02 & 3.29 & 10.30 \\
4 & 8 & 5 & 232178 & 214779.69 & 222355.92 & 202710.09 & 217341.62 & 200551.73 & 7.49 & 4.23 & 12.69 & 6.39 & 13.62 \\
& 6.5 & 6.5 & 224293 & 204061.08 & 214146.40 & 194871.77 & 205642.26 & 191828.59 & 9.02 & 4.52 & 13.12 & 8.32 & 14.47 \\
& 8 & 8 & 295826 & 268658.00 & 281732.67 & 256199.83 & 269211.21 & 252482.85 & 9.18 & 4.76 & 13.40 & 9.00 & 14.62 \\
\hline & 5 & 5 & 138531 & 129328.49 & 133717.80 & 128925.13 & 130852.96 & 127093.14 & 6.64 & 3.47 & 6.93 & 5.54 & 8.26 \\
& 8 & 5 & 162876 & 148774.34 & 155911.16 & 147076.83 & 152361.35 & 144382.62 & 8.66 & 4.28 & 9.70 & 6.46 & 11.35 \\
5 & 6.5 & 6.5 & 159572 & 145679.79 & 152418.63 & 143205.59 & 147403.37 & 140670.70 & 8.71 & 4.48 & 10.26 & 7.63 & 11.84 \\
& 8 & 8 & 185976 & 168907.25 & 176252.37 & 165829.93 & 171598.74 & 162866.93 & 9.18 & 5.23 & 10.83 & 7.73 & 12.43 \\
\hline
\end{tabular}

TABLe 7: Buckling moments and relative errors when the load height is $e_{z}=-0.1 \mathrm{~m}$.

\begin{tabular}{cccccccccccccc}
\hline $\begin{array}{l}L \\
(\mathrm{~m})\end{array}$ & $\begin{array}{c}t_{2} \\
(\mathrm{~mm})\end{array}$ & $\begin{array}{c}t_{4} \\
(\mathrm{~mm})\end{array}$ & FEA & Meftah & $\begin{array}{c}\text { B } \\
\text { nonlinear }\end{array}$ & $\begin{array}{c}\text { Present } \\
\text { linear }\end{array}$ & $\begin{array}{c}\text { Tan } \\
\text { nonlinear }\end{array}$ & Tan linear & $\begin{array}{c}\Delta_{1} \\
(\%)\end{array}$ & $\begin{array}{c}\Delta_{2} \\
(\%)\end{array}$ & $\begin{array}{c}\Delta_{3} \\
(\%)\end{array}$ & $\begin{array}{c}\Delta_{4} \\
(\%)\end{array}$ & $\begin{array}{c}\Delta_{5} \\
(\%)\end{array}$ \\
\hline & 5 & 5 & 227568 & 218984.20 & 224733.00 & 213629.25 & 220487.77 & 208314.22 & 3.77 & 1.25 & 6.13 & 3.11 & 8.46 \\
& 8 & 5 & 286538 & 271574.24 & 279651.51 & 267208.35 & 271402.49 & 261601.91 & 5.22 & 2.40 & 6.75 & 5.28 & 8.70 \\
3 & 6.5 & 6.5 & 280801 & 261425.78 & 273238.50 & 261238.57 & 264030.17 & 253839.75 & 6.90 & 2.69 & 6.97 & 5.97 & 9.60 \\
& 8 & 8 & 321619 & 286037.73 & 309914.91 & 285676.66 & 291390.65 & 278627.90 & 11.06 & 3.64 & 11.18 & 9.40 & 13.37 \\
\hline & 5 & 5 & 193686 & 176416.22 & 184194.28 & 175451.18 & 180291.54 & 171150.55 & 8.92 & 4.90 & 9.41 & 6.92 & 11.64 \\
& 8 & 5 & 252012 & 227827.91 & 239620.01 & 225511.64 & 230640.22 & 221674.48 & 9.60 & 4.92 & 10.52 & 8.48 & 12.04 \\
4 & 6.5 & 6.5 & 231983 & 210841.97 & 220204.92 & 206846.99 & 211561.88 & 201911.15 & 9.11 & 5.08 & 10.84 & 8.80 & 12.96 \\
& 8 & 8 & 297230 & 270095.40 & 282007.76 & 263162.24 & 270816.00 & 257204.08 & 9.13 & 5.12 & 11.46 & 8.89 & 13.47 \\
\hline & 5 & 5 & 138719 & 129404.94 & 136127.97 & 130180.71 & 134545.10 & 129216.81 & 6.71 & 1.87 & 6.16 & 3.01 & 6.85 \\
& 8 & 5 & 163506 & 151520.11 & 157885.73 & 149802.14 & 154473.85 & 144995.82 & 7.33 & 3.44 & 8.38 & 5.52 & 11.32 \\
5 & 6.5 & 6.5 & 160061 & 147786.97 & 152596.97 & 143508.83 & 150411.54 & 140978.89 & 7.67 & 4.66 & 10.34 & 6.03 & 11.92 \\
& 8 & 8 & 186072 & 170100.49 & 177063.44 & 166817.47 & 174786.55 & 163819.47 & 8.58 & 4.84 & 10.35 & 6.07 & 11.96 \\
\hline
\end{tabular}

under the same conditions are lower than those of the present approach; this is due to the generalized eigenvectors of the classical models and the initial assumptions-essentially without taking the shear deformation into account, which is very important for the different thicknesses of the web model. The nonlinear critical moments obtained by present method is closest to those obtained by FEA. And the linear solution of present method is larger than the linear solution of Tan's method; it can be seen that considering the effect of shear lag effect can more comprehensively supplement the mechanical properties of STBB. The difference between linear and nonlinear relative error is very impressive. The linear and nonlinear relative errors of Tan and the present method can reach about $8 \%$ and $8.63 \%$ at the maximum, respectively. From the relative errors $\Delta_{1}, \Delta_{2}$, and $\Delta_{4}$, it can be seen that the numerical and analytical solutions are consistent and highly nonlinear. The relative error $\Delta_{2}$ is almost the smallest value, while $\Delta_{5}$ is the biggest one. In this case, by comparing the moments of STBB before and after buckling, it can be seen that only the nonlinear solution is more accurate.
By comparing the three tables, it can be seen that when the load is applied to the top plane of STBB, the bending moment is the smallest. And when the load is applied to the bottom plane, the beam resistance to lateral buckling is the greatest. Therefore, we can think that the lateral buckling strength depends on the load height parameter. In the postbuckling state, the shear lag effect term is added to maximize the resistance of STBB to lateral buckling. In general, as the web becomes thicker, the relative error becomes larger overall. Regardless of beam length and the location where the concentrated load was applied, $M_{\mathrm{cr}}$ values for nonuniform web thickness $\left(t_{1}=5 \mathrm{~mm}\right.$ and $\left.t_{2}=8 \mathrm{~mm}\right)$ are higher than those for the same web thickness $\left(t_{1}=t_{2}=6.5 \mathrm{~mm}\right)$. With the increase of the length, the difference of the critical moments (for both uniform and unequal webs) becomes more obvious, and the unequal web of beams show better mechanical properties. Buckling moment is mainly affected by the web resistance. It is clear that the phenomenon is due solely to coupling-induced geometrical nonlinear effect. 


\section{Conclusion}

A new kinematics model is used to analyze the stability of straight thin-walled box beam (STBB). Based on the conventional motion variables, the shear lag modes are added to obtain that the beam section contains six modes of extension, bending, torsion, distortion, and shear lag under the action of shear deformation. The analytical formulas take the deformation, load height, and unequal web thickness into account under combined axial and vertical loads. In this theory, six sets of coupled equilibrium differential equations are derived to describe the longitudinal and transversal deformation through six displacement fields. In particular, when the straight thin-walled box beam is subjected to combined axial and vertical loads, these formulas are naturally applicable to capture bending, torsional, distortion, and shear lag responses.

Present theory puts forward a theoretical basis for studying the mechanical properties of STBB and the ways to be affected by adding shear lag effect. Unlike traditional Meftah and Tan's theories, present theory shows that the beam response is largely dependent on the shear lag. Various numerical results are compared with those of Meftah, Tan, and shell finite element (Ansys).

With regard to the different cross sections and lengths, the following conclusions are given:

(i) When the cross section ratio is greater than 1 , the geometric variation of cross section has little influence on the axial length.

(ii) The shear lag displacement is conducive to the prediction of longitudinal displacement.

(iii) For the long beam, the shear lag warping displacement can reflect the vertical displacement more accurately.

(iv) Generalized shear lag deformation increases the predicted torsional stiffness of the beam. (v) The distortion under the coupling effect of the six modes (adding shear lag effect) is more conservative than that of the five modes.

(vi) The shear lag warping displacement increases with the increase of cross section ratio $(b / h)$.

For the different load positions and the thicknesses of webs, the following results are reached:

(i) The nonlinear critical moments of present method are closest to those of FEA.

(ii) The relative error $\Delta_{2}$ is almost the smallest value, while $\Delta_{5}$ is the biggest one.

(iii) The lateral buckling strength depends on the load height parameter.

(iv) $M_{c r}$ values for nonuniform web thickness $\left(t_{1}=5 \mathrm{~mm}\right.$ and $t_{2}=8 \mathrm{~mm}$ ) are higher than those for the same web thickness $\left(t_{1}=t_{2}=6.5 \mathrm{~mm}\right)$.

The effect of shear lag can more comprehensively supplement the mechanical properties of STBB, and the nonlinear solution is more accurate. In the postbuckling state, the shear lag effect term is added to maximize the resistance of STBB to lateral buckling.

The calculation results are in good agreement with the results of finite element and other theoretical analysis. The above observation results show that the geometry has a great influence on the nonlinear buckling of STBB. For these reasons, further development of this research should focus on the relationship between the material properties of beams and the modes. The developed analytical method is sufficiently efficient and accurate.

\section{Appendix}

\section{A. Detailed Expressions of These Forces and Torques}

$$
\left.\begin{array}{c}
N=E\left[\begin{array}{c}
\sum_{i=4} F_{i} u_{0}^{\prime}(x)+\frac{1}{2} \sum_{i=4} F_{i}\left[u_{0}^{\prime}(x)^{2}+v_{0}^{\prime}(x)^{2}+w_{0}^{\prime}(x)^{2}\right]+\frac{1}{2}\left(\sum_{i=1,3} A_{2}^{i}+\sum_{i=2,4} B_{2}^{i}+\sum_{i=1,3} A_{5}^{i}+\sum_{i=2,4} B_{5}^{i}\right) \theta(x)^{2}-\sum_{i=1,3} A_{6}^{i} v_{0}^{\prime \prime}(x) \chi_{0}^{\prime \prime}(x) \\
\left.+\sum_{i=1,3} A_{19}^{i}+\sum_{i=2,4} B_{19}^{i}+\sum_{i=1,3} A_{20}^{i}+\sum_{i=2,4} B_{20}^{i}\right) \chi^{\prime}(x)^{2}+\frac{1}{2} \sum_{i=1,3} A_{36}^{i} \zeta_{z}^{\prime}(x)^{2}-\left(\sum_{i=1,3} A_{21}^{i}+\sum_{i=2,4} B_{21}^{i}\right) \theta^{\prime}(x) \chi^{\prime}(x)-\sum_{i=2,4} B_{6}^{i} w_{0}^{\prime \prime}(x) \chi_{0}^{\prime \prime}(x)
\end{array}\right], \\
-\sum_{i=1,3} A_{37}^{i} \zeta_{z}^{\prime}(x) w_{0}^{\prime \prime}(x)+\frac{1}{2}\left(\sum_{i=1,3} A_{2}^{i}+\sum_{i=2,4} B_{5}^{i}\right) w_{0}^{\prime \prime}(x)^{2}+\frac{1}{2}\left(\sum_{i=1,3} A_{5}^{i}+\sum_{i=2,4} B_{2}^{i}\right) v_{0}^{\prime \prime}(x)^{2}+\frac{1}{2}\left(\sum_{i=1,3} A_{7}^{i}+\sum_{i=2,4} B_{7}^{i}\right)\left[\theta_{0}^{\prime \prime}(x)^{2}+\chi_{0}^{\prime}(x)^{2}\right]
\end{array}\right]
$$




$$
\begin{aligned}
& M_{z}=E\left[\begin{array}{c}
\left(\sum_{i=1,3} A_{5}^{i}+\sum_{i=2,4} B_{2}^{i}\right)\left[v_{0}^{\prime \prime}(x)+u_{0}^{\prime}(x) v_{0}^{\prime \prime}\right]-\frac{1}{4} \sum_{i=1,3}\left[A_{38}^{i} \zeta_{z}^{\prime}(x)+\theta_{0}^{\prime \prime}(x) \zeta_{z}^{\prime}(x)\right]+\left(\sum_{i=1,3} A_{7}^{i}-\sum_{i=2,4} B_{7}^{i}\right) w_{0}^{\prime \prime}(x) \theta_{0}^{\prime \prime}(x) \\
+\left(\sum_{i=2,4} B_{2}^{i}+\sum_{i=1,3} A_{5}^{i}\right) w_{0}^{\prime}(x) \theta^{\prime}(x)-\sum_{i=2,4} B_{11}^{i} v_{0}^{\prime}(x) \chi^{\prime}(x)-\left(\sum_{i=1,3} A_{11}^{i}+\sum_{i=2,4} B_{2}^{i}\right) w_{0}^{\prime}(x) \chi^{\prime}(x)-\sum_{i=2,4} A_{6}^{i}\left[\chi_{0}^{\prime \prime}(x)+u_{0}^{\prime}(x) \chi_{0}^{\prime \prime}(x)\right]
\end{array}\right], \\
& M_{\psi_{z}}=E\left[\begin{array}{c}
\left(\sum_{i=2,4} B_{2}^{i}-\sum_{i=1,3} A_{13}^{i}\right) v_{0}^{\prime \prime}(x)-\sum_{i=1,3} A_{11}^{i}\left(w_{0}^{\prime \prime}(x)+u_{0}^{\prime}(x) w_{0}^{\prime \prime}(x)\right)-\sum_{i=1,3} A_{14}^{i}\left(\chi_{z}^{\prime \prime}(x)+u_{0}^{\prime}(x) \chi_{z}^{\prime \prime}(x)\right) \\
+\sum_{i=1,3} A_{13}^{i} u_{0}^{\prime \prime}(x) v_{0}^{\prime \prime}(x)-\sum_{i=1,3} A_{39}^{i} \theta^{\prime \prime}(x) \zeta_{2}^{\prime}(x)-\sum_{i=2,4} B_{7}^{i} w_{0}^{\prime \prime}(x) \theta^{\prime \prime}(x)-\sum_{i=1,3} B_{1}^{i} \zeta_{2}^{\prime}(x) v_{0}^{\prime \prime}(x)-\sum_{i=1,3} B_{8}^{i} \theta^{\prime \prime}(x) \chi^{\prime \prime}(x)
\end{array}\right], \\
& M_{\psi_{y}}=E\left[\begin{array}{c}
\left(\sum_{i=1,3} A_{2}^{i}-\sum_{i=2,4} B_{13}^{i}\right) w_{0}^{\prime \prime}(x)-\sum_{i=2,4} B_{14}^{i} \chi^{\prime \prime}(x)-\sum_{i=1,3} A_{37}^{i}\left(\zeta_{z}^{\prime}(x)+u_{0}^{\prime}(x) \zeta_{z}^{\prime}(x)\right) \\
\sum_{i=1,3} A_{7}^{i} v_{0}^{\prime \prime}(x) \theta^{\prime \prime}(x)+\left(\sum_{i=1,3} A_{8}^{i}+\sum_{i=2,4} B_{8}^{i}\right) \theta^{\prime \prime}(x) \chi^{\prime \prime}(x)
\end{array}\right], \\
& M_{R}=E\left[\begin{array}{c}
\left(\sum_{i=1,3} A_{2}^{i}+\sum_{i=2,4} B_{2}^{i}+\sum_{i=1,3} A_{5}^{i}+\sum_{i=2,4} B_{5}^{i}\right)\left[u_{0}^{\prime}(x)+\frac{1}{2}\left(u_{0}^{\prime}(x)^{2}+v_{0}^{\prime}(x)^{2}+u_{0}^{\prime}(x)^{2}\right)\right]+\frac{1}{2}\left(\sum_{i=1,3} A_{28}^{i}+\sum_{i=2,4} B_{28}^{i}\right) \theta(x)^{2} \\
+\frac{1}{2}\left(\sum_{i=1,3} A_{29}^{i}+\sum_{i=2,4} B_{29}^{i}\right) \chi^{\prime}(x)^{2}+\frac{1}{2}\left(\frac{5}{2} \sum_{i=1,3} A_{36}^{i}+\sum_{i=1,3} A_{45}^{i}\right) \zeta_{z}^{\prime}(x)^{2}-\left(\sum_{i=1,3} A_{30}^{i}+\sum_{i=2,4} B_{30}^{i}\right) \theta^{\prime}(x) \chi^{2}(x) \\
+\frac{1}{2}\left(\sum_{i=1,3} A_{7}^{i}+\sum_{i=2,4} B_{7}^{i}+\sum_{i=1,3} A_{4}^{i}+\sum_{i=2,4} B_{32}^{i}\right) w_{0}^{\prime \prime}(x)^{2}+\frac{1}{2}\left(\sum_{i=1,3} A_{7}^{i}+\sum_{i=2,4} B_{7}^{i}+\sum_{i=1,3} A_{32}^{i}+\sum_{i=2,4} B_{4}^{i}\right) v_{0}^{\prime \prime}(x)^{2} \\
+\frac{1}{2}\left(\sum_{i=1,3} A_{8}^{i}+\sum_{i=2,4} B_{8}^{i}+\sum_{i=1,3} A_{9}^{i}+\sum_{i=2,4} B_{9}^{i}\right)\left[\theta^{\prime \prime}(x)^{2}+\chi^{\prime \prime}(x)^{2}\right]-\sum_{i=1,3} A_{46}^{i} w_{0}^{\prime \prime}(x) \zeta_{z}^{\prime}(x)
\end{array}\right], \\
& \left(\sum_{i=1,3} A_{19}^{i}+\sum_{i=2,4} B_{19}^{i}+\sum_{i=1,3} A_{20}^{i}+\sum_{i=2,4} B_{20}^{i}\right)\left[u_{0}^{\prime}(x)+\frac{1}{2}\left(u_{0}^{\prime}(x)^{2}+v_{0}^{\prime}(x)^{2}+w_{0}^{\prime}(x)^{2}\right)\right]+\frac{1}{2} \sum_{i=1,3} A_{47}^{i} \zeta_{z}^{\prime}(x)^{2} \\
& -\left(\sum_{i=1,3} A_{41}^{i}+\sum_{i=2,4} B_{41}^{i}\right)\left[\theta^{\prime \prime}(x)+u_{0}^{\prime}(x) \theta^{\prime \prime}(x)\right]-\sum_{i=1,3} A_{48}^{i} w_{0}^{\prime \prime}(x) \zeta_{z}^{\prime}(x) \zeta_{z}^{\prime}(x)-\sum_{i=1,3} A_{49}^{i} v_{0}^{\prime \prime}(x) \zeta_{z}^{\prime}(x) \zeta_{z}^{\prime}(x) \\
& M_{\psi}=E \quad+\frac{1}{2}\left(\sum_{i=1,3} A_{29}^{i}+\sum_{i=2,4} B_{29}^{i}\right) \theta^{\prime}(x)^{2}+\frac{1}{2}\left(\sum_{i=1,3} A_{42}^{i}+\sum_{i=2,4} B_{42}^{i}\right) \chi^{\prime}(x)^{2}-\left(\sum_{i=1,3} A_{17}^{i}+\sum_{i=2,4} B_{17}^{i}\right) \theta^{\prime}(x) \chi^{\prime}(x) \\
& +\frac{1}{2}\left(\sum_{i=1,3} A_{43}^{i}+\sum_{i=2,4} B_{44}^{i}\right) w_{0}^{\prime \prime}(x)^{2}+\frac{1}{2}\left(\sum_{i=1,3} A_{44}^{i}+\sum_{i=2,4} B_{43}^{i}\right) v_{0}^{\prime \prime}(x)^{2}+\frac{1}{2}\left(\sum_{i=1,3} A_{52}^{i}+\sum_{i=2,4} B_{52}^{i}\right)\left[\theta^{\prime \prime}(x)^{2}+\chi^{\prime \prime}(x)^{2}\right] \\
& \left.+\left(\sum_{i=2,4} B_{41}^{i}-\sum_{i=1,3} A_{41}^{i}\right) v_{0}^{\prime \prime} w_{0}^{\prime \prime}(x)+\left(\sum_{i=2,4} B_{52}^{i}-\sum_{i=1,3} A_{53}^{i}\right) w_{0}^{\prime \prime}(x) \chi^{\prime \prime}(x)+\left(\sum_{i=1,3} A_{52}^{i}+\sum_{i=2,4} B_{53}^{i}\right) v_{0}^{\prime \prime}(x) \chi^{\prime \prime}(x)\right] \\
& \left(\sum_{i=1,3} A_{21}^{i}+\sum_{i=2,4} B_{21}^{i}\right)\left[u_{0}^{\prime}(x)+\frac{1}{2}\left(u_{0}^{\prime}(x)^{2} v_{0}^{\prime}(x)^{2}+w_{0}^{\prime}(x)^{2}\right)\right]+\frac{1}{2}\left(\sum_{i=1,3} A_{30}^{i}+\sum_{i=2,4} B_{30}^{i}\right) \theta \boldsymbol{r}(x)^{2}-\sum_{i=1,3} A_{58}^{i} v_{0}^{\prime \prime}(x) \zeta_{z}^{\prime}(x) \\
& +\frac{1}{2}\left(\sum_{i=1,3} A_{17}^{i}+\sum_{i=2,4} B_{17}^{i}\right) \chi^{\prime}(x)^{2}+\frac{1}{2} \sum_{i=1,3} A_{50}^{i} \zeta_{z}^{\prime}(x)^{2}-\left(\sum_{i=1,3} A_{54}^{i}+\sum_{i=2,4} B_{54}^{i}\right) \theta \prime(x) \chi \prime(x)-\sum_{i=1,3} A_{51}^{i} w_{0}^{\prime \prime}(x) \zeta_{z}^{\prime}(x) \\
& M_{\psi R}=E \\
& +\sum_{i=1,3} A_{59}^{i} \chi_{0}^{\prime \prime}(x) \zeta_{z}^{\prime}+\frac{1}{2}\left(\sum_{i=1,3} A_{4}^{i}-\sum_{i=1,3} A_{15}^{i}+\sum_{i=2,4} B_{55}^{i}\right) w_{0}^{\prime \prime}(x)^{2}+\frac{1}{2}\left(\sum_{i=1,3} A_{55}^{i}+\sum_{i=1,3} B_{4}^{i}-\sum_{i=2,4} B_{15}^{i}\right) v_{0}^{\prime \prime}(x)^{2} \\
& {\left[+\frac{1}{2}\left(\sum_{i=1,3} A_{56}^{i}+\sum_{i=2,4} B_{56}^{i}\right)\left[\theta^{\prime \prime}(x)^{2}+\chi^{\prime \prime}(x)^{2}\right]+\left(\sum_{i=1,3} A_{57}^{i}+2 \sum_{i=2,4} B_{18}^{i}\right) v_{0}^{\prime \prime}(x) w_{0}^{\prime \prime}(x)+\left(2 \sum_{i=1,3} A_{18}^{i}+\sum_{i=2,4} B_{57}^{i}\right) w_{0}^{\prime \prime}(x) \chi^{\prime \prime}(x)\right]}
\end{aligned}
$$




$$
\begin{aligned}
& B_{\omega_{a}}=E\left[\begin{array}{c}
\frac{1}{2}\left(\sum_{i=1,3} A_{41}^{i}+\sum_{i=2,4} B_{41}^{i}\right) \theta^{\prime}(x)^{2}-\left(\sum_{i=1,3} A_{7}^{i}+\sum_{i=2,4} B_{7}^{i}\right)\left[\theta^{\prime \prime}(x)+u_{0}^{\prime}(x) \theta^{\prime \prime}(x)\right]+\sum_{i=1,3} A_{60}^{i} \chi_{0}^{\prime \prime}(x) \zeta^{\prime}(x) \\
+\left(\sum_{i=2,4} B_{6}^{i}-\sum_{i=1,3} A_{6}^{i}\right) v_{0}^{\prime \prime}(x) w_{0}^{\prime \prime}(x)+\sum_{i=1,3} B_{8}^{i} v_{0}^{\prime \prime}(x) \chi_{0}^{\prime \prime}(x)-\sum_{i=1,3} A_{38}^{i} v_{0}^{\prime \prime}(x) \zeta_{z}^{\prime}(x)
\end{array}\right], \\
& B_{\omega_{d}}=E\left[\sum_{i=1,3} A_{60}^{i} \theta^{\prime \prime}(x) \zeta_{z}^{\prime}(x)-\left(\sum_{i=1,3} A_{7}^{i}+\sum_{i=2,4} B_{7}^{i}\right)\left[\chi_{0}^{\prime \prime}(x)+u_{0}^{\prime}(x) \chi_{0}^{\prime \prime}(x)\right]-\sum_{i=1,3} A_{6}^{i}\left[v_{0}^{\prime}(x) \theta r(x)+w_{0}^{\prime}(x) \theta \prime(x)\right]-\sum_{i=1,3} B_{8}^{i} v^{\prime \prime}{ }_{0}(x) \theta^{\prime \prime}(x)\right], \\
& M_{\omega_{\zeta_{2}}^{2}}=E\left[\begin{array}{l}
\sum_{i=1,3} A_{36}^{i}\left[u_{0}^{\prime}(x)+\frac{1}{2}\left(u_{0}^{\prime}(x)^{2}+v_{0}^{\prime}(x)^{2}+w_{0}^{\prime}(x)^{2}\right)\right]+\frac{1}{2}\left[2 \sum_{i=1,3} A_{36}^{i}+\sum_{i=1,3} A_{45}^{i}\right] \theta_{0}^{\prime}(x)^{2}+\frac{1}{2} \sum_{i=1,3} A_{47}^{i} \chi_{0}^{\prime}(x)^{2}+\sum_{i=1,3} A_{36}^{i} w_{0}^{\prime \prime}(x)^{2} \\
-\sum_{i=1,3} A_{50}^{i} \theta_{0}^{\prime}(x) \chi_{0}^{\prime}(x)-\sum_{i=1,3} A_{37}^{i} \chi^{\prime \prime}(x) \zeta_{z}^{\prime}(x)+\frac{1}{2} \sum_{i=1,3} A_{45}^{i} v_{0}^{\prime \prime}(x)^{2}+\frac{6}{7} \sum_{i=1,3} A_{60}^{i} v_{0}^{\prime \prime}(x) \chi^{\prime \prime}(x)+\frac{1}{2} \sum_{i=1,3} A_{62}^{i}\left[\theta_{0}^{\prime \prime}(x)^{2}+\chi_{0}^{\prime \prime}(x)^{2}\right]
\end{array}\right], \\
& M_{z \omega_{\zeta_{z}}}=E\left[\begin{array}{c}
\sum_{i=1,3} A_{37}^{i}\left[u_{0}^{\prime}(x)+\frac{1}{2}\left(u_{0}^{\prime}(x)^{2}+v_{0}^{\prime}(x)^{2}+w_{0}^{\prime}(x)^{2}\right)\right]+\frac{1}{2} \sum_{i=1,3} A_{46}^{i} \theta_{0}^{\prime}(x)^{2}+\frac{1}{2} \sum_{i=1,3} A_{48}^{i} \chi_{0}^{\prime}(x)^{2} \\
-\sum_{i=1,3} A_{51}^{i} \theta_{0}^{\prime}(x) \chi_{0}^{\prime}(x)+\frac{1}{2} \sum_{i=1,3} A_{62}^{i} w_{0}^{\prime \prime}(x)^{2}+\frac{1}{8} \sum_{i=1,3} B_{38}^{i} v_{0}^{\prime \prime}(x)^{2}+\frac{7}{12} \sum_{i=1,3} A_{62}^{i}\left[\theta_{0}^{\prime \prime}(x)^{2}+\chi_{0}^{\prime \prime}(x)^{20}\right] \\
-\sum_{i=1,3} A_{60}^{i}\left[v_{0}^{\prime \prime}(x) \theta_{0}^{\prime \prime}(x) v_{0}^{\prime \prime}(x) \chi_{0}^{\prime \prime}(x)\right]-\frac{7}{6} \sum_{i=1,3} A_{62}^{i} \theta_{0}^{\prime \prime}(x) \chi_{0}^{\prime \prime}(x)-2 \sum_{i=1,3} A_{36}^{i} w_{0}^{\prime \prime}(x) \zeta_{z}^{\prime}(x)
\end{array}\right], \\
& M_{\omega_{a} \omega_{\zeta_{z}}}=E\left[\sum_{i=1,3} A_{39}^{i} w_{0}^{\prime}(x) \chi_{0}^{\prime}(x)-\sum_{i=1,3} A_{60}^{i}\left[\chi_{0}^{\prime \prime}(x)+u_{0}^{\prime}(x) \chi_{0}^{\prime \prime}(x)-\theta_{0}^{\prime \prime}(x) \zeta_{z}^{\prime}(x)\right]+\frac{1}{4} \sum_{i=1,3} A_{38}^{i}\left[v_{0}^{\prime \prime}(x)+u_{0}^{\prime}(x) v_{0}^{\prime \prime}(x)\right]\right], \\
& M_{\omega_{d} \omega_{\zeta_{z}}}=E\left[\begin{array}{c}
\frac{1}{4} \sum_{i=1,3} A_{38}^{i} w_{0}^{\prime}(x) \theta_{0}^{\prime}(x)-\sum_{i=1,3} A_{59}^{i} \theta_{0}^{\prime}(x) \chi_{0}^{\prime}(x)+\frac{1}{2} \sum_{i=1,3} A_{49}^{i} \chi_{0}^{\prime}(x)^{2}+\frac{6}{7} \sum_{i=1,3} A_{60}^{i} v_{0}^{\prime \prime}(x) \zeta_{z}^{\prime}(x) \\
-\sum_{i=1,3} A_{60}^{i}\left[\theta_{0}^{\prime \prime}(x)+u_{0}^{\prime}(x) \theta_{0}^{\prime \prime}(x)+\chi_{0}^{\prime \prime}(x) \zeta_{z}^{\prime}(x)+v_{0}^{\prime \prime}(x) w_{0}^{\prime \prime}(x)\right]
\end{array}\right], \\
& M_{z^{2}}=E\left[\begin{array}{c}
\left(\sum_{i=1,3} A_{2}^{i}+\sum_{i=2,4} B_{5}^{i}\right)\left[u_{0}^{\prime}(x)+\frac{1}{2}\left(u_{0}^{\prime}(x)^{2}+v_{0}^{\prime}(x)^{2}+w_{0}^{\prime}(x)^{2}\right)\right]+\sum_{i=1,3} A_{36}^{i} \zeta_{z}^{\prime}(x)^{2}+\frac{1}{2}\left(\sum_{i=1,3} A_{43}^{i}+\sum_{i=2,4} B_{44}^{i}\right) \chi^{\prime}(x)^{2} \\
+\frac{1}{2}\left(\sum_{i=1,3} A_{7}^{i}+\sum_{i=2,4} B_{7}^{i}+\sum_{i=1,3} A_{4}^{i}+\sum_{i=2,4} B_{32}^{i}\right) \theta^{\prime}(x)^{2}-\left(\sum_{i=1,3} A_{4}^{i}-\sum_{i=1,3} A_{15}^{i}+\sum_{i=2,4} B_{55}^{i}\right) \theta^{\prime}(x) \chi^{\prime}(x)-\sum_{i=1,3} A_{4}^{i} w_{0}^{\prime}(x) \zeta_{z}^{\prime}(x) \\
+\frac{1}{2}\left(\sum_{i=1,3} A_{7}^{i}+\sum_{i=2,4} B_{7}^{i}\right) v_{0}^{\prime \prime}(x)^{2}+\frac{1}{2}\left(\sum_{i=1,3} A_{9}^{i}+\sum_{i=2,4} B_{33}^{i}\right)\left[\theta^{\prime \prime}(x)^{2}+\chi^{\prime \prime}(x)^{2}\right]+\frac{1}{2}\left(\sum_{i=1,3} A_{4}^{i}+\sum_{i=2,4} B_{732}^{i}\right) w_{0}^{\prime \prime}(x)^{2} \\
-\frac{5}{2} \sum_{i=1,3} A_{82}^{i} w_{0}^{\prime \prime}(x) \zeta_{z}^{\prime}(x)
\end{array}\right],
\end{aligned}
$$




$$
\begin{aligned}
& M_{Y^{2}}=E\left[\begin{array}{c}
\left(\sum_{i=1,3} A_{5}^{i}+\sum_{i=2,4} B_{2}^{i}\right)\left[u_{0}^{\prime}(x)+\frac{1}{2}\left(u_{0}^{\prime}(x)^{2}+v_{0}^{\prime}(x)^{2}+w_{0}^{\prime}(x)^{2}\right)\right]+\frac{1}{2} \sum_{i=1,3} A_{45}^{i} \zeta_{z}^{\prime}(x)^{2}+\frac{1}{2}\left(\sum_{i=1,3} A_{44}^{i}+\sum_{i=2,4} B_{43}^{i}\right) \chi^{\prime}(x)^{2} \\
+\frac{1}{2}\left(\sum_{i=1,3} A_{7}^{i}+\sum_{i=2,4} B_{7}^{i}+\sum_{i=1,3} A_{32}^{i}+\sum_{i=2,4} B_{4}^{i}\right) \theta \prime(x)^{2}-\frac{1}{4} \sum_{i=1,3} A_{5}^{i} w_{0}^{\prime}(x) \zeta_{z}^{\prime}(x) \\
+\frac{1}{2}\left(\sum_{i=1,3} A_{32}^{i}+\sum_{i=2,4} B_{4}^{i}\right) v_{0}^{\prime \prime}(x)^{2}+\frac{1}{2}\left(\sum_{i=1,3} A_{33}^{i}+\sum_{i=2,4} B_{9}^{i}\right)\left[\theta^{\prime \prime}(x)^{2}+\chi^{\prime \prime}(x)^{2}\right]+\frac{1}{2}\left(\sum_{i=1,3} A_{7}^{i}+\sum_{i=2,4} B_{7}^{i}\right) w_{0}^{\prime \prime}(x)^{2} \\
+\sum_{i=1,3} A_{64}^{i} v_{0}^{\prime \prime}(x) \chi^{\prime \prime}(x)-\left(\sum_{i=1,3} A_{55}^{i}+\sum_{i=2,4} B_{4}^{i}-\sum_{i=2,4} B_{15}^{i}\right) \theta \prime(x) \chi^{\prime}(x)
\end{array}\right], \\
& M_{\omega_{a}^{2}}=E\left[\begin{array}{c}
\left(\sum_{i=1,3} A_{7}^{i}+\sum_{i=2,4} B_{7}^{i}\right)\left[u_{0}^{\prime}(x)+\frac{1}{2}\left(u_{0}^{\prime}(x)^{\prime} v_{0}(x)^{2}+w_{0}(x)^{2}\right)\right]+\frac{1}{2}\left(\sum_{i=1,3} A_{62}^{i} \zeta_{z}^{\prime}(x)^{2}+\frac{1}{2}\left(\sum_{i=1,3} A_{52}^{i}+\sum_{i=2,4} B_{52}^{i}\right)\right) \\
+\frac{1}{2}\left(\sum_{i=1,3} A_{8}^{i}+\sum_{i=2,4} B_{8}^{i}+\sum_{i=1,3} A_{9}^{i}+\sum_{i=2,4} B_{9}^{i}\right) \theta \prime(x)^{2}+\left(\sum_{i=1,3} A_{56}^{i}+\sum_{i=2,4} B_{56}^{i}\right) \theta \prime(x) \chi^{\prime}(x)-\frac{7}{6} \sum_{i=1,3} A_{62}^{i} w_{0}^{\prime \prime}(x) \zeta_{z}^{\prime}(x)^{2} \\
+\frac{1}{2}\left(\sum_{i=1,3} A_{33}^{i}+\sum_{i=2,4} B_{9}^{i}\right) v_{0}^{\prime \prime}(x)^{2}+\frac{1}{2}\left(\sum_{i=1,3} A_{35}^{i}+\sum_{i=2,4} B_{35}^{i}\right)\left[\theta^{\prime \prime}(x)^{2} \chi^{\prime \prime}(x)^{2}\right]+\frac{1}{2}\left(\sum_{i=1,3} A_{9}^{i}+\sum_{i=2,4} B_{33}^{i}\right) w_{0}^{\prime \prime}(x)^{2} \\
-\sum_{i=1,3} A_{34}^{i}\left[v_{0}^{\prime \prime}(x) \theta^{\prime \prime}(x)+v_{0}^{\prime \prime}(x) \chi^{\prime \prime}(x)\right]+\sum_{i=1,3} B_{34}^{i} w_{0}^{\prime \prime}(x)^{2} \chi^{\prime \prime}(x)
\end{array}\right], \\
& {\left[\left(\sum_{i=1,3} A_{7}^{i}+\sum_{i=2,4} B_{7}^{i}\right)\left[u_{0}^{\prime}(x)+\frac{1}{2}\left(u_{0}^{\prime}(x)^{2}+v_{0}^{\prime}(x)^{2}+w_{0}^{\prime}(x)^{2}\right)\right]+\frac{1}{2} \sum_{i=1,3} A_{62}^{i} \zeta_{z}(x)^{2}+\frac{1}{2}\left(\sum_{i=1,3} A_{52}^{i}+\sum_{i=2,4} B_{52}^{i}\right) \chi^{\prime}(x)^{2}\right.} \\
& M_{\omega_{d}^{2}}=E+\frac{1}{2}\left(\sum_{i=1,3} A_{8}^{i}+\sum_{i=2,4} B_{8}^{i}+\sum_{i=1,3} A_{9}^{i}+\sum_{i=2,4} B_{9}^{i}\right) \theta^{\prime}(x)^{2}-\frac{7}{6} \sum_{i=1,3} A_{62}^{i} w_{0}^{\prime \prime}(x) \zeta_{z}^{\prime}(x)+\frac{1}{2}\left(\sum_{i=1,3} A_{33}^{i}+\sum_{i=2,4} B_{9}^{i}\right) v_{0}^{\prime \prime}(x)^{2} \\
& {\left[+\frac{1}{2}\left(\sum_{i=1,3} A_{35}^{i}+\sum_{i=2,4} B_{35}^{i}\right)\left[\theta^{\prime \prime}(x)^{2}+\chi^{\prime \prime}(x)^{2}\right]+\frac{1}{2}\left(\sum_{i=1,3} A_{9}^{i}+\sum_{i=2,4} B_{35}^{i}\right) w_{0}^{\prime \prime}(x)^{2}-\sum_{i=1,3} A_{34}^{i} v_{0}^{\prime \prime}(x) \chi^{\prime \prime}(x)+\sum_{i=2,4} B_{34}^{i} w_{0}^{\prime \prime}(x) \chi^{\prime \prime}(x)\right]} \\
& M_{Z Y}=E\left[\begin{array}{l}
\quad \frac{1}{2}\left(\sum_{i=1,3} A_{41}^{i}+\sum_{i=2,4} B_{41}^{i}\right) \chi^{\prime}(x)^{2}-\frac{1}{2}\left(\sum_{i=1,3} A_{17}^{i}+\sum_{i=2,4} B_{17}^{i}\right) \theta^{\prime}(x) \chi^{\prime}(x)+\left(\sum_{i=1,3} A_{6}^{i}-\sum_{i=2,4} B_{6}^{i}\right)\left[\theta^{\prime \prime}(x)+u_{0}^{\prime}(x) \theta^{\prime \prime}(x)\right] \\
-\frac{1}{4} \sum_{i=1,3} A_{38}^{i}\left[v_{0}^{\prime \prime}(x) \zeta_{z}^{\prime}(x)+\chi^{\prime \prime}(x) \zeta_{z}^{\prime}(x)\right]+\left(\sum_{i=1,3} A_{7}^{i}+\sum_{i=2,4} B_{7}^{i}\right) v_{0}^{\prime \prime}(x) w_{0}^{\prime \prime}(x)+\sum_{i=1,3} A_{8}^{i} w_{0}^{\prime \prime}(x) \chi^{\prime \prime}(x)+\sum_{i=2,4} B_{8}^{i} v_{0}^{\prime \prime}(x) \chi^{\prime \prime}(x)
\end{array}\right], \\
& M_{Z \omega_{a}}=E\left[\left(\sum_{i=2,4} B_{7}^{i}-\sum_{i=1,3} A_{7}^{i}\right)\left[v_{0}^{\prime \prime}(x)+u_{0}^{\prime}(x) v_{0}^{\prime \prime}(x)\right]-\left(\sum_{i=1,3} A_{33}^{i}+\sum_{i=2,4} B_{28}^{i}\right) w_{0}^{\prime \prime}(x) \theta^{\prime \prime}(x)-\sum_{i=2,4} B_{8}^{i} \theta^{\prime \prime}(x) \chi^{\prime \prime}(x)-\sum_{i=2,4} B_{7}^{i} w_{0}^{\prime}(x) \chi^{\prime}(x)\right] \text {, } \\
& M_{\omega_{a} \omega_{d}}=E\left[\begin{array}{l}
-\sum_{i=1,3} A_{66}^{i}\left[\zeta_{z}^{\prime}(x)+u_{0}^{\prime}(x) \zeta_{z}^{\prime}(x)\right]+\frac{7}{6} \sum_{i=1,3} A_{62}^{i} w_{0}^{\prime \prime}(x) \zeta_{z}^{\prime}(x)+\sum_{i=2,4} B_{8}^{i} w_{0}^{\prime \prime}(x) \theta^{\prime \prime}(x) \\
+\sum_{i=1,3} A_{8}^{i}\left[w_{0}^{\prime \prime}(x)+u_{0}^{\prime}(x) w_{0}^{\prime \prime}(x)+v_{0}^{\prime \prime}(x) \theta^{\prime \prime}(x)\right]+\left(\sum_{i=2,4} B_{35}^{i}+\sum_{i=1,3} A_{35}^{i}\right) \theta^{\prime \prime}(x) \chi^{\prime \prime}(x)
\end{array}\right] \\
& M_{Z \omega_{d}}=E\left[\begin{array}{l}
-\sum_{i=2,4} B_{6}^{i}\left[u_{0}^{\prime}(x)+\frac{1}{2}\left(u_{0}^{\prime}(x)^{2}+v_{0}^{\prime}(x)^{2}+w_{0}^{\prime}(x)^{2}\right)\right]-\frac{1}{2} \sum_{i=2,4} B_{40}^{i} \theta_{0}^{\prime}(x)^{2}+\frac{1}{2}\left(\sum_{i=1,3} A_{53}^{i}-\sum_{i=2,4} B_{52}^{i}\right) \chi^{\prime}(x)^{2} \\
-\left(2 \sum_{i=1,3} A_{18}^{i}+\sum_{i=2,4} B_{57}^{i}\right) \theta_{0}^{\prime}(x) \chi^{\prime}(x)+\sum_{i=1,3} A_{60}^{i} v_{0}^{\prime \prime}(x) \zeta_{z}^{\prime}(x)-\left(\sum_{i=1,3} A_{7}^{i}+\sum_{i=2,4} B_{7}^{i}\right) w_{0}^{\prime}(x) \theta_{0}^{\prime}(x)-\frac{1}{2} \sum_{i=2,4} B_{8}^{i} v_{0}^{\prime \prime}(x)^{2} \\
-\frac{1}{2} \sum_{i=2,4} B_{34}^{i} \theta^{\prime \prime}(x)^{2}-\sum_{i=1,3} A_{34}^{i} v_{0}^{\prime \prime}(x) w_{0}^{\prime \prime}(x)-\left(\sum_{i=1,3} A_{33}^{i}+\sum_{i=2,4} B_{9}^{i}\right) w_{0}^{\prime \prime}(x) \chi_{0}^{\prime \prime}(x)
\end{array}\right],
\end{aligned}
$$




$$
\begin{aligned}
& M_{Y \omega_{a}}=E\left[\begin{array}{c}
\left(\sum_{i=2,4} B_{7}^{i}-\sum_{i=1,3} A_{7}^{i}\right)\left[w_{0}^{\prime \prime}(x)+u_{0}^{\prime}(x) w_{0}^{\prime \prime}(x)\right] \frac{1}{4} \sum_{i=1,3} A_{38}^{i}\left[\zeta_{z}^{\prime}(x)+u_{0}^{\prime}(x) \zeta_{z}^{\prime}(x)\right]-\sum_{i=1,3} A_{60}^{i} w_{0}^{\prime \prime}(x) \zeta_{z}^{\prime}(x) \\
+\sum_{i=2,4} B_{8}^{i}\left[\chi^{\prime \prime}(x)+u_{0}^{\prime}(x) \chi^{\prime \prime}(x)\right]-\left(\sum_{i=2,4} B_{33}^{i}+\sum_{i=1,3} A_{9}^{i}\right) v_{0}^{\prime \prime}(x) \theta_{0}^{\prime \prime}(x)-\sum_{i=1,3} A_{8}^{i} \theta_{0}^{\prime \prime}(x) \chi^{\prime \prime}(x)-\sum_{i=1,3} A_{2}^{i} v_{0}^{\prime}(x) \chi^{\prime}(x)
\end{array}\right], \\
& M_{Y \omega_{d}}=E\left[\begin{array}{l}
-\sum_{i=1,3} A_{6}^{i}\left[u_{0}^{\prime}(x)+\frac{1}{2}\left(u_{0}^{\prime}(x)^{2}+v_{0}^{\prime}(x)^{2}+w_{0}^{\prime}(x)^{2}\right)\right]-\frac{1}{2} \sum_{i=1,3} A_{40}^{i} \theta_{0}(x)^{2}-\frac{1}{2}\left(\sum_{i=1,3} A_{52}^{i}+\sum_{i=2,4} B_{53}^{i}\right) \chi^{\prime}(x)^{2} \\
+\left(2 \sum_{i=1,3} A_{18}^{i}+\sum_{i=2,4} B_{57}^{i}\right) \theta_{0}^{\prime}(x) \chi^{\prime}(x)+\sum_{i=2,4} B_{8}^{i}\left[\theta^{\prime \prime}(x)+u_{0}^{\prime}(x) \theta^{\prime \prime}(x)\right]-\frac{3}{7} \sum_{i=1,3} A_{60}^{i} \zeta_{z}^{\prime}(x)^{2}
\end{array}\right], \\
& Q_{\omega_{\zeta_{z}^{\prime}}}=G\left[\begin{array}{c}
\sum_{i=1,3} A_{F}^{i} \zeta_{z}(x)-2 \sum_{i=1,3} A_{37}^{i}\left[v_{0}^{\prime}(x) \theta^{\prime \prime}(x)+v_{0}^{\prime \prime}(x) \theta^{\prime}(x)\right]-\frac{4}{5} \sum_{i=1,3} A_{36}^{i}\left[\theta^{\prime}(x) \chi^{\prime \prime}(x)+\theta^{\prime \prime}(x) \chi^{\prime}(x)\right] \\
+\sum_{i=1,3} A_{8}^{i} w_{0}^{\prime}(x) \chi(x)-\sum_{i=1,3} A_{63}^{i} u_{0}^{\prime}(x) \zeta_{z}(x)
\end{array}\right], \\
& M_{s v}=G\left[\begin{array}{c}
-2 \sum_{i=1,3} A_{2}^{i}\left[u_{0}^{\prime}(x) \theta_{0}^{\prime}(x)+\chi^{\prime}(x)+v_{0}^{\prime}(x) w_{0}^{\prime \prime}(x)\right]+\left(\sum_{i=1,3} A_{65}^{i}-\sum_{i=2,4} B_{65}^{i}+\sum_{i=1,3} A_{66}^{i}+\sum_{i=2,4} B_{66}^{i}\right) \theta_{0}(x) \chi_{\mathbf{0}}^{\prime}(x) \\
+4 \sum_{i=1,3} A_{11}^{i} \theta_{0}^{\prime}(x) \chi_{0}(x)+\left(10 \sum_{i=2,4} B_{13}^{i}-10 \sum_{i=1,3} A_{13}^{i}-2 \sum_{i=1,3} A_{67}^{i}-\sum_{i=1,3} A_{78}^{i}-\sum_{i=2,4} B_{78}^{i}\right) \chi_{0}(x) \chi_{\mathbf{0}}^{\prime}(x) \\
\quad-\sum_{i=1,3} A_{3}^{i} w_{0}^{\prime \prime}(x) \chi_{0}^{\prime}(x)+\sum_{i=2,4} B_{3}^{i} v_{0}^{\prime \prime}(x) \chi_{0}^{\prime}(x)-\frac{4}{5} \sum_{i=1,3} A_{36}^{i} \chi_{0}^{\prime \prime}(x) \zeta_{z}(x)-4 \sum_{i=1,3} A_{37}^{i} v_{0}^{\prime \prime}(x) \zeta_{z}(x)
\end{array}\right], \\
& M_{s v \psi}=G\left[\begin{array}{c}
\sum_{i=2,4} B_{1}^{i}\left[2 w_{0}^{\prime}(x)+u_{0}^{\prime}(x) w_{0}^{\prime}(x)+v_{0}^{\prime}(x) \theta_{0}(x)\right]+\sum_{i=1,3} A_{1}^{i}\left[2 v_{0}^{\prime}(x)+u_{0}^{\prime}(x) v_{0}^{\prime}(x)+v_{0}^{\prime}(x) w_{0}^{\prime}(x)\right] \\
+\left(\sum_{i=1,3} A_{2}^{i}-\sum_{i=2,4} B_{2}^{i}\right) \theta_{0}^{\prime}(x)+2 \sum_{i=1,3} A_{2}^{i} u_{0}^{\prime}(x) \theta_{0}^{\prime}(x)+\left(\sum_{i=1,3} A_{19}^{i}+\sum_{i=2,4} B_{19}^{i}\right) \chi^{\prime}(x)+\sum_{i=1,3} A_{3}^{i} w_{0}(x) \theta_{0}^{\prime}(x) \\
-\sum_{i=1,3} A_{66}^{i} w_{0}^{\prime}(x) \chi_{0}(x)-\sum_{i=2,4} B_{66}^{i} v_{0}^{\prime}(x) \chi_{0}(x)-2\left(\sum_{i=1,3} A_{11}^{i}+\sum_{i=2,4} B_{11}^{i}\right) \theta_{0}^{\prime}(x) \chi_{0}(x)-\sum_{i=2,4} B_{3}^{i \prime} v_{0}(x) \theta_{0}^{\prime}(x) \\
-\left(\sum_{i=1,3} A_{66}^{i}+\sum_{i=2,4} B_{66}^{i}\right) \theta_{0}(x) \chi^{\prime}(x)+\left(\sum_{i=1,3} A_{94}^{i}+\sum_{i=2,4} B_{94}^{i}+\sum_{i=1,3} A_{78}^{i}+\sum_{i=2,4} B_{78}^{i}\right) \chi_{0}(x) \chi_{\mathbf{0}}^{\prime}(x)-\sum_{i=1,3} A_{37}^{i} v_{\mathbf{0}}^{\prime}(x) \zeta_{z}^{\prime}(x) \\
+\sum_{i=1,3} A_{2}^{i} v_{\mathbf{0}}^{\prime}(x) w_{\mathbf{0}}^{\prime \prime}(x)+\sum_{i=2,4} B_{2}^{i} v_{\mathbf{0}}^{\prime \prime}(x) w_{\mathbf{0}}^{\prime}(x)+2 \sum_{i=1,3} A_{37}^{i} v_{\mathbf{0}}^{\prime \prime}(x) \zeta_{z}(x)+\frac{4}{5} \sum_{i=1,3} A_{36}^{i}\left[\chi_{\mathbf{0}}^{\prime \prime}(x) \zeta_{z}(x)+\theta_{0}^{\prime \prime}(x) \zeta_{z}(x)\right]
\end{array}\right], \\
& M_{1}=G\left[\begin{array}{rl}
\left.\left(\sum_{i=1,3} A_{5}^{i}+\sum_{i=2,4} B_{5}^{i}\right) \theta_{0}(x) \theta_{0}^{\prime}(x)-\left(\sum_{i=1,3} A_{13}^{i}+\sum_{i=2,4} B_{13}^{i}\right) \theta_{0}(x) \chi_{0}^{\prime}(x)-3\left(\sum_{i=1,3} A_{51}^{i}+\sum_{i=2,4} B_{51}^{i}\right) \theta_{0}^{\prime}(x) \chi_{0}(x)\right] \\
\quad+\left(\sum_{i=2,4} B_{76}^{i}-\sum_{i=1,3} A_{76}^{i}\right) \chi_{0}(x) \chi_{0}^{\prime}(x)+\sum_{i=1,3} A_{5}^{i} v_{0}^{\prime}(x) v_{0}^{\prime \prime}(x)+\sum_{i=2,4} B_{5}^{i} w_{0}^{\prime}(x) w_{0}^{\prime \prime}(x) \\
+ & 2 \sum_{i=1,3} A_{37}^{i} w_{0}^{\prime}(x) \zeta_{z}(x)+\sum_{i=1,3} A_{36}^{i} \zeta_{z}(x) \zeta_{z}^{\prime}(x)+\sum_{i=1,3} A_{6}^{i}\left[v_{0}^{\prime}(x) w_{0}^{\prime \prime}(x)+v_{0}^{\prime \prime}(x) \chi_{0}^{\prime}(x)\right] \\
& +\left(\sum_{i=1,3} A_{7}^{i}+\sum_{i=2,4} B_{7}^{i}\right)\left[\theta_{0}^{\prime}(x) \theta_{0}^{\prime \prime}(x)+\chi_{0}^{\prime}(x) \chi_{0}^{\prime \prime}(x)\right]+\left(\sum_{i=1,3} A_{6}^{i}+\sum_{i=2,4} B_{6}^{i}\right) w_{0}^{\prime}(x) \chi_{0}^{\prime \prime}(x)
\end{array}\right]
\end{aligned}
$$




$$
\begin{aligned}
& M_{2}=G\left[\begin{array}{c}
\left(\sum_{i=1,3} A_{65}^{i}-\sum_{i=2,4} B_{65}^{i}\right)\left[\theta_{0}^{\prime}(x)+u_{0}^{\prime}(x) \theta_{0}^{\prime}(x)\right]+\sum_{i=2,4} B_{13}^{i} w_{0}^{\prime}(x) w_{0}^{\prime \prime}(x)+\sum_{i=2,4} B_{65}^{i} w_{0}^{\prime}(x) v_{0}^{\prime \prime}(x)+\sum_{i=2,4} B_{79}^{i} w_{0}^{\prime}(x) \chi_{0}^{\prime \prime}(x) \\
+\sum_{i=1,3} A_{13}^{i} v_{0}^{\prime}(x) v_{0}^{\prime \prime}(x)+\sum_{i=1,3} A_{65}^{i} v_{0}^{\prime}(x) w_{0}^{\prime \prime}(x)-\sum_{i=1,3} A_{73}^{i} v_{0}^{\prime}(x) \zeta_{z}(x)-\sum_{i=1,3} A_{79}^{i} v_{0}^{\prime}(x) \chi_{0}^{\prime \prime}(x)+\sum_{i=1,3} A_{84}^{i} w_{0}^{\prime}(x) \zeta_{z}(x) \\
+\sum_{i=1,3} A_{86}^{i} \zeta_{z}(x) \zeta_{z}^{\prime}(x)-\sum_{i=1,3} A_{88}^{i} \chi_{0}^{\prime \prime}(x) \zeta_{z}(x)+\left(\frac{1}{2} \sum_{i=1,3} A_{12}^{i}+\sum_{i=2,4} B_{79}^{i}\right) w_{0}^{\prime \prime}(x) \chi_{0}^{\prime}(x) \\
+\left(\sum_{i=1,3} A_{79}^{i}+\frac{1}{2} \sum_{i=2,4} B_{12}^{i}\right) v_{0}^{\prime \prime}(x) \chi_{0}^{\prime}(x)-\sum_{i=1,3} A_{39}^{i} \chi_{0}^{\prime}(x) \zeta_{z}^{\prime}(x)-\left(\sum_{i=1,3} A_{76}^{i}+\sum_{i=2,4} B_{76}^{i}\right) \theta_{0}^{\prime}(x) \chi_{0}(x) \\
+\left(\sum_{i=1,3} A_{7}^{i}-\sum_{i=1,3} A_{15}^{i}+\sum_{i=2,4} B_{7}^{i}+\sum_{i=2,4} B_{15}^{i}\right)\left[\theta_{0}^{\prime}(x) \theta_{0}(x)+\chi^{\prime}(x) \chi^{\prime \prime}(x)\right]-\left(\sum_{i=1,3} A_{97}^{i}+\sum_{i=2,4} B_{97}^{i}\right) \theta_{0}(x) \chi^{\prime}(x) \\
+\left(\sum_{i=1,3} A_{94}^{i}-\sum_{i=1,3} A_{98}^{i}+\sum_{i=2,4} B_{94}^{i}+\sum_{i=2,4} B_{98}^{i}\right) \chi_{0}(x) \chi_{0}^{\prime}(x)+\left(\sum_{i=1,3} A_{13}^{i}+\sum_{i=2,4} B_{13}^{i}\right) \theta_{0}(x) \theta_{0}^{\prime}(x)
\end{array}\right], \\
& {\left[\begin{array}{c}
-\left(\sum_{i=1,3} A_{67}^{i}+\sum_{i=2,4} B_{67}^{i}\right) \theta_{0}(x)+\left(10 \sum_{i=1,3} A_{14}^{i}-10 \sum_{i=2,4} B_{14}^{i}+\sum_{i=1,3} A_{67}^{i}-\sum_{i=2,4} B_{67}^{i}\right)\left[\theta_{0}^{\prime}(x)+u_{0}^{\prime}(x) \theta_{0}^{\prime}(x)\right] \\
-\left(\sum_{i=1,3} A_{78}^{i}+\sum_{i=2,4} B_{78}^{i}\right) \theta_{0}^{\prime}(x)+\left(\sum_{i=1,3} A_{78}^{i}-\sum_{i=2,4} B_{78}^{i}+\sum_{i=1,3} A_{94}^{i}-\sum_{i=2,4} B_{94}^{i}\right) \chi_{0}^{\prime}(x)+\left(\sum_{i=2,4} B_{76}^{i}-\sum_{i=1,3} A_{76}^{i}\right) \theta_{0}(x) \theta_{0}^{\prime}(x) \\
+\left(\sum_{i=1,3} A_{93}^{i}+\sum_{i=2,4} B_{93}^{i}+2 \sum_{i=1,3} A_{96}^{i}+2 \sum_{i=2,4} B_{96}^{i}+\sum_{i=1,3} A_{31}^{i}+\sum_{i=2,4} B_{31}^{i}\right) \chi_{0}(x) \chi_{0}^{\prime}(x)-\left(\sum_{i=1,3} A_{85}^{i}+\frac{7}{2} \sum_{i=1,3} A_{187}^{i}\right) \chi_{0}^{\prime}(x) \zeta_{z}^{\prime}(x) \\
-\left(\sum_{i=1,3} A_{94}^{i}-\sum_{i=1,3} A_{98}^{i}+\sum_{i=2,4} B_{94}^{i}+\sum_{i=2,4} B_{98}^{i}\right) \theta_{0}(x) \chi_{0}^{\prime}(x)+\frac{34}{91} \sum_{i=1,3} A_{83}^{i} \zeta_{z}(x) \zeta_{z}^{\prime}(x)+\left(\sum_{i=1,3} A_{83}^{i}+\sum_{i=1,3} A_{85}^{i}\right) w_{0}^{\prime \prime}(x) \zeta_{z}(x) \\
-\left(\sum_{i=1,3} A_{95}^{i}+\sum_{i=1,3} A_{100}^{i}+\sum_{i=2,4} B_{95}^{i}-\sum_{i=2,4} B_{100}^{i}\right) \theta_{0}^{\prime}(x) \chi_{0}(x)-\frac{21}{2} \sum_{i=1,3} A_{87}^{i}\left[\theta_{0}^{\prime \prime}(x) \zeta_{z}(x)-\chi_{0}^{\prime \prime}(x) \zeta_{z}(x)\right] \\
+\left(\sum_{i=2,4} B_{68}^{i}-\sum_{i=2,4} B_{76}^{i}\right) w_{0}^{\prime}(x) w_{0}^{\prime \prime}(x)+\left(\sum_{i=2,4} B_{68}^{i}-\sum_{i=2,4} B_{77}^{i}\right) w_{0}^{\prime}(x) \chi_{0}^{\prime \prime}(x)+\left(\sum_{i=1,3} A_{68}^{i}+\sum_{i=1,3} A_{76}^{i}\right) v_{0}^{\prime}(x) v_{0}^{\prime \prime}(x) \\
+\left(\sum_{i=1,3} A_{67}^{i}+\sum_{i=1,3} A_{77}^{i}\right) v_{0}^{\prime}(x) w_{0}^{\prime \prime}(x)-\left(\sum_{i=1,3} A_{72}^{i}+\sum_{i=1,3} A_{75}^{i}\right) v_{0}^{\prime}(x) \zeta_{z}^{\prime}(x)+\left(\sum_{i=1,3} A_{69}^{i}+\sum_{i=1,3} A_{77}^{i}\right) v_{0}^{\prime}(x) \chi_{0}^{\prime \prime}(x) \\
+\left(\sum_{i=1,3} A_{89}^{i}+\sum_{i=1,3} A_{77}^{i}+\sum_{i=2,4} B_{69}^{i}+10 \sum_{i=2,4} B_{15}^{i}\right) w_{0}^{\prime \prime}(x) \chi_{0}^{\prime}(x)+\left(\sum_{i=1,3} A_{90}^{i}+\sum_{i=2,4} B_{70}^{i}\right) v_{0}^{\prime \prime}(x) \chi_{0}^{\prime}(x) \\
+\left(\sum_{i=1,3} A_{69}^{i}+\sum_{i=2,4} B_{69}^{i}+\sum_{i=1,3} A_{95}^{i}+\sum_{i=2,4} B_{95}^{i}\right)\left[\theta_{0}^{\prime}(x) \theta_{0}^{\prime \prime}(x)+\chi_{0}^{\prime}(x) \chi_{0}^{\prime \prime}(x)\right]+\sum_{i=2,4} B_{78}^{i} w_{0}^{\prime}(x) v_{0}^{\prime \prime}(x)
\end{array}\right],}
\end{aligned}
$$




$$
\begin{aligned}
& -\left(\sum_{i=1,3} A_{92}^{i}+\sum_{i=2,4} B_{92}^{i}+\sum_{i=1,3} A_{76}^{i} \sum_{i=2,4} B_{76}^{i}+\right) \theta_{0}(x) \chi_{0}^{\prime}(x)+2\left(\sum_{i=1,3} A_{11}^{i}-\sum_{i=2,4} B_{11}^{i}\right)\left[\theta_{0}^{\prime}(x)+u_{0}^{\prime}(x) \theta_{0}^{\prime}(x)\right] \\
& +3 \sum_{i=2,4} B_{13}^{i} w_{0}^{\prime}(x) w_{0}^{\prime \prime}(x)+2 \sum_{i=2,4} B_{11}^{i} w_{0}^{\prime}(x) v_{0}^{\prime \prime}(x)+3 \sum_{i=2,4} B_{79}^{i}\left[w_{0}^{\prime}(x) \chi_{0}^{\prime \prime}(x)-v_{0}^{\prime}(x) \chi_{0}^{\prime \prime}(x)\right] \\
& +3 \sum_{i=1,3} A_{13}^{i} v_{0}^{\prime}(x) v_{0}^{\prime \prime}(x)+2 \sum_{i=1,3} A_{11}^{i} v_{0}^{\prime}(x) w_{0}^{\prime \prime}(x)+2 \sum_{i=1,3} A_{73}^{i}\left[3 v_{0}^{\prime \prime}(x) \zeta_{z}(x)-v_{0}^{\prime}(x) \zeta_{z}^{\prime \prime}(x)\right]+3 \sum_{i=1,3} A_{84}^{i} w_{0}^{\prime \prime}(x) \zeta_{z}(x) \\
& +6 \sum_{i=1,3} A_{88}^{i}\left[3 \chi_{0}^{\prime \prime} 0(x) \zeta_{z}(x)-\chi_{0}^{\prime}(x) \zeta_{z}(x)\right]+\left(\sum_{i=1,3} A_{12}^{i}+3 \sum_{i=1,3} A_{13}^{i}\right) w_{0}^{\prime \prime}(x) \chi_{0}^{\prime}(x)+\sum_{i=1,3} A_{62}^{i} \zeta_{z}(x) \zeta_{z}^{\prime}(x) \\
& +3\left(\sum_{i=1,3} A_{15}^{i}+\sum_{i=2,4} B_{15}^{i}\right)\left[\theta_{0}^{\prime}(x) \theta_{0}^{\prime \prime}(x)+\chi^{\prime}(x) \chi^{\prime \prime}(x)\right]+\left(3 \sum_{i=1,3} A_{13}^{i}+\sum_{i=2,4} B_{12}^{i}\right) v_{0}^{\prime \prime}(x) \chi_{0}^{\prime}(x) \\
& +\left(\sum_{i=1,3} A_{95}^{i}+\sum_{i=1,3} A_{100}^{i}+\sum_{i=2,4} B_{95}^{i}+\sum_{i=2,4} B_{100}^{i}\right) \chi_{0}(x) \chi_{0}^{\prime}(x)+\left(\sum_{i=2,4} B_{76}^{i}+\sum_{i=1,3} A_{76}^{i}\right) \theta_{0}(x) \theta_{0}^{\prime}(x) \\
& -\left(\sum_{i=1,3} A_{99}^{i}+\sum_{i=2,4} B_{99}^{i}\right) \theta_{0}^{\prime}(x) \chi_{0}(x)-2\left(\sum_{i=1,3} A_{11}^{i}+\sum_{i=2,4} B_{11}^{i}\right)\left[\theta_{0}^{\prime}(x)-\chi_{0}^{\prime}(x)\right] \\
& M_{\omega_{a}^{\prime} \omega_{\zeta z}}=G\left[2 \sum_{i=1,3} A_{36}^{i} \theta_{0}^{\prime}(x) \zeta_{z}^{\prime}(x)-\sum_{i=1,3} A_{37}^{i}\left[2 v_{0}^{\prime}(x)+w_{0}^{\prime}(x) \theta_{0}^{\prime}(x)\right]-5 \sum_{i=1,3} A_{82}^{i}\left[w_{0}^{\prime \prime}(x) \theta_{0}^{\prime}(x)+w_{0}^{\prime \prime}(x) \chi_{0}^{\prime}(x)\right]-6 \sum_{i=1,3} A_{88}^{i} w_{0}^{\prime}(x) \chi_{0}(x)\right] \text {, } \\
& M_{\omega_{d}^{\prime} \omega_{\zeta_{z}}}=G\left[\begin{array}{c}
\sum_{i=1,3} A_{38}^{i} v_{0}^{\prime}(x) \zeta_{z}^{\prime}(x)+2 \sum_{i=1,3} A_{36}^{i} \chi_{0}^{\prime}(x) \zeta_{z}^{\prime}(x)+\sum_{i=1,3} A_{39}^{i} \theta_{0}(x) \chi_{0}^{\prime}(x)-6 \sum_{i=1,3} A_{88}^{i} \theta_{0}^{\prime}(x) \chi_{0}(x) \\
\sum_{i=1,3} A_{82}^{i}\left[w_{0}^{\prime \prime}(x) \zeta_{z}^{\prime}(x)-\chi_{0}^{\prime \prime}(x) \zeta_{z}^{\prime}(x)+5 w_{0}^{\prime \prime}(x) \chi_{0}^{\prime}(x)\right]+\left(\frac{7}{2} \sum_{i=1,3} A_{87}^{i}-\sum_{i=1,3} A_{85}^{i}\right) \chi_{0}(x) \chi_{0}^{\prime}(x)
\end{array}\right], \\
& {\left[\left(\sum_{i=1,3} A_{69}^{i}+\sum_{i=1,3} A_{95}^{i}+\sum_{i=2,4} B_{69}^{i}+\sum_{i=2,4} B_{95}^{i}\right) \chi_{0}(x) \chi_{0}^{\prime}(x)+\left(\sum_{i=1,3} A_{7}^{i}+\sum_{i=2,4} B_{7}^{i}\right) \theta_{0}(x) \theta_{0}^{\prime}(x)\right.} \\
& M_{5}=G-\left(\sum_{i=1,3} A_{15}^{i}+\sum_{i=2,4} B_{15}^{i}\right)\left[\theta_{0}(x) \chi_{0}^{\prime}(x)+3 \theta_{0}^{\prime}(x) \chi_{0}(x)\right]+\sum_{i=1,3} A_{6}^{i} v_{0}^{\prime}(x) v_{0}^{\prime \prime}(x)+\sum_{i=1,3} A_{7}^{i} w_{0}^{\prime}(x) w_{0}^{\prime \prime}(x) \\
& +\sum_{i=2,4} B_{8}^{i}\left[w_{0}^{\prime}(x) \chi_{0}^{\prime \prime}(x)+w_{0}^{\prime \prime}(x) \chi_{0}^{\prime}(x)\right]+\sum_{i=1,3} A_{82}^{i}\left[\frac{5}{2} w_{0}^{\prime \prime}(x) \zeta_{z}(x)-\zeta_{z}(x) \zeta_{z}^{\prime}(x)\right] \\
& +\left(\sum_{i=1,3} A_{9}^{i}+\sum_{i=2,4} B_{9}^{i}\right)\left[\theta_{0}^{\prime}(x) \theta_{0}^{\prime \prime}(x)+\chi^{\prime}(x) \chi^{\prime \prime}(x)\right] \\
& \sum_{i=1,3} A_{7}^{i} w_{0}^{\prime}(x) w_{0}^{\prime \prime}(x)+\sum_{i=2,4} B_{8}^{i} w_{0}^{\prime}(x) \chi^{\prime \prime}(x)+\sum_{i=1,3} A_{6}^{i} v_{0}^{\prime}(x) v_{0}^{\prime \prime}(x)+\sum_{i=1,3} A_{3}^{i} v_{0}^{\prime}(x) \chi^{\prime \prime}(x) \\
& +\sum_{i=1,3} A_{82}^{i}\left[\frac{5}{2} w_{0}^{\prime \prime}(x) \zeta_{z}(x)-\zeta_{z}(x) \zeta_{z}^{\prime}(x)\right]+\sum_{i=2,4} B_{8}^{i}\left[w_{0}^{\prime \prime}(x) \chi_{0}^{\prime}(x)-v_{0}^{\prime \prime}(x) \chi_{0}^{\prime}(x)\right] \\
& M_{6}=G+\left(\sum_{i=1,3} A_{9}^{i}+\sum_{i=2,4} B_{9}^{i}\right)\left[\theta_{0}^{\prime}(x) \theta_{0}^{\prime \prime}(x)+\chi^{\prime}(x) \chi^{\prime \prime}(x)\right]+\left(\sum_{i=1,3} A_{69}^{i}+\sum_{i=1,3} A_{95}^{i}+\sum_{i=2,4} B_{69}^{i}+\sum_{i=2,4} B_{95}^{i}\right) \chi_{0}(x) \chi_{0}^{\prime}(x) \\
& +\left(\sum_{i=1,3} A_{7}^{i}+\sum_{i=2,4} B_{7}^{i}\right) \theta_{0}(x) \theta_{0}^{\prime}(x)-\left(\sum_{i=1,3} A_{15}^{i}+\sum_{i=2,4} B_{15}^{i}\right)\left[\theta_{0}(x) \chi_{0}^{\prime}(x)+3 \theta_{0}^{\prime}(x) \chi_{0}(x)\right] \\
& M_{7}=G\left[\frac{4}{5} \sum_{i=1,3} A_{36}^{i}\left[\zeta_{z}(x)+u_{0}^{\prime}(x) \zeta_{z}(x)\right]+2 \sum_{i=2,4} B_{8}^{i} w_{0}^{\prime}(x) \theta_{0}^{\prime \prime}(x)+\sum_{i=1,3} A_{3}^{i} v_{0}^{\prime}(x) \theta_{0}^{\prime \prime}(x)-\sum_{i=1,3} A_{12}^{i} w_{0}^{\prime}(x) \chi_{0}(x)+\sum_{i=2,4} B_{12}^{i} v_{0}^{\prime}(x) \chi_{0}(x)\right] \text {, } \\
& M_{8}=G\left[\begin{array}{c}
\frac{4}{5} \sum_{i=1,3} A_{36}^{i}\left[\zeta_{z}(x)+u_{0}^{\prime}(x) \zeta_{z}(x)\right]+\sum_{i=1,3} A_{3}^{i} v_{0}^{\prime}(x) \theta_{0}^{\prime \prime}(x)-\sum_{i=1,3} A_{12}^{i} w_{0}^{\prime}(x) \chi_{0}(x)+\sum_{i=2,4} B_{12}^{i} v_{0}^{\prime}(x) \chi_{0}(x) \\
+\sum_{i=2,4} B_{8}^{i}\left[v_{0}^{\prime \prime}(x) \theta_{0}^{\prime}(x)+2 w_{0}^{\prime}(x) \theta_{0}^{\prime \prime}(x)\right]-3\left(\sum_{i=1,3} A_{15}^{i}+\sum_{i=2,4} B_{15}^{i}\right) \theta_{0}^{\prime}(x) \chi_{0}(x)+\left(\sum_{i=1,3} A_{95}^{i}+\sum_{i=2,4} B_{95}^{i}\right) \theta_{0}(x) \chi_{0}^{\prime}(x)
\end{array}\right],
\end{aligned}
$$


Mathematical Problems in Engineering

27

in which $A_{i}(i=1, \ldots, 100)$ and $B_{i}(i=1, \ldots, 100)$ are parameters of beam cross section constants $\left(b, h, t_{i}\right) . B_{i}$ functions are calculated in the same way as $A_{i}$, except that $b$ is replaced by $h$.

$A_{F}^{i}=b t_{i}$

$A_{1}^{i}=\left(\frac{h}{2}-z_{0}\right) b t_{i}$,

$A_{2}^{i}=\left(\frac{h}{2}-z_{0}\right)^{2} b t_{i}$,

$A_{3}^{i}=\left(\frac{h}{2}-z_{0}\right)^{3} b t_{i}$,

$A_{4}^{i}=\left(\frac{h}{2}-z_{0}\right)^{4} b t_{i}$,

$A_{5}^{i}=\frac{b^{3} t_{i}}{12}$

$A_{6}^{i}=\left(\frac{h}{2}-z_{0}\right) \frac{b^{3} t_{i}}{12}$,

$A_{7}^{i}=\left(\frac{h}{2}-z_{0}\right)^{2} \frac{b^{3} t_{i}}{12}$,

$A_{8}^{i}=\left(\frac{h}{2}-z_{0}\right)^{3} \frac{b^{3} t_{i}}{12}$,

$A_{9}^{i}=\left(\frac{h}{2}-z_{0}\right)^{4} \frac{b^{3} t_{i}}{12}$,

$A_{10}^{i}=\frac{1}{2} \frac{b^{3} t_{i}}{(b+h)}$

$A_{11}^{i}=\frac{1}{2}\left(\frac{h}{2}-z_{0}\right) \frac{b^{3} t_{i}}{(b+h)}$,

$A_{12}^{i}=\left(\frac{h}{2}-z_{0}\right)^{2} \frac{b^{3} t_{i}}{2(b+h)}$,

$A_{13}^{i}=\frac{1}{20} \frac{h^{4} t_{i}}{(b+h)}$

$A_{14}^{i}=\left(\frac{h}{2}-z_{0}\right) \frac{b^{4} t_{i}}{20(b+h)}$,

$A_{15}^{i}=-\left(\frac{h}{2}-z_{0}\right)^{2} \frac{b^{4} t_{i}}{20(b+h)}$,

$A_{16}^{i}=\frac{b^{5} t_{i}}{60(b+h)}$
$A_{17}^{i}=\frac{17}{35}\left(\frac{h}{2}-z_{0}\right)^{2} \frac{b^{5} t_{i}}{(b+h)^{2}}+\left(\frac{3}{140}-\frac{17}{11 \cdot 2^{3}}\right) \frac{b^{8} t_{i}}{(b+h)^{3}}$

$+\left(\frac{h}{2}-z_{0}\right)^{4} b t_{i}-\left(\frac{h}{2}-z_{0}\right)^{2} \frac{23}{20} \frac{b^{3} t_{i}^{3}}{(b+h)^{2}}$

$-\left(\frac{h}{2}-z_{0}\right)^{2} \frac{b^{4} t_{i}}{20} b t_{i}-\frac{251}{15 \cdot 7 \cdot 2^{5}} \frac{b^{5} t_{i}^{3}}{(b+h)^{2}}$,

$A_{18}^{i}=\left(\frac{h}{2}-z_{0}\right)^{2} \frac{b^{5} t_{i}}{60(b+h)}$,

$A_{19}^{i}=\left(\frac{h}{2}-z_{0}\right)^{2} b t_{i}+\frac{23}{20} \frac{b^{3} t_{i}^{3}}{(b+h)^{2}}$,

$A_{20}^{i}=\frac{17}{35} \frac{b^{3} t_{i}}{(b+h)^{2}}$,

$A_{21}^{i}=\left(\frac{h}{2}-z_{0}\right)^{2} b t_{i}+\frac{1}{2} \frac{b^{4} t_{i}}{(b+h)}$,

$A_{22}^{i}=\left(\frac{h}{2}-z_{0}\right)\left[\left(\frac{h}{2}-z_{0}\right)^{2} b t_{i}+\frac{b^{3} t_{i}}{12}\right]$,

$A_{23}^{i}=\left(\frac{h}{2}-z_{0}\right)\left[\left(\frac{h}{2}-z_{0}\right)^{2} b t_{i}+\frac{23}{20} \frac{b^{3} t_{i}^{3}}{(b+h)^{2}}+\frac{17}{35} \frac{b^{5} t_{i}}{(b+h)^{2}}\right]$,

$A_{24}^{i}=\left(\frac{h}{2}-z_{0}\right)\left[\left(\frac{h}{2}-z_{0}\right)^{2} b t_{i}+\frac{23}{20} \frac{b^{3} t_{i}^{3}}{(b+h)^{2}}+\frac{1}{20} \frac{b^{4} t_{i}}{(b+h)}\right]$,

$A_{25}^{i}=\frac{1}{2}\left(\frac{h}{2}-z_{0}\right)^{2} \frac{b^{3} t_{i}}{(b+h)}+\frac{1}{120} \frac{b^{5} t_{i}}{(b+h)}$,

$A_{26}^{i}=\frac{1}{2}\left(\frac{h}{2}-z_{0}\right)^{2} \frac{b^{3} t_{i}}{(b+h)}+\frac{59}{280} \frac{b^{5} t_{i}^{3}}{(b+h)^{3}}-\frac{27}{10} \frac{b^{3} t_{i}}{(b+h)^{3}}$,

$A_{27}^{i}=\frac{1}{2}\left(\frac{h}{2}-z_{0}\right)^{2} \frac{b^{3} t_{i}}{(b+h)}+\frac{1}{140} \frac{b^{6} t_{i}}{(b+h)}$,

$A_{28}^{i}=\left(\frac{h}{2}-z_{0}\right)^{4} \frac{b^{5} t_{i}}{80}+\left(\frac{h}{2}-z_{0}\right)^{2} \frac{b^{3} t_{i}}{6}$,

$A_{29}^{i}=\left(\frac{h}{2}-z_{0}\right)^{4} b t_{i}+\frac{23}{20}\left(\frac{h}{2}-z_{0}\right)^{2} \frac{b^{3} t_{i}^{3}}{(b+h)^{2}}$

$+\frac{17}{35}\left(\frac{h}{2}-z_{0}\right)^{2} \frac{b^{5} t_{i}}{(b+h)^{2}}+\left(\frac{h}{2}-z_{0}\right)^{2} \frac{b^{3} t_{i}}{12}$

$+\frac{99}{560} \frac{b^{5} t_{i}^{3}}{(b+h)^{2}} \frac{71}{280} \frac{b^{7} t_{i}}{(b+h)^{2}}$

$A_{30}^{i}=\left(\frac{h}{2}-z_{0}\right)^{4} b t_{i}+\left(\frac{h}{2}-z_{0}\right)^{2} \frac{b^{3} t_{i}}{12}-\frac{1}{20}\left(\frac{h}{2}-z_{0}\right)^{2} \frac{b^{4} t_{i}}{b+h}$

$-\frac{1}{7 \cdot 2^{4}} \frac{b^{6} t_{i}}{b+h}$, 


$$
\begin{aligned}
& A_{31}^{i}=\frac{8557}{35 \cdot 11} \frac{b^{9} t_{i}}{(b+h)^{4}}, \\
& A_{49}^{i}=\frac{1}{840} \frac{h b^{6} t_{i}}{(b+h)^{2}}-\frac{3}{70} \frac{h b^{4} t_{i}^{3}}{(b+h)^{2}}, \\
& A_{32}^{i}=\frac{b^{5} t_{i}}{80}, \\
& A_{33}^{i}=\left(\frac{h}{2}-z_{0}\right)^{2} \frac{b^{5} t_{i}}{80}, \\
& A_{34}^{i}=\left(\frac{h}{2}-z_{0}\right)^{3} \frac{b^{5} t_{i}}{80} \\
& A_{35}^{i}=\left(\frac{h}{2}-z_{0}\right)^{4} \frac{b^{5} t_{i}}{80}, \\
& A_{36}^{i}=\frac{1}{15} h^{2} b t_{i} \text {, } \\
& A_{37}^{i}=\frac{1}{6} b h t_{i} \text {, } \\
& A_{38}^{i}=\frac{1}{30} h^{3} b t_{i}, \\
& A_{39}^{i}=\frac{1}{280} \frac{h b^{4} t_{i}}{(b+h)} \\
& A_{40}^{i}=\left(\frac{h}{2}-z_{0}\right)\left[\left(\frac{h}{2}-z_{0}\right)^{2} \frac{b^{3} t_{i}}{12}+\frac{b^{5} t_{i}}{80}\right] \text {, } \\
& A_{41}^{i}=\left(\frac{h}{2}-z_{0}\right)\left(-\frac{1}{140} \frac{b^{6} t_{i}}{(b+h)^{2}}+\frac{3}{10} \frac{b^{4} t_{i}^{3}}{(b+h)^{2}}\right) \text {, } \\
& A_{42}^{i}=\left(\frac{h}{2}-z_{0}\right)^{4} b t_{i}+\frac{36^{2} b^{5} t_{i}^{5}}{5(b+h)^{4}}\left(\frac{1}{5 \cdot 2^{4}}+\frac{1}{9 \cdot 2^{8}}+\frac{3}{7 \cdot 2^{5}}\right) \\
& +\frac{b^{9} t_{i}}{(b+h)^{4}}\left(\frac{1}{2^{4}}+\frac{3}{13 \cdot 2^{5}}-\frac{1}{70}+\frac{27}{11 \cdot 2^{3}}\right) \\
& +\frac{34}{35}\left(\frac{h}{2}-z_{0}\right)^{2} \frac{b^{5} t_{i}}{(b+h)^{4}}+\frac{b^{7} t_{i}^{3}}{(b+h)^{4}}\left(\frac{61}{140}+\frac{199}{3 \cdot 11 \cdot 2^{3}}\right), \\
& A_{50}^{i}=\left(\frac{h}{2}-z_{0}\right)^{2} \frac{2 h^{2} b t_{i}}{15}+\frac{1}{630} \frac{h^{2} b^{4} t_{i}}{(b+h)}, \\
& A_{51}^{i}=\frac{h^{4} b t_{i}}{24}+\frac{h b^{4} t_{i}}{140}, \\
& A_{52}^{i}=\left(\frac{h}{2}-z_{0}\right)\left[\left(\frac{h}{2}-z_{0}\right)^{2} \frac{b^{3} t_{i}}{12}-\frac{37}{630} \frac{b^{7} t_{i}}{(b+h)^{2}}+\frac{33}{560} \frac{b^{3} t_{i}^{3}}{(b+h)^{2}}\right]+ \\
& A_{53}^{i}=\left(\frac{h}{2}-z_{0}\right)^{2}\left(-\frac{1}{140} \frac{b^{4} t_{i}}{(b+h)^{2}}+\frac{3}{10} \frac{b^{3} t_{i}^{3}}{(b+h)^{2}}\right) \text {, } \\
& A_{54}^{i}=\left(\frac{h}{2}-z_{0}\right)^{4} b t_{i}+\frac{23}{20}\left(\frac{h}{2}-z_{0}\right)^{2} \frac{b^{3} t_{i}^{3}}{(b+h)^{2}}-\frac{1}{10} \frac{b^{4} t_{i}}{(b+h)}\left(\frac{h}{2}-z_{0}\right)^{2} \\
& +\frac{13}{40 \cdot 63} \frac{b^{7} t_{i}}{(b+h)^{2}} \\
& A_{55}^{i}=\left(\frac{h}{2}-z_{0}\right)^{2} \frac{b^{3} t_{i}}{12}-\frac{1}{7 \cdot 2^{4}} \frac{b^{7} t_{i}}{(b+h)}, \\
& A_{56}^{i}=\left(\frac{h}{2}-z_{0}\right)^{2}\left[\left(\frac{h}{2}-z_{0}\right)^{2} \frac{b^{3} t_{i}}{12}+\frac{1}{7.2^{4}} \frac{b^{6} t_{i}}{b+h}\right] \text {, } \\
& A_{57}^{i}=\left(\frac{h}{2}-z_{0}\right)\left[\left(\frac{h}{2}-z_{0}\right)^{2} \frac{b^{3} t_{i}}{12}+\mid \frac{1}{7.2^{4}} \frac{b^{6} t_{i}}{b+h}\right] \text {, } \\
& A_{58}^{i}=\frac{h b^{5} t_{i}}{420}, \\
& A_{59}^{i}=\frac{h^{2} b^{5} t_{i}}{840} \\
& A_{60}^{i}=\frac{b^{3} h^{3} t_{i}}{240}, \\
& A_{61}^{i}=-\frac{89 b^{3} h^{2} t_{i}}{420(b+h)} \\
& A_{62}^{i}=\frac{b^{3} h^{4} t_{i}}{840}, \\
& A_{43}^{i}=\left(\frac{h}{2}-z_{0}\right)^{2}\left[\left(\frac{h}{2}-z_{0}\right)^{2} b t_{i}+\frac{23}{20} \frac{b^{3} t_{i}^{3}}{(b+h)^{2}}+\frac{17}{35} \frac{b^{3} t_{i}}{(b+h)^{2}}\right] \text {, } \\
& A_{63}^{i}=\frac{h^{2} t_{i}}{3 b}, \\
& A_{44}^{i}=\frac{b^{7} t_{i}}{(b+h)^{2}}\left(\frac{1}{12}+\frac{9}{7 \cdot 2^{4}}+\frac{1}{9 \cdot 2^{4}}-\frac{3}{20}\right)+\left(\frac{h}{2}-z_{0}\right)^{3} \frac{b^{3} t_{i}}{12} \\
& +\frac{b^{5} t_{i}^{3}}{(b+h)^{2}}\left(\frac{3}{7 \cdot 2^{4}}+\frac{3}{20}\right) \\
& A_{45}^{i}=\frac{b^{3} h^{2} t_{i}}{210}, \\
& A_{46}^{i}=\frac{b h^{3} t_{i}}{12}+\frac{b^{3} h^{2} t_{i}}{12}, \\
& A_{47}^{i}=\frac{949}{140 \cdot 9 \cdot 11} \frac{h^{2} b^{5} t_{i}}{(b+h)^{2}}+\left(\frac{h}{2}-z_{0}\right)^{2} \frac{2 h^{2} b t_{i}}{15}+\frac{h^{2} b^{3} t_{i}^{3}}{140 \cdot 3}, \\
& A_{48}^{i}=\left(\frac{h}{2}\right)^{3} \frac{b t_{i}}{2}+\frac{b h^{3} t_{i}}{48}+\frac{127}{1260} \frac{h b^{5} t_{i}}{(b+h)^{2}}-\frac{31}{280} \frac{h b^{3} t_{i}^{3}}{(b+h)^{2}}, \\
& A_{64}^{i}=-\frac{1}{2} \frac{b^{2} t_{i}}{(b+h)}, \\
& A_{65}^{i}=\frac{1}{2}\left(\frac{h}{2}-z_{0}\right) \frac{b^{2} t_{i}}{(b+h)}, \\
& A_{66}^{i}=-\frac{1}{2}\left(\frac{h}{2}-z_{0}\right) \frac{b^{2} t_{i}}{(b+h)}, \\
& A_{67}^{i}=\frac{1}{36}\left(\frac{h}{2}-z_{0}\right) \frac{b^{3} t_{i}^{3}}{(b+h)} \\
& A_{68}^{i}=\frac{13}{10} \frac{b^{3} t_{i}^{3}}{(b+h)^{2}}, \\
& A_{69}^{i}=\frac{13}{10}\left(\frac{h}{2}-z_{0}\right) \frac{b^{3} t_{i}^{3}}{(b+h)^{2}}, \\
& A_{70}^{i}=\frac{13}{10}\left(\frac{h}{2}-z_{0}\right)^{2} \frac{b^{3} t_{i}^{3}}{(b+h)^{2}},
\end{aligned}
$$




$$
\begin{aligned}
& A_{71}^{i}=\frac{4 b h t_{i}}{b+h} \\
& A_{72}^{i}=\frac{3}{5} \frac{h b^{2} t_{i}^{3}}{(b+h)^{2}}, \\
& A_{73}^{i}=\frac{1}{10} \frac{h b^{3} t_{i}}{(b+h)}, \\
& A_{74}^{i}=\frac{1}{5} \frac{h b^{2} t_{i}}{(b+h)^{2}}, \\
& A_{75}^{i}=\frac{38}{35} \frac{h b^{5} t_{i}}{(b+h)^{2}}, \\
& A_{85}^{i}=\frac{1}{70} \frac{h^{2} b^{4} t_{i}}{(b+h)^{2}}, \\
& A_{76}^{i}=\frac{1}{140} \frac{b^{6} t_{i}}{(b+h)^{2}}, \\
& A_{83}^{i}=\frac{13}{5} \frac{h^{2} b^{2} t_{i}}{(b+h)}, \\
& A_{77}^{i}=\frac{h^{2} b t_{i}^{3}}{(b+h)^{2}}, \\
& A_{78}^{i}=\frac{h^{2} b^{2} t_{i}}{(b+h}, \\
& \left.A_{78}^{i}=\frac{\left(\frac{h}{2}-z_{0}\right.}{20}\right) \frac{b^{6} t_{i}}{(b+h)^{2}},
\end{aligned}
$$

$$
\begin{aligned}
& A_{87}^{i}=-\frac{3}{35} \frac{h^{2} b^{2} t_{i}}{(b+h)^{2}}, \\
& A_{88}^{i}=-\frac{1}{60} \frac{h^{2} b^{3} t_{i}}{(b+h)} \\
& A_{89}^{i}=-\frac{34}{35}\left(\frac{h}{2}-z_{0}\right)^{2} \frac{h^{2} b t_{i}^{3}}{(b+h)^{2}} \\
& A_{90}^{i}=\left(\frac{h}{2}-z_{0}\right) \frac{9 b^{4} t_{i}^{3}}{20(b+h)}, \\
& A_{91}^{i}=\frac{16 b t_{i}^{3}}{(b+h)^{2}}, \\
& A_{92}^{i}=\left(\frac{h}{2}-z_{0}\right)^{2} \frac{16 b t_{i}^{3}}{(b+h)^{2}}, \\
& A_{93}^{i}=\frac{27 \cdot 438}{245} \frac{h^{3} t_{i}^{5}}{(b+h)^{2}}, \\
& A_{94}^{i}=\left(\frac{h}{2}-z_{0}\right) \frac{6 b^{2} t_{i}^{3}}{(b+h)^{2}}, \\
& A_{95}^{i}=\left(\frac{h}{2}-z_{0}\right)^{2} \frac{b^{6} t_{i}}{140(b+h)^{2}}, \\
& A_{96}^{i}=\frac{53 \cdot 6}{35} \frac{b^{6} t_{i}^{3}}{(b+h)^{4}}, \\
& A_{97}^{i}=\frac{17}{35} \frac{b^{5} t_{i}^{3}}{(b+h)^{2}} \\
& A_{98}^{i}=\frac{5}{3} \frac{b^{7} t_{i}}{(b+h)^{3}}, \\
& A_{99}^{i}=\frac{33.9}{140} \frac{b^{5} t_{i}}{(b+h)^{2}}, \\
& A_{100}^{i}=\frac{251}{140} \frac{b^{7} t_{i}}{(b+h)^{3}} .
\end{aligned}
$$




\section{B. Algebraic System}

$$
\begin{aligned}
& P_{1}=-\sum_{i=4} F_{i} \\
& P_{2}=-\frac{8 \pi}{3 L^{2}} \sum_{i=1,3} A_{37}^{i} \\
& P_{3}=\frac{3 \pi^{4}}{4 L^{4}}\left(\sum_{i=1,3} A_{2}^{i}+\sum_{i=2,4} B_{5}^{i}\right)-\frac{3 \pi^{2}}{4 L^{2}} \sum_{i=4} F_{i} \\
& P_{4}=\frac{\pi^{2}}{L^{2}}\left(\sum_{i=1,3} A_{5}^{i}+\sum_{i=2,4} B_{2}^{i}\right) \text {, } \\
& P_{5}=-\frac{\pi^{2}}{L^{2}} \sum_{i=2,4} A_{6}^{i} \\
& P_{6}=\frac{4 \pi}{3 L^{2}}\left(4 \sum_{i=2,4} B_{2}^{i}+4 \sum_{i=1,3} A_{5}^{i}+\sum_{i=1,3} A_{2}^{i}+\sum_{i=2,4} B_{5}^{i}\right)-\frac{8 \pi^{3}}{3 L^{4}}\left(\sum_{i=1,3} A_{7}^{i}-\sum_{i=2,4} B_{7}^{i}-\sum_{i=1,3} A_{6}^{i}+\sum_{i=2,4} B_{6}^{i}\right), \\
& P_{7}=\frac{4 \pi}{8 L^{2}}\left(\sum_{i=1,3} A_{2}^{i}-\sum_{i=2,4} B_{5}^{i}-4 \sum_{i=1,3} A_{11}^{i}-4 \sum_{i=2,4} B_{2}^{i}\right) \text {, } \\
& P_{8}=\frac{\pi^{4}}{8 L^{4}}\left(\sum_{i=1,3} A_{2}^{i}+\sum_{i=2,4} B_{5}^{i}\right)-\frac{3 \pi^{6}}{8 L^{6}}\left(2 \sum_{i=1,3} A_{4}^{i}+2 \sum_{i=2,4} B_{32}^{i}+\sum_{i=1,3} A_{7}^{i}+\sum_{i=2,4} B_{7}^{i}\right), \\
& P_{9}=\frac{\pi^{2}}{L^{2}}\left(\sum_{i=1,3} A_{2}^{i}+\sum_{i=2,4} B_{5}^{i}\right) \text {, } \\
& P_{10}=\frac{\pi^{2}}{L^{2}} \sum_{i=2,4} B_{6}^{i} \text {, } \\
& P_{11}=-\frac{20 \pi}{3 L^{2}} \sum_{i=1,3} A_{11}^{i}, \\
& P_{12}=\frac{\pi^{4}}{8 L^{4}}\left(\sum_{i=1,3} A_{5}^{i}+\sum_{i=2,4} B_{2}^{i}\right)+\frac{3 \pi^{6}}{8 L^{6}}\left(\sum_{i=1,3} A_{7}^{i}+\sum_{i=2,4} B_{7}^{i}\right), \\
& P_{13}=\frac{E \pi^{2}}{L^{2}}\left(\sum_{i=1,3} A_{5}^{i}-\sum_{i=2,4} B_{7}^{i}\right), \\
& P_{14}=G \sum_{i=1,3} A_{2}^{i} \\
& P_{15}=\frac{4 E \pi}{3 L^{2}}\left(\sum_{i=1,3} A_{2}^{i}+\sum_{i=2,4} B_{5}^{i}\right)+\frac{8 E \pi^{3}}{3 L^{4}}\left(\sum_{i=2,4} B_{6}^{i}-\sum_{i=1,3} A_{6}^{i}\right)-\frac{8 G \pi}{3 L^{2}} \sum_{i=1,3} A_{2}^{i},
\end{aligned}
$$




$$
\begin{aligned}
& P_{16}=\frac{8 E \pi^{3}}{3 L^{4}}\left(\sum_{i=2,4} B_{8}^{i}-\sum_{i=1,3} A_{8}^{i}\right)+\frac{4 G \pi}{3 L^{2}} \sum_{i=1,3} A_{3}^{i}, \\
& P_{17}=\frac{8 E \pi^{3}}{3 L^{4}}\left(\sum_{i=2,4} B_{7}^{i}-\sum_{i=1,3} A_{7}^{i}\right) \text {, } \\
& P_{18}=\frac{3 E \pi^{2}}{3 L^{2}}\left(\sum_{i=1,3} A_{2}^{i}+\sum_{i=1,3} A_{5}^{i}+\sum_{i=2,4} B_{2}^{i}+\sum_{i=2,4} B_{5}^{i}\right)+\frac{2 E \pi}{3 L^{2}}\left(\sum_{i=1,3} A_{7}^{i}+\sum_{i=2,4} B_{5}^{i}+\sum_{i=2,4} A_{4}^{i}+\sum_{i=2,4} B_{32}^{i}\right) \\
& +\frac{E \pi^{4}}{8 L^{4}}\left(\sum_{i=1,3} A_{7}^{i}+\sum_{i=2,4} B_{7}^{i}\right)+\frac{3 E \pi^{6}}{8 L^{6}}\left(\sum_{i=1,3} A_{9}^{i}+\sum_{i=2,4} B_{33}^{i}\right)-\frac{G \pi^{2}}{2 L^{2}} \sum_{i=2,4} B_{5}^{i}+\frac{G \pi^{4}}{2 L^{4}} \sum_{i=1,3} A_{7}^{i}, \\
& P_{19}=\frac{3 E \pi^{2}}{8 L^{2}}\left(\sum_{i=1,3} A_{2}^{i}+\sum_{i=2,4} B_{21}^{i}\right)-\frac{E \pi^{4}}{4 L^{4}}\left(\sum_{i=1,3} A_{4}^{i}-\sum_{i=1,3} A_{15}^{i}+\sum_{i=2,4} B_{55}^{i}\right) \text {, } \\
& P_{20}=-2 G \sum_{i=2,4} B_{1}^{i} \\
& P_{21}=-2 G \sum_{i=1,3} A_{1}^{i} \text {, } \\
& P_{22}=-G\left(\sum_{i=1,3} A_{1}^{i}-\sum_{i=2,4} B_{2}^{i}\right) \text {, } \\
& P_{23}=-\frac{E \pi^{2}}{L^{2}}\left(\sum_{i=1,3} A_{7}^{i}+\sum_{i=2,4} B_{7}^{i}\right)-G\left(\sum_{i=1,3} A_{19}^{i}-\sum_{i=2,4} B_{19}^{i}\right) \text {, } \\
& P_{24}=\frac{4 G \pi}{3 L^{2}}\left(\sum_{i=1,3} A_{2}^{i}+\sum_{i=2,4} B_{2}^{i}\right) \\
& P_{25}=-\frac{16 E \pi}{3 L^{2}} \sum_{i=1,3} A_{6}^{i}+\frac{8 E \pi^{3}}{4 L^{4}}\left(\sum_{i=2,4} B_{8}^{i}-\sum_{i=1,3} A_{8}^{i}\right)-\frac{4 G}{3 \pi} \sum_{i=1,3} A_{1}^{i}+\frac{4 G \pi}{3 L^{2}} \sum_{i=1,3} A_{3}^{i}, \\
& P_{26}=\frac{4 E \pi}{3 L^{2}} \sum_{i=1,3} A_{14}^{i}+\frac{4 G}{3 \pi} \sum_{i=1,3} A_{66}^{i} \\
& P_{27}=-\frac{4 E \pi}{3 L^{2}} \sum_{i=1,3} A_{11}^{i} w_{0}(x)^{2} \text {, } \\
& P_{28}=\frac{3 E \pi^{2}}{8 L^{2}}\left(\sum_{i=1,3} A_{21}^{i}+\sum_{i=2,4} B_{21}^{i}\right)+\frac{E \pi^{4}}{4 L^{4}}\left(\sum_{i=1,3} A_{4}^{i}-2 \sum_{i=2,4} B_{7}^{i}-\sum_{i=1,3} A_{15}^{i}+\sum_{i=2,4} B_{2,4}^{i}\right)+\frac{G \pi^{2}}{L^{2}} \sum_{i=2,4} B_{13}^{i}, \\
& P_{29}=-\frac{3 E \pi^{2}}{8 L^{2}}\left(\sum_{i=1,3} A_{19}^{i}+\sum_{i=2,4} B_{19}^{i}+\sum_{i=1,3} A_{20}^{i}+\sum_{i=2,4} B_{20}^{i}\right)+\frac{3 E \pi^{6}}{8 L^{6}}\left(\sum_{i=1,3} A_{9}^{i}+\sum_{i=2,4} B_{33}^{i}\right) \frac{E \pi^{4}}{8 L^{4}}\left(\sum_{i=1,3} A_{7}^{i}+\sum_{i=2,4} B_{7}^{i}\right. \\
& \left.+2 \sum_{i=1,3} A_{43}^{i}+2 \sum_{i=2,4} B_{44}^{i}\right)-\frac{G \pi^{2}}{2 L^{2}}\left(\sum_{i=2,4} B_{68}^{i}+\sum_{i=2,4} B_{76}^{i}\right)+\frac{3 G \pi^{4}}{4 L^{4}} \sum_{i=1,3} A_{7}^{i} \\
& P_{30}=\frac{G L^{2}}{\pi^{2}} \sum_{i=1,3} A_{F}^{i} .
\end{aligned}
$$




\section{Data Availability}

All data and models generated or used during the study are included within the article.

\section{Conflicts of Interest}

The authors declare that they have no conflicts of interest.

\section{References}

[1] Vlasov, "Thin-walled elastic beams," Israel for Program for Scientific Translation, Jerusalem, Israel, 1961.

[2] M. M. Correia, F. Nunes, J. R. Correia, and N. Silvestre, "Buckling behavior and failure of hybrid fiber-reinforced polymer pultruded short columns," Journal of Composites for Construction, vol. 17, no. 4, pp. 463-475, 2013.

[3] L. Silvestre, V. P. Berardi, A. Giordano, and S. Spadea, "Local buckling behavior of FRP thin-walled beams: a mechanical model," Composite Structures, vol. 98, pp. 111-120, 2013.

[4] L. Spadea, V. P. Berardi, A. Giordano, and S. Spadea, "Buckling failure modes of FRP thin-walled beams," Composites Part B: Engineering, vol. 47, pp. 357-364, 2013.

[5] L. Spadea, V. P. Berardi, A. Giordano, and S. Spadea, "Macroscale analysis of local and global buckling behavior of T and C composite sections," Mechanics Research Communications, vol. 58, pp. 105-111, 2014.

[6] L. Spadea, V. P. Berardi, A. Giordano, and S. Spadea, "Prebuckling imperfection sensitivity of pultruded FRP profiles," Composites Part B: Engineering, vol. 72, pp. 206-212, 2015.

[7] E. Spadea, "Analysis of shear lag in box beams by the principle of minimum potential energy," Quarterly of Applied Mathematics, vol. 4, no. 3, pp. 268-278, 1946.

[8] Q. Z. Luo, Y. M. Wu, Q. S. Li, J. Tang, and G. D. Liu, "A finite segment model for shear lag analysis," Engineering Structures, vol. 26, no. 14, pp. 2113-2124, 2004.

[9] Q. Z. Liu, Q. S. Li, and J. Tang, "Shear lag in box girder bridges tang. Shear lag in box girder bridges," Journal of Bridge Engineering, vol. 7, no. 5, pp. 308-313, 2002.

[10] Y. Tang, S. Liu, Y. Zhu, and Y. Lai, "Matrix analysis of shear lag and shear deformation in thin-walled box beams," Journal of Engineering Mechanics, vol. 129, no. 8, pp. 944-950, 2003.

[11] S.-J. Lai, "Finite beam element considering shear-lag effect in box girder," Journal of Engineering Mechanics, vol. 136, no. 9, pp. 1115-1122, 2010.

[12] Y. H. Zhang, "Improved finite-segment method for analyzing shear lag effect in thin-walled box girders," Journal of Structural Engineering, vol. 138, no. 10, pp. 1279-1284, 2010.

[13] Z. Lin and J. Zhao, "Least-work solutions of flange normal stresses in thin-walled flexural members with high-order polynomial," Engineering Structures, vol. 33, no. 10, pp. 2754-2761, 2011.

[14] Z. Lin and J. Zhao, "Modeling inelastic shear lag in steel box beams," Engineering Structures, vol. 41, pp. 90-97, 2012.

[15] C. Lertsima, T. Chaisomphob, and E. Yamaguchi, "Stress concentration due to shear lag in simply supported box girders," Engineering Structures, vol. 26, no. 8, pp. 1093-1101, 2004.

[16] J. Sa-nguanmanasak, T. Chaisomphob, and E. Yamaguchi, "Stress concentration due to shear lag in continuous box girders," Engineering Structures, vol. 29, no. 7, pp. 1414-1421, 2007.
[17] Y. H. Zhang and L. X. Lin, "Shear lag analysis of thin-walled box girder based on a new generalized," Journal of Structural Engineering, vol. 64, pp. 73-83, 2014.

[18] J. Chen, S.-L. Shen, Z.-Y. Yin, and S. Horpibulsuk, "Closedform solution for shear lag with derived flange deformation function," Journal of Constructional Steel Research, vol. 102, pp. 104-110, 2014

[19] X. Horpibulsuk, S. Wan, Y. L. Mo, K. Shen, T. Zhou, and Y. Nian, "improved method for analyzing shear lag in thinwalled box-section beam with arbitrary width of cantilever flange," Thin-Walled Structures, vol. 140, pp. 222-235, 2019.

[20] X. Zhou, Q. Yang, and S.-s. Law, "A shear locking-free spatial beam element with general thin-walled closed cross-section," Engineering Structures, vol. 58, pp. 12-24, 2014.

[21] J. H. Law and Y. Y. Kim, "One-dimensional analysis of thinwalled closed beams having general cross-sections," International Journal for Numerical Methods in Engineering, vol. 49, no. 5, pp. 653-668, 2000.

[22] X. Xu and H. Ye, "Distortion analysis of thin-walled box girder considering shear deformation," Chinese Journal of Computational Mechanics, vol. 30, no. 6, pp. 860-866, 2013.

[23] A. G. Razaqpur and H. G. Li, "Refined analysis of curved thinwalled multicell box girders," Computers \& Structures, vol. 53, no. 1, pp. 131-142, 1994.

[24] I. S. Choi, G.-W. Jang, S. Choi, D. Shin, and Y. Y. Kim, "order analysis of thin-walled beams with axially varying quadrilateral cross sections," Computers \& Structures, vol. 179, pp. 127-139, 2017.

[25] M. Tan and W. M. Cheng, "Non-linear lateral buckling analysis of unequal thickness thin-walled box beam under an eccentric load," Thin-Walled Structures, vol. 139, pp. 77-90, 2019.

[26] A. Genoese, A. Genoese, A. Bilotta, and G. Garcea, "A generalized model for heterogeneous and anisotropic beams including section distortions," Thin-Walled Structures, vol. 74, pp. $85-103$.

[27] S. Garcea and W. Pietraszkiewicz, "Intrinsic equations for non-linear deformation and stability of thin elastic shells," International Journal of Solids and Structures, vol. 41, no. 1112, pp. 3275-3292, 2004.

[28] T. P. Vo and J. Lee, "Geometrically nonlinear theory of thinwalled composite box beams using shear-deformable beam theory," International Journal of Mechanical Sciences, vol. 52, no. 1, pp. 65-74, 2010.

[29] S. Miranda and A. Gutiérrez, "A generalized beam theory with shear deformation,” Thin-Walled Structures, vol. 67, pp. 88100, 2013.

[30] C. Zhang and S. Wang, "Structure mechanical modeling of thin-walled closed-section composite beams, Part 1: singlecell cross section," Composite Structures, vol. 113, pp. 12-22, 2014.

[31] A. Sahraei, L. Wu, and M. Mohareb, "Finite element formulation for lateral torsional buckling analysis of shear deformable mono-symmetric thin-walled members," ThinWalled Structures, vol. 89, pp. 212-226, 2015.

[32] F. Cambronero-Barrientos, J. Díaz-del-Valle, and J.-A. Martínez-Martínez, "Beam element for thin-walled beams with torsion, distortion, and shear lag," Engineering Structures, vol. 143, pp. 571-588, 2017.

[33] A. Martínez-Martínez, S. A. Meftah, F. Mohriau, and E. M. Daya, "Lateral buckling of box beam elements under combined axial and bending loads," Journal of Constructional Steel Research, vol. 116, pp. 141-155, 2016. 